\title{
Pseudocompact $\mathrm{C}^{*}$-Algebras
}

Stephen Raymond Hardy

Harvard, Massachusetts

B.A., Mathematics and Computer Science, McDaniel College, 2010

A Dissertation Presented to the Graduate Faculty of the University of Virginia in Candidacy for the Degree of Doctor of Philosophy

Department of Mathematics

University of Virginia

May, 2016 


\begin{abstract}
Finite dimensional $\mathrm{C}^{*}$-algebras are just finite direct sums of matrix algebras, and historically limits of finite dimensional $\mathrm{C}^{*}$-algebras, such as the algebra of compact operators, UHF algebras, and AF algebras have yielded nice classification results. We study the classes of pseudocompact and pseudomatricial $\mathrm{C}^{*}$-algebras, which are the logical limits of finite-dimensional $\mathrm{C}^{*}$-algebras and matrix algebras, respectively, using the continuous model theory for metric structures. To better understand ultraproducts, we discuss when elements in ultraproducts have nice representative sequences, study the relationships between the $\mathrm{C}^{*}$-algebras $\mathcal{K}\left(\mathcal{H}^{\mathcal{U}}\right), \mathcal{K}(\mathcal{H})^{\mathcal{U}}, \mathcal{B}(\mathcal{H})^{\mathcal{U}}$, and $\mathcal{B}\left(\mathcal{H}^{\mathcal{U}}\right)$, as well the relationship between the spectrum of an element $\left(x_{i}\right)_{\mathcal{U}}$ in an ultraproduct and the spectra of the $x_{i}$. We introduce a notion of an ultralimit of sets, give some useful containments, and a nice way to calculate spectra for normal elements with a normal representative sequence. We study how Lin's Theorem is related to finding normal representative sequences for normal elements in an ultraproduct. We show that all the algebras of compact operators on infinite-dimensional Hilbert spaces are elementarily equivalent. We summarize work of Henson/Moore and Eagle/Vignati classifying the commutative pseudocompact $\mathrm{C}^{*}$-algebras and give an explicit axiomatization of this class. We show that the unitization of the compact operators is not pseudocompact, UHF algebras are never pseudocompact. We show that the AF algebras and pseudocompacts are distinct. We show that direct sums, matrix amplifications, corners and centers of pseudocompact $\mathrm{C}^{*}$-algebras are pseudocompact, but subalgebras and quotients of pseudocompact $\mathrm{C}^{*}$-algebras need not be pseudocompact. We show pseudocompact $\mathrm{C}^{*}$-algebras have trivial $K_{1}$ groups. We show that in pseudocompact $\mathrm{C}^{*}$-algebras every non-zero projection dominates a minimal projection, and modulo minimal projections every projection can be split into approximate fractions. We show that pseudocompact $\mathrm{C}^{*}$-algebras have the Dixmier property. We show that the pseudomatricial $\mathrm{C}^{*}$-algebras are the pseudocompact $\mathrm{C}^{*}$-algebras with trivial centers and a unique tracial state. We show there are uncountably many $*$-isomorphism classes of separable pseudomatricial $\mathrm{C}^{*}$-algebras. We show that all projections in a pseudomatricial $\mathrm{C}^{*}$-algebra are comparable, that all notions of equivalence coincide, and pseudomatricial $\mathrm{C}^{*}$-algebras have strict comparison of projections. We show that infinite-dimensional pseudomatricial $\mathrm{C}^{*}$-algebras are never simple. We give some idea of what the $K_{0}$ groups of pseudomatricial $\mathrm{C}^{*}$ algebras look like, and show that separable infinite-dimensional $\mathrm{C}^{*}$-algebras can have many Archimedean classes of projections. We also show that in pseudomatricial $\mathrm{C}^{*}$ algebras, an element is a self-commutator if and only if it is self-adjoint and has trace zero. We conclude with questions and ideas for further research.
\end{abstract}




\section{Contents}

1 Introduction $\quad 1$

1.1 Notions and Notations . . . . . . . . . . . . . . . . . . 4

2 The Ultraproduct Construction $\quad 6$

2.1 History . . . . . . . . . . . . . . . . . 6

2.2 Ultrafilters . . . . . . . . . . . . . . . . . . . . . 8

2.3 Ultraproducts . . . . . . . . . . . . . . . . . . . . . . 10

2.4 Ultralimits . . . . . . . . . . . . . . . . . . . . . . . . 12

2.5 The Banach Space Case . . . . . . . . . . . . . . . . . 13

2.6 Cardinality and Isomorphisms . . . . . . . . . . . . . . . 16

2.7 Passing Parentheses . . . . . . . . . . . . . . . . . . . . . . 19

3 Nice Representative Sequences $\quad 23$

3.1 In Banach and Hilbert Spaces . . . . . . . . . . . . . . 23

3.2 In $\mathrm{C}^{*}$-Algebras $\ldots \ldots \ldots \ldots \ldots$ 
$4 \mathcal{K}\left(\mathcal{H}^{\mathcal{U}}\right), \mathcal{K}(\mathcal{H})^{\mathcal{U}}, \mathcal{B}(\mathcal{H})^{\mathcal{U}}$, and $\mathcal{B}\left(\mathcal{H}^{\mathcal{U}}\right)$

5 Spectra in Ultraproducts

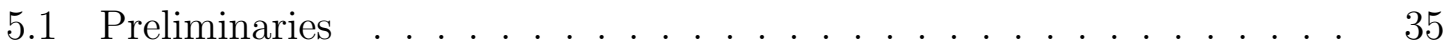

5.2 Pseudospectra . . . . . . . . . . . . . . . . . . . . . 37

5.3 Ultralimits of Sets . . . . . . . . . . . . . . . . . . . . . . 39

5.4 Spectra in Ultraproducts . . . . . . . . . . . . . . . . . 43

6 Lin's Theorem and Ultraproducts $\quad 56$

6.1 Counterexamples ....................... 56

6.2 Lin's Property and Lin's Theorem . . . . . . . . . . . . . . . 58

7 Continuous Logic $\quad 64$

7.1 History . . . . . . . . . . . . . . . . . . . . . 64

7.2 Language for $\mathrm{C}^{*}$-Algebras . . . . . . . . . . . . . . 66

7.3 Theories and Elementary Equivalence . . . . . . . . . . . . . . . . 68

7.4 Continuous Model Theory . . . . . . . . . . . . . . . . 72

7.5 Definability . . . . . . . . . . . . . . . . 74

8 Particular Preserved Properties $\quad 79$

8.1 Axiomatizable and Local Classes _ . . . . . . . . . . . . . . . . . . 79

8.2 Non-Axiomatizable and Non-Local Classes . . . . . . . . . . . . . . . 80

8.3 Unital $\mathrm{C}^{*}$-Algebras Are Axiomatizable . . . . . . . . . . . . . . 82 
8.4 Commutative $\mathrm{C}^{*}$-Algebras Are Axiomatizable . . . . . . . . . . . 84

8.5 Finite $\mathrm{C}^{*}$-Algebras are Axiomatizable . . . . . . . . . . . . 86

8.6 Stable Rank One is Axiomatizable . . . . . . . . . . . . . . . . . . . 89

8.7 Unitary Polar Decompositions . . . . . . . . . . . . . . . . . . . . . 91

8.8 Real Rank Zero is Axiomatizable . . . . . . . . . . . . . . . . . . . . 92

8.9 Tracial C*-Algebras are Axiomatizable . . . . . . . . . . . . . . 95

9 Pseudocompact and Pseudomatrical C*-Algebras 96

9.1 Definition of Pseudocompactness _. . . . . . . . . . . . . . 96

9.2 Stability . . . . . . . . . . . . . . . . . . . . . 98

10 Commutative Pseudocompact $\mathrm{C}^{*}$-Algebras 101

10.1 Commutative $\mathrm{C}^{*}$-Algebras . . . . . . . . . . . . . . 102

10.2 Ultraproducts of Commutative $\mathrm{C}^{*}$-Algebras $\ldots \ldots \ldots$

10.3 Commutative Pseudocompact $\mathrm{C}^{*}$-Algebras . . . . . . . . . . . 105

11 Properties of Pseudocompact C*-Algebras 113

11.1 Unitaries and $K_{1} \ldots \ldots \ldots \ldots \ldots \ldots$

11.2 Minimal Projections . . . . . . . . . . . . . . . . . 115

11.3 Approximate Fractions of Projections . . . . . . . . . . . . . . . . 118

11.4 The Dixmier Property . . . . . . . . . . . . . . . . . . . . . . 121

12 Properties of Pseudomatricial C*-Algebras 127 
12.1 Trivial Centers . . . . . . . . . . . . . . . . . . . . 127

12.2 Highly Irreducible Elements . . . . . . . . . . . . . . . . . . 128

12.3 Commutators . . . . . . . . . . . . . . . . . . . 129

12.4 Hyponormality . . . . . . . . . . . . . . . . . . . 135

12.5 Projections and Traces . . . . . . . . . . . . . . . 136

$12.6 K_{0}$ Groups . . . . . . . . . . . . . . . . . . . . 143

12.7 Other Finiteness Properties _. . . . . . . . . . . . . . . . 146

13 Pseudocompact Embeddable Algebras $\quad 148$

13.1 MF Algebras . . . . . . . . . . . . . . . . . . . . . . 148

13.2 Embeddings . . . . . . . . . . . . . . . . . . . . . 153

14 Summary and Open Questions $\quad 157$ 


\section{Chapter 1}

\section{Introduction}

Finite-dimensional $\mathrm{C}^{*}$-algebras are $*$-isomorphic to finite direct sums of matrix algebras over the complex numbers. Since matrices over the complex numbers are well-understood and tractable, it is not surprising that some of the earliest classes of $\mathrm{C}^{*}$-algebras which were studied and classified were limits of finite-dimensional $\mathrm{C}^{*}$-algebras. First we have the compact operators on a Hilbert space, which are norm limits of finite-rank operators. Next we have the uniformly hyperfinite or UHF algebras studied by Glimm [Gli60]. UHF algebras are inductive limits of matrix algebras with unital maps. UHF algebras are classified by their supernatural number, which records the prime divisors of the dimensions of the matrix algebras used. Finally, we have the approximately finite-dimensional or AF algebras, a generalization of the UHF algebras studied by Bratteli [Bra72]. These are inductive limits of finite-dimensional $\mathrm{C}^{*}$-algebras, equivalently, they are separable and any finite subset 
is "almost contained" in some finite-dimensional $\mathrm{C}^{*}$-subalgebra. The AF algebras can be represented by a Bratteli diagram, and they are classified by their ordered $K_{0}$ group with order unit by a famous result of Elliott [Ell76], which instigated the famous Elliott program.

We are interested in a different type of limit of finite-dimensional $\mathrm{C}^{*}$-algebras, namely the logical limits of finite-dimensional $\mathrm{C}^{*}$-algebras. These are called the pseudocompact $\mathrm{C}^{*}$-algebras. We also study the logical limits of matrix algebras, which we call the pseudomatricial $\mathrm{C}^{*}$-algebras.

In Chapter 1 we self-referentially refer to Chapter 1 . In Chapter 2, we review ultrafilters and the classical and Banach-space ultraproduct constructions. In Chapter 3 we collect some results about when elements in ultraproducts have nice representative sequences. We show that there are nice representative sequences of orthonormal systems in ultraproducts of Hilbert spaces in [3.1.2]. In Chapter 4 we study the relationships between the $\mathrm{C}^{*}$-algebras $\mathcal{K}\left(\mathcal{H}^{\mathcal{U}}\right), \mathcal{K}(\mathcal{H})^{\mathcal{U}}, \mathcal{B}(\mathcal{H})^{\mathcal{U}}$, and $\mathcal{B}\left(\mathcal{H}^{\mathcal{U}}\right)$. We show that these algebras are all distinct when $\mathcal{H}$ is infinite-dimensional. We show that projections in ultraproducts are either have finite rank or uncountable rank in [4.0.5]. In Chapter 5 we study the relationship between the spectrum of an element $\left(x_{i}\right)_{\mathcal{U}}$ in an ultraproduct and the spectra of the $x_{i}$. We introduce a notion of an ultralimit of sets in [5.3.1] and use it to prove some results, most notably [5.4.3] and [5.4.12]. In [5.4.17] we give a nice way to calculate the spectrum of normal elements which have a normal representative sequence. In Chapter 6, we study how Lin's Theo- 
rem is related to finding normal representative sequences for normal elements in an ultraproduct, see [6.2.4]. In Chapter 7, we review continuous logic and the major model-theoretical results we will need. We show in [7.3.8] that all the algebras of compact operators on infinite-dimensional Hilbert spaces are elementarily equivalent. In Chapter 8 we review some known axiomatizable properties, like being unital, tracial, Dedekind finite, real rank zero, and stable rank one. We show in [8.7.3] that having unitary polar decompositions is axiomatizable. In Chapter 9 we give several equivalent definitions of pseudocompact and pseudomatricial $\mathrm{C}^{*}$-algebras. We show in [9.2.1] that corners of pseudocompact $\mathrm{C}^{*}$-algebras are pseudocompact. In Chapter 10 we summarize work of Henson/Moore and Eagle/Vignati classifying the commutative pseudocompact $\mathrm{C}^{*}$-algebras. We give an explicit axiomatization of the commutative pseudocompact $\mathrm{C}^{*}$-algebras in [10.3.7], and we show this class is not closed under subalgebras or quotients in [10.3.8]. In Chapter 11 we discuss some deeper properties of pseudocompact $\mathrm{C}^{*}$-algebras. We show in [11.1.3] that pseudocompact $\mathrm{C}^{*}$-algebras have trivial $K_{1}$ groups. In pseudocompact $\mathrm{C}^{*}$-algebras every non-zero projection dominates a minimal projection, and modulo minimal projections every projection can be split into approximate fractions, see [11.2.3] and [11.3.1]. We show in [11.4.4] that pseudocompact $\mathrm{C}^{*}$-algebras have the Dixmier property. We show in [11.4.7] that the center of a pseudocompact $\mathrm{C}^{*}$-algebra is pseudocompact. We show there are uncountably many *-isomorphism classes of separable pseudomatricial $\mathrm{C}^{*}$-algebras in [11.3.3]. In [11.3.2] we show that UHF algebras and the unitization of the compacts are not 
pseudocompact. In Chapter 12 we specifically study the class of pseudomatricial $\mathrm{C}^{*}$ algebras. We show in [12.1.1] and [12.5.4] that pseudomatricial algebras have trivial centers and a unique tracial state. We show that all projections in a pseudomatricial $\mathrm{C}^{*}$-algebra are comparable [12.5.1], that all notions of equivalence coincide [12.5.8], and pseudomatricial $\mathrm{C}^{*}$-algebras have strict comparison of projections [12.5.7]. We show in $[12.5 .3]$ that infinite-dimensional pseudomatricial $\mathrm{C}^{*}$-algebras are never simple. We give some idea of what the $K_{0}$ groups of pseudomatricial $\mathrm{C}^{*}$-algebras look like, and show in [12.6.3] that separable infinite-dimensional $\mathrm{C}^{*}$-algebras can have many Archimedean classes of projections. We also show in [12.3.6] that in pseudomatricial $\mathrm{C}^{*}$-algebras, an element is a self-commutator if and only if it is self-adjoint and has trace zero, but in [12.4.3] we see that hyponormal elments need not be normal. In Chapter 13 we discuss the subalgebras of pseudocompact $\mathrm{C}^{*}$-algebras. We show in [13.2.1] and [13.2.3] that pseudocompact $\mathrm{C}^{*}$-algebras and the unitization of the compacts can always be unitally embedded into some pseudomatricial $\mathrm{C}^{*}$-algebra. Finally, in Chapter 14 we summarize our results and conclude with questions and ideas for further research.

\subsection{Notions and Notations}

$M_{n}$ denotes the $n \times n$ matrices over the complex numbers. $\mathcal{H}$ is used to denote a Hilbert space, and $\xi$ and $\eta$ denote vectors. $\mathcal{K}(\mathcal{H})$ and $\mathcal{B}(\mathcal{H})$ denote the compact and bounded linear operators on $\mathcal{H}$ respectively. We use $\mathfrak{A}$ and $\mathfrak{B}$ to denote $\mathrm{C}^{*}$-algebras. 
Given a $C^{*}$-algebra $\mathfrak{A}$ we denote the closed unit ball by $\mathfrak{A}_{\leq 1}$, the center by $\mathcal{Z}(\mathfrak{A})$, the self-adjoint elements by $\mathfrak{A}_{s a}$, the unitary elements by $U(\mathfrak{A})$, and the invertible elements by $\operatorname{GL}(\mathfrak{A})$. The spectrum of an operator $x$ is denoted $\sigma(x)$. We have the bad habit of using $i$ both as an index and as the square root of negative one, but the meaning should be clear in context. Other notation will be introduced as needed. 


\section{Chapter 2}

\section{The Ultraproduct Construction}

\subsection{History}

Although ultraproducts and their techniques are usually considered to be modeltheoretical in nature, analytical questions motivated the definitions of ultrafilters and ultraproducts. Ultrafilters on cardinals were probably introduced by Riesz in [Rie08]. Ulam used the idea in [Ula29] to prove the existence of a set-valued function $F$ defined on the power set of some set $S$ which satisfied "subtractivity" but not "countable additivity". Tarski introduced the general ultrafilter construction [Tar30] and used it to construct a finitely-additive non-atomic $\{0,1\}$-valued measure on an arbitrary set, motivated by Hausdorff's measure problem [Hau14] and Banach's modification of it [Ban23]. Ultraproducts were implicitly used by Skolem [Sko34] to study nonstandard models of arithmetic, and Hewitt [Hew48] to study nonstandard analysis. 
Łoś introduced the construction and fundamental theorem of ultraproducts for algebraic systems under the name "champ logique" or logic field [Łoś55]. Chang and Morel introduced a similar construction (considering a direct product modulo the filter of cofinite sets) in [CM58]. Tarski suggested that the more general "reduced direct product" construction of direct products modulo filters might lead to a proof of the completeness theorem in model theory [FMS63b]. Using Tarski's suggestions, Frayne and Morel introduced the modern definition of ultraproduct (under the name "prime (reduced direct) product") and gave a proof of the compactness theorem from model theory in [FMS58], [FS58], [FST58]. The term "ultraproduct" first appears in [FST58] and seems to be due to Tarski. The modern version of Łos' theorem appear in a paper by Frayne, Morel, and Scott [FMS63a]. An ultraproduct construction on finite $\mathrm{II}_{1}$ factors was introduced independently by McDuff [McD70] and Janseen [Jan72]. However the ideas had appeared earlier in [Sak62] and [Wri54]. The Banach space ultraproduct is due to Dacunha-Castelle and Krivine [DCK72]. The nonstandard hull of a Banach space, a similar construction, is due to Luxemburg [Lux69]. For a good survey of results about ultraproducts of Banach spaces, see Stefan Heinrich's paper [Hei80]. Ultraproducts of operator algebras were studied by Li [Li05]. A non-standard hull construction was introduced by Baratella and Ng [BN09], [BN11]. The model theory of operator algebras has been studied recently by Farah, Hart and Sherman [FHS13], [FHS14a], [FHS14b]. 


\section{$2.2 \quad$ Ultrafilters}

Definition 2.2.1. We say $\mathcal{U}$ is an ultrafiter on an infinite index set $I$ if $\mathcal{U} \subseteq \mathcal{P}(I)$ is a collection of subsets of $I$ satisfying

1. $I \in \mathcal{U}, \emptyset \notin \mathcal{U}$.

2. If $A$ and $B \in \mathcal{U}$, then $A \cap B \in \mathcal{U}$.

3. If $A \in \mathcal{U}$ and $A \subseteq B$, then $B \in \mathcal{U}$.

4. For all $A \subseteq I$, either $A \in \mathcal{U}$ or $I \backslash A \in \mathcal{U}$.

If $\mathfrak{F}$ satisfies (1), (2), and (3), it is called a filter. Intuitively filters are a collection of big sets-they strain out the small sets. The assumptions say the whole set $I$ is a big set, the intersection of big sets is a big set, and if you contain a big set you are a big set. Complements of large sets are thought of as small sets. Thus the empty set is a small set, finite unions of small sets are small sets, and if you are contained in a small set then you are a small set. The standard example of a filter on an infinite set is $\mathfrak{F}_{\text {cofinite }}$, the filter of sets with finite complement. The filter $\mathfrak{F}_{\text {cofinite }}$ is called the filter of cofinite sets or the Fréchet filter. Filters are naturally partially ordered by containment.

See, e.g. the articles [Com77] and [Com80] and the book by Comfort and Negrepontis [CN74] for general results about ultrafilters. 
Given an ultrafilter $\mathcal{U}$ we get a finitely-additive probability measure on $I$ by

$$
\mu_{\mathcal{U}}(A)= \begin{cases}1 & \text { if } A \in \mathcal{U} \\ 0 & \text { if } I \backslash A \in \mathcal{U}\end{cases}
$$

Because of this, we sometimes say a property holds $\mathcal{U}$-almost everywhere if it holds for a set in the ultrafilter, or we might say $\mathcal{U}$-many of the components have that property.

The following well-known result allows us to enlarge a non-maximal filter:

Proposition 2.2.2. If $\mathfrak{F}$ is a filter on $I$ and $I_{0} \subseteq I$ is a set with $I_{0} \notin \mathfrak{F}$ and $I \backslash I_{0} \notin \mathfrak{F}$, the collection

$$
\hat{\mathfrak{F}}=\mathfrak{F} \cup\left\{A \cap F \mid A \supseteq I_{0}, F \in \mathfrak{F}\right\}
$$

is a filter on $I$ which dominates $\mathfrak{F}$. In particular an ultrafilter is a maximal filter.

Definition 2.2.3. An ultrafilter $\mathcal{U}$ is called principal, or trivial, if it is determined by a single element $i_{0} \in I$, i.e. $A \in \mathcal{U}$ if and only if $i_{0} \in A$. Such an ultrafilter is denoted $\mathcal{U}_{i_{0}}$. In this case the measure $\mu_{\mathcal{U}_{i}}$ is just a point mass at $i_{0}$. A non-principal ultrafilter $\mathcal{U}$ is called free or non-trivial. This is equivalent to $\bigcap_{A \in \mathcal{U}} A=\emptyset$.

Generally we will only be interested in free ultrafilters.

Definition 2.2.4. An ultrafilter $\mathcal{U}$ is called countably incomplete or countably cofinal if there is a sequence of elements $I_{k}$ of $\mathcal{U}$

$$
I_{1} \supseteq I_{2} \supseteq I_{3} \supseteq \ldots \quad \text { and } \quad \bigcap_{k=1}^{\infty} I_{k}=\emptyset \text {. }
$$


A non-countably complete ultrafilter is called $\aleph_{1}$-complete.

Every free ultrafilter on the set of natural numbers is countably incomplete. Countably incomplete ultrafilters are convenient since they allow the use of standard $1 / n$ approximation arguments. The following result is well-known:

Theorem 2.2.5. Assuming the Axiom of Choice, every filter is dominated by an ultrafilter. In particular, if we start with the filter of cofinite sets we acquire a free ultrafilter.

An alternative way to view ultrafilters is to give $I$ the discrete topology, then ultrafilters correspond to elements of the Stone-Čech compactification $\beta I$. The corresponding topology on ultrafilters is generated by the basis of sets of the form

$$
U_{X}=\{\mathcal{U} \text { an ultrafilter on } I \mid X \in \mathcal{U}\}
$$

The embedding of $I$ into $\beta I$ sends $i_{0} \in I$ to the principal ultrafilter $\mathcal{U}_{i_{0}}$ generated by $i_{0}$, so free ultrafilters can be thought of as elements of $\beta I \backslash I$.

\section{$2.3 \quad$ Ultraproducts}

Definition 2.3.1. Let $A_{i}$ be an arbitrary collection of (non-empty) sets indexed by $I$, and let $\mathcal{U}$ be an ultrafilter on $I$. Consider the Cartesian product $\prod_{i \in I} A_{i}$. Define the equivalence relation $\equiv_{\mathcal{U}}$ on $\prod_{i \in I} A_{i}$ by $\left(a_{i}\right) \equiv_{\mathcal{U}}\left(b_{i}\right)$ if and only if $\left\{i \in I \mid a_{i}=b_{i}\right\} \in \mathcal{U}$.

We define the classical or set-theoretic ultraproduct of the family $\left(A_{i}\right)_{i \in I}$ with respect to the ultrafilter $\mathcal{U}$ to be $\left(\prod_{i \in I} A_{i}\right) / \equiv_{\mathcal{U}}$, which is sometimes denoted $\prod_{i \in I} A_{i} / \mathcal{U}$ 
or $\prod_{\mathcal{U}} A_{i}$. We denote the equivalence class of a sequence $\left(a_{i}\right)$ in the ultraproduct as $\left(a_{i}\right)_{\mathcal{U}}$, and we call $\left(a_{i}\right)$ a representative sequence for that element. We do abusively use the term sequence even when the index set is not countable or given an order. When it is necessary to be pedantic about the index in play, we will write $\prod_{i \rightarrow \mathcal{U}} \mathfrak{A}_{i}$ and elements as $\left(a_{i}\right)_{i \rightarrow \mathcal{U}}$. If all the $\mathfrak{A}_{i}$ are the same, we call this the set-theoretic ultrapower and denote it by $\mathfrak{A}^{\mathcal{U}}$.

A (set-theoretic) ultraproduct of Banach spaces is a vector under component-wise addition and scalar multiplication (it is straight-forward to show these operations are well-defined, i.e. independent of the choice of representative sequences), but it is typically not a Banach space or even a metric space. If the Banach spaces have multiplication, the ultraproduct of the Banach spaces has multiplication by $\left(a_{i}\right)_{\mathcal{U}} \cdot\left(b_{i}\right)_{\mathcal{U}}=\left(a_{i} \cdot b_{i}\right)_{\mathcal{U}}$. Again it is straightforward to check that this is well-defined.

Example 2.3.2. Let $\mathcal{U}$ be a free ultrafilter on $\mathbb{N}$. Then the classical ultrapower $\mathbb{R}^{\mathcal{U}}$ is a model of the hyperreals. It is a field which contains $\mathbb{R}$ as equivalence classes of constant sequences. We define the order by $\left(a_{n}\right)_{\mathcal{U}}<\left(b_{n}\right)_{\mathcal{U}}$ if and only if $\left\{n \in \mathbb{N} \mid a_{n}<\right.$ $\left.b_{n}\right\} \in \mathcal{U}$. The hyperreals contain infinitesimal and infinite elements, like $\left(\frac{1}{n}\right) \mathcal{U}$ and $(n)_{\mathcal{U}}$

Instead of pursuing non-standard analysis, we want to modify the ultraproduct construction to get a Banach space. 


\subsection{Ultralimits}

Definition 2.4.1. Given an ultrafilter $\mathcal{U}$ on $I$, we can define the ultralimit with respect to $\mathcal{U}$ of a sequence $\left(x_{i}\right)_{i \in I}$ in a topological space $X$ by $\lim _{\mathcal{U}} x_{n}=x$ if and only if for all open sets $U$ containing $x,\left\{i \in I \mid x_{i} \in U\right\} \in \mathcal{U}$. If it is necessary to be pedantic about the indices involved we will write $\lim _{i \rightarrow \mathcal{U}} x_{i}$.

Note that if $\mathcal{U}$ is a free ultrafilter on $\mathbb{N}$, then the ultralimit of a sequence of complex numbers extends our the standard definition of a limit:

$$
\begin{aligned}
& \lim _{n \rightarrow \infty} x_{n}=x \quad \text { if and only if } \quad \forall \varepsilon>0 \quad \exists N \in \mathbb{N} \quad n \geq N \Rightarrow\left|x-x_{n}\right|<\varepsilon \\
& \text { if and only if } \quad \forall \varepsilon>0 \quad\left\{n:\left|x-x_{n}\right|<\varepsilon\right\} \in \mathfrak{F}_{\text {cofinite }} .
\end{aligned}
$$

In particular, if $\lim _{n \rightarrow \infty} x_{n}=x$, then $\lim _{\mathcal{U}} x_{n}=x$ as well.

The following result is well-known:

Proposition 2.4.2 (e.g. 1.5 in [Hei80]). Let $K$ be a compact Hausdorff space. Then for each sequence $\left(x_{i}\right)_{i \in I}$ in $\prod_{i \in I} K$ the $\operatorname{limit}_{\lim } x_{i}=x$ exists in $K$ and it is unique.

We know that every convergent sequence of complex numbers is bounded. The previous result shows conversely that every bounded sequence of complex numbers has an ultralimit. The following result catalogues the well-known properties of ultralimits:

Proposition 2.4.3 (Properties of Ultralimits). Suppose $\left(a_{i}\right)_{i \in I}$ and $\left(b_{i}\right)_{i \in I}$ are sequences in $\ell_{\infty}(I, \mathbb{C})$, so that $\left\|\left(a_{i}\right)\right\|_{\infty}=\sup _{i \in I}\left|a_{i}\right|<\infty,\left\|\left(b_{i}\right)\right\|_{\infty}=\sup _{i \in I}\left|b_{i}\right|<\infty$. Then we have the following: 
1. $\lim _{\mathcal{U}} a_{i}+\lim _{\mathcal{U}} b_{i}=\lim _{\mathcal{U}}\left(a_{i}+b_{i}\right)$.

2. If $\lim _{\mathcal{U}} a_{i}=0$, then $\lim _{\mathcal{U}}\left(a_{i} \cdot b_{i}\right)=0$.

3. $\left(\lim _{\mathcal{U}} a_{i}\right)\left(\lim _{\mathcal{U}} b_{i}\right)=\lim _{\mathcal{U}}\left(a_{i} \cdot b_{i}\right)$.

4. If $a_{i} \geq 0$ for all $i \in I$, then $\lim _{\mathcal{U}} a_{i} \geq 0$.

5. If $f: \mathbb{C} \rightarrow \mathbb{C}$ is a continuous function then $\lim _{\mathcal{U}} f\left(a_{i}\right)=f\left(\lim _{\mathcal{U}} a_{i}\right)$.

Notice that ultralimits are not shift invariant. For example, if our ultrafilter contains the odd numbers, $\lim _{\mathcal{U}}(-1)^{n}=-1$ but $\lim _{\mathcal{U}}(-1)^{n+1}=1$. Compare this to Cesàro and Banach limits, which are shift-invariant but not multiplicative.

\subsection{The Banach Space Case}

Definition 2.5.1. Let $\left(E_{i}\right)_{i \in I}$ be a family of Banach spaces. Consider the set

$$
\ell_{\infty}\left(I, E_{i}\right)=\left\{\left(x_{i}\right)_{i \in I} \in \prod_{i \in I} E_{i}:\left\|\left(x_{i}\right)_{i \in I}\right\|_{\infty}:=\sup _{i \in I}\left\|x_{i}\right\|_{E_{i}}<\infty\right\} .
$$

Then $\ell_{\infty}\left(I, E_{i}\right)$ is a Banach space under component-wise addition and scalar multiplication:

$$
\left(x_{i}\right)_{i \in I}+\left(y_{i}\right)_{i \in I}:=\left(x_{i}+y_{i}\right)_{i \in I} \quad \text { and } \quad \lambda\left(x_{i}\right)_{i \in I}:=\left(\lambda x_{i}\right)_{i \in I} .
$$

Definition 2.5.2. For $\mathcal{U}$ an ultrafilter on $I$, let

$$
N_{\mathcal{U}}=\left\{\left(x_{i}\right) \in \ell_{\infty}\left(I, E_{i}\right): \lim _{\mathcal{U}}\left\|x_{i}\right\|_{E_{i}}=0\right\}
$$


Then $N_{\mathcal{U}}$ is a closed subspace in $\ell_{\infty}\left(I, E_{i}\right)$. If the $E_{i}$ are Banach algebras, then $N_{\mathcal{U}}$ is a closed, two-sided ideal.

Definition 2.5.3. Let $\left(E_{i}\right)_{i \in I}$ be a family of Banach spaces. We define the Banach space ultraproduct of the $\left(E_{i}\right)_{i \in I}$ with respect to an ultrafilter $\mathcal{U}$ on $I$ by

$$
\prod_{\mathcal{U}} E_{i}=\ell_{\infty}\left(I, E_{i}\right) / N_{\mathcal{U}}
$$

We will denote the equivalence class of an element $\left(x_{i}\right)_{i \in I} \in \ell_{\infty}\left(I, E_{i}\right)$ in the ultraproduct as $\left(x_{i}\right)_{\mathcal{U}}$. We equip the ultraproduct $\prod_{\mathcal{U}} E_{i}$ with the canonical quotient norm:

$$
\left\|\left(x_{i}\right)_{\mathcal{U}}\right\|=\inf _{\left(a_{i}\right) \in N_{\mathcal{U}}}\left\|\left(x_{i}-a_{i}\right)_{i \in I}\right\|_{\infty}=\inf _{\left(a_{i}\right) \in N_{\mathcal{U}}} \sup _{i \in I}\left\|x_{i}-a_{i}\right\|_{E_{i}}
$$

In other words, ultraproducts are bounded sequences modulo null sequences, but with respect to an ultralimit rather than our standard definition of limit. This is similar to considering $\ell_{\infty} / c_{0}$ or the direct product modulo the direct sum.

From now on ultraproducts of Banach spaces will be assumed to be the Banach space ultraproduct unless otherwise noted. The following well-known result allows us to compute the norms of elements in ultraproducts easily:

Proposition 2.5.4. The norm of $\left(x_{i}\right)_{\mathcal{U}}$ in $\prod_{\mathcal{U}} E_{i}$ can be computed as

$$
\left\|\left(x_{i}\right)_{\mathcal{U}}\right\|=\inf _{\left(a_{i}\right) \in N_{\mathcal{U}}} \sup _{i \in I}\left\|x_{i}-a_{i}\right\|_{E_{i}}=\lim _{\mathcal{U}}\left\|x_{i}\right\|
$$

Note that if $\mathcal{U}_{i_{0}}$ is the trivial or principal ultrafilter generated by $i_{0} \in I$ then $\prod_{\mathcal{U}_{i_{0}}} E_{i} \cong E_{i_{0}}$. There is always canonical isometric embedding $J$ of $E$ into its ultrapower $E^{\mathcal{U}}$ which is defined by $J(x)=\left(x_{i}\right)_{\mathcal{U}}$ where $x_{i}=x$ for all $i \in I$. 
Now that we have introduced the ultraproduct of Banach spaces, we can define the ultraproduct of operators:

Proposition 2.5.5 (e.g. 2.2 in [Hei80]). Let $\left(E_{i}\right)_{i \in I}$ and $\left(F_{i}\right)_{i \in I}$ be families of Banach spaces indexed by the same set $I$, and for each $i \in I, T_{i} \in \mathcal{B}\left(E_{i}, F_{i}\right)$ is a bounded linear map from $E_{i}$ to $F_{i}$, such that $\sup _{i \in I}\left\|T_{i}\right\|<\infty$. The ultraproduct of the family of operators $\left(T_{i}\right)_{i \in I}$ with respect to the ultrafilter $\mathcal{U}$ on $I$ is $\left(T_{i}\right)_{\mathcal{U}}: \prod_{\mathcal{U}} E_{i} \rightarrow \prod_{\mathcal{U}} F_{i}$ defined by

$$
\left(x_{i}\right) \mapsto\left(T_{i} x_{i}\right)_{\mathcal{U}}
$$

This map is well-defined, and $\left\|\left(T_{i}\right)_{\mathcal{U}}\right\|=\lim _{\mathcal{U}}\left\|T_{i}\right\|$.

Proposition 2.5.6 (3.1 in [Hei80]).

1. Banach algebras are stable under ultraproducts (with component-wise multiplication).

2. $C^{*}$-algebras are stable under ultraproducts (with component-wise adjoints).

Comment: this construction can be modified for tracial von Neumann algebras by using the 2-norm $\|x\|_{2}:=\sqrt{\tau\left(x^{*} x\right)}$. One similarly starts with sequences uniformly bounded in the operator norm, defines $\tau_{\mathcal{U}}\left(\left(x_{i}\right)_{i \in I}\right):=\lim _{\mathcal{U}} \tau\left(x_{i}\right)$, and quotients by the ideal of sequences which vanish under the 2-norm. See [FHS14b].

The following (in the real case) is observed in [BT79] and in general in [GH01]:

Proposition 2.5.7. If for all $i \in I, \mathcal{H}_{i}$ is a Hilbert space with inner product $\langle\cdot \mid \cdot\rangle_{i}$, then $\prod_{\mathcal{U}} \mathcal{H}_{i}$ is a Hilbert space with inner product $\left\langle\left(\xi_{i}\right)_{\mathcal{U}} \mid\left(\eta_{i}\right)_{\mathcal{U}}\right\rangle_{\mathcal{U}}:=\lim _{\mathcal{U}}\left\langle\xi_{i} \mid \eta_{i}\right\rangle_{i}$ 


\subsection{Cardinality and Isomorphisms}

The following is a well-known fact:

Proposition 2.6.1 (e.g. 3.1 in [BT79]). If $\mathfrak{A}$ is a finite-dimensional $\mathrm{C}^{*}$-algebra, then $\mathfrak{A}$ is $*$-isomorphic to any of its ultrapowers $\mathfrak{A}^{\mathcal{U}}$.

Proof. Let $\left(x_{n}\right)_{\mathcal{U}} \in \mathfrak{A}^{\mathcal{U}}$, then there is an $M$ such that $\left\|x_{n}\right\| \leq M$. Since $\mathfrak{A}$ is finitedimensional, the ball of radius $M$ centered at zero is compact in $\mathfrak{A}$, so $\lim _{\mathcal{U}} x_{n}=x$ exists in $\mathfrak{A}$. Since $\lim _{\mathcal{U}}\left\|x_{n}-x\right\|=0$, the constant sequence $(x)_{\mathcal{U}}$ is equal to $\left(x_{n}\right)_{\mathcal{U}}$. In particular the embedding $J: \mathfrak{A} \rightarrow \mathfrak{A}^{\mathcal{U}}$ sending $x \mapsto(x)_{\mathcal{U}}$ is a $*$-isomorphism.

This is not surprising: ultraproducts usually follow a finite-or-highly-infinite dichotomy. There is a classical result that if $\mathcal{U}$ is a free ultrafilter on the natural numbers, and for all $n \in \mathbb{N}, A_{n}$ is a subset of the natural numbers, then the settheoretical ultraproduct $\prod_{\mathcal{U}} A_{n}$ is either finite or uncountable. This result seems to have first appeared as Theorem 1.28 and 1.29 in [FMS63a]. The analogous property for Banach spaces says that the ultraproduct of Banach spaces (with respect to a free ultrafilter on the natural numbers) is either finite-dimensional or non-separable:

Theorem 2.6.2 (5.2 in [FHS13], 3.1 in [BT79], and 5.2 in [GH01]). Let $\mathcal{U}$ be a free ultrafilter on the natural numbers. Suppose $\mathfrak{A}_{n}$ are $\mathrm{C}^{*}$-algebras. Then $\prod_{\mathcal{U}} \mathfrak{A}_{n}$ is separable if and only if there is a finite $d$ such that $\left\{n \mid \mathfrak{A}_{n}\right.$ is $d$-dimensional $\} \in \mathcal{U}$, in which case $\prod_{\mathcal{U}} \mathfrak{A}_{n}$ is $d$-dimensional.

However, strange things can happen for $\aleph_{1}$-complete ultrafilters: 
Proposition 2.6.3 (6.3 in [GH01]). The following are equivalent:

1. There exists a measurable cardinal.

2. There is a free, $\aleph_{1}$-complete ultrafilter $\mathcal{U}$ and a separable infinite-dimensional $\mathrm{C}^{*}$-algebra $\mathfrak{A}$ so $\mathfrak{A}^{\mathcal{U}}$ is separable.

The following result allows us to treat an iterated ultrapower as an ultrapower of the base space. This result is not surprising since ultraproducts are a sort of limit and limit points of limit points are limit points.

Proposition 2.6.4 ( $\S 13$ in $[\operatorname{Sim} 82])$. If $\mathcal{U}$ is an ultrafilter on $I$ and $\mathcal{V}$ is an ultrafilter on $J$, then

$$
\prod_{j \rightarrow \mathcal{V}}\left(\prod_{i \rightarrow \mathcal{U}} \mathfrak{A}_{i, j}\right) \cong \prod_{(i, j) \rightarrow \mathcal{U} \times \mathcal{V}} \mathfrak{A}_{i, j}
$$

where $\mathcal{U} \times \mathcal{V}$ is the ultrafilter on $I \times J$ defined by

$$
S \in \mathcal{U} \times \mathcal{V} \quad \Longleftrightarrow \quad S \subseteq I \times J \text { and }\{j \in J \mid\{i \in I \mid(i, j) \in S\} \in \mathcal{U}\} \in \mathcal{V}
$$

In fact, if either of $\mathcal{U}$ or $\mathcal{V}$ is non-trivial, then so is $\mathcal{U} \times \mathcal{V}$. If either of $\mathcal{U}$ or $\mathcal{V}$ is countably incomplete, then so is $\mathcal{U} \times \mathcal{V}$.

Corollary 2.6.5. For any $C^{*}$-algebra $\mathfrak{A}$ and ultrafilters $\mathcal{U}$ and $\mathcal{V},\left(\mathfrak{A}^{\mathcal{U}}\right)^{\mathcal{V}} \cong \mathfrak{A}^{\mathcal{U} \times \mathcal{V}}$.

However, note that the ultrafilters $\mathcal{U} \times \mathcal{V}$ and $\mathcal{V} \times \mathcal{U}$ are different in general.

Question 2.6.6. Is it the case that $\left(\mathfrak{A}^{\mathcal{U}}\right)^{\mathcal{V}} \cong \mathfrak{A}^{(\mathcal{U} \times \mathcal{V})} \cong \mathfrak{A}^{(\mathcal{V} \times \mathcal{U})} \cong\left(\mathfrak{A}^{\mathcal{V}}\right)^{\mathcal{U}}$ ? 
Only the middle isomorphism is in question. In other words, do ultrapowers commute? The corresponding question for ultraproducts is false: let

$$
\mathfrak{A}_{i, j}= \begin{cases}\mathbb{C}, & \text { if } i \text { is odd } \\ 0 & \text { if } i \text { is even }\end{cases}
$$

Then let $\mathcal{U}$ and $\mathcal{V}$ be ultrafilters so $\mathcal{U}$ contains the set of even numbers and $\mathcal{V}$ contains the set of odd numbers. Then

$$
\prod_{i \rightarrow \mathcal{U}} \prod_{j \rightarrow \mathcal{V}} \mathfrak{A}_{i, j} \cong \mathbb{C} \quad \text { but } \quad \prod_{j \rightarrow \mathcal{V}} \prod_{i \rightarrow \mathcal{U}} \mathfrak{A}_{i, j} \cong\{0\}
$$

There are some cases where it is known that different ultrafilters yield isomorphic ultrapowers:

Definition 2.6.7. The relative commutant of a $\mathrm{C}^{*}$-algebra $\mathfrak{A}$ in an ultrapower $\mathfrak{A}^{\mathcal{U}}$ is

$$
\mathfrak{A}^{\prime} \cap \mathfrak{A}^{\mathcal{U}}=\left\{\left(b_{i}\right)_{\mathcal{U}}: \forall a \in \mathfrak{A} \quad\left(a b_{i}\right)_{\mathcal{U}}=\left(b_{i} a\right)_{\mathcal{U}}\right\}
$$

Theorem (5.1 in [FHS13], 3.4 in [GH01]). Let $\mathfrak{A}$ be an infinite-dimensional, separable, unital $\mathrm{C}^{*}$-algebra. Then the following are equivalent:

1. For all nonprincipal ultrafilters $\mathcal{U}$ and $\mathcal{V}$ on $\mathbb{N}$, the ultrapowers $\mathfrak{A}^{\mathcal{U}}$ and $\mathfrak{A}^{\mathcal{V}}$ are isomorphic.

2. For all nonprincipal ultrafilters $\mathcal{U}$ and $\mathcal{V}$ on $\mathbb{N}$, the relative commutants of $\mathfrak{A}$ in $\mathfrak{A}^{\mathcal{U}}$ and $\mathfrak{A}^{\mathcal{V}}$ are isomorphic.

3. For all nonprincipal ultrafilters $\mathcal{U}$ and $\mathcal{V}$ on $\mathbb{N}$, the ultrapowers of the unitary groups $U(\mathfrak{A})^{\mathcal{U}}$ and $U(\mathfrak{A})^{\mathcal{V}}$ are isomorphic. 
4. For all nonprincipal ultrafilters $\mathcal{U}$ and $\mathcal{V}$ on $\mathbb{N}$, the relative commutants of $U(\mathfrak{A})$ in the ultrapowers $U(\mathfrak{A})^{\mathcal{U}}$ and $U(\mathfrak{A})^{\mathcal{V}}$ are isomorphic.

5. The Continuum Hypothesis holds.

If $\mathfrak{A}$ is separable but not unital then (1), (2), and (5) are equivalent. The equivalence of (1) and (2) can fail if $\mathfrak{A}$ is nonseparable.

In fact, it was shown that

Theorem 2.6.8 (8.2 in [FS10]). If the Continuum Hypothesis fails, and $\mathfrak{A}$ is a separable infinite-dimensional $\mathrm{C}^{*}$-algebra, then there are $2^{\mathfrak{c}}$ non-isomorphic ultrapowers of $\mathfrak{A}$ associated to ultrafilters on $\mathbb{N}$, where $\mathfrak{c}$ is the cardinality of the real numbers.

\subsection{Passing Parentheses}

The next well-known result shows that ultraproducts and finite direct sums commute:

Proposition 2.7.1. Let $\mathcal{U}$ be an ultrafilter on $I$ and for all $i \in I$ let $\mathfrak{A}_{i}$ and $\mathfrak{B}_{i}$ be $\mathrm{C}^{*}$-algebras. Then $\left(\prod_{\mathcal{U}} \mathfrak{A}_{i}\right) \oplus\left(\prod_{\mathcal{U}} \mathfrak{B}_{i}\right) \cong \prod_{\mathcal{U}} \mathfrak{A}_{i} \oplus \mathfrak{B}_{i}$. In particular, for any $\mathrm{C}^{*}$-algebras $\mathfrak{A}$ and $\mathfrak{B}$, we have that $(\mathfrak{A} \oplus \mathfrak{B})^{\mathcal{U}} \cong \mathfrak{A}^{\mathcal{U}} \oplus \mathfrak{B}^{\mathcal{U}}$.

Proof. Consider the map from $\left(\prod_{\mathcal{U}} \mathfrak{A}_{i}\right) \oplus\left(\prod_{\mathcal{U}} \mathfrak{B}_{i}\right) \rightarrow \prod_{\mathcal{U}}\left(\mathfrak{A}_{i} \oplus \mathfrak{B}_{i}\right)$ sending

$$
\left(\left(a_{i}\right)_{\mathcal{U}},\left(b_{i}\right)_{\mathcal{U}}\right) \mapsto\left(a_{i}, b_{i}\right)_{\mathcal{U}}
$$

This is well-defined and injective since

$$
\left\|\left(a_{i}, b_{i}\right)_{\mathcal{U}}\right\|=\lim _{\mathcal{U}}\left\|\left(a_{i}, b_{i}\right)\right\|=\lim _{\mathcal{U}} \max \left(\left\|a_{i}\right\|,\left\|b_{i}\right\|\right)=\max \left(\lim _{\mathcal{U}}\left\|a_{i}\right\|, \lim _{\mathcal{U}}\left\|b_{i}\right\|\right)
$$


It is clearly surjective. For linearity we calculate

$$
\begin{aligned}
\left(\left(a_{i}^{(1)}\right) \mathcal{U},\left(b_{i}^{(1)}\right) \mathcal{U}\right)+\lambda\left(\left(a_{i}^{(2)}\right)_{\mathcal{U}},\left(b_{i}^{(2)}\right) \mathcal{U}\right) & =\left(\left(a_{i}^{(1)}+\lambda a_{i}^{(2)}\right)_{\mathcal{U}},\left(b_{i}^{(1)}+\lambda b_{i}^{(2)}\right)_{\mathcal{U}}\right) \\
& \mapsto\left(a_{i}^{(1)}+\lambda a_{i}^{(2)}, b_{i}^{(1)}+\lambda b_{i}^{(2)}\right)_{\mathcal{U}} \\
& =\left(a_{i}^{(1)}, b_{i}^{(1)}\right)_{\mathcal{U}}+\lambda\left(a_{i}^{(2)}, b_{i}^{(2)}\right)_{\mathcal{U}} .
\end{aligned}
$$

For multiplicative, we calculate

$$
\begin{aligned}
\left(\left(a_{i}^{(1)}\right)_{\mathcal{U}},\left(b_{i}^{(1)}\right)_{\mathcal{U}}\right) \cdot\left(\left(a_{i}^{(2)}\right)_{\mathcal{U}},\left(b_{i}^{(2)}\right)_{\mathcal{U}}\right) & =\left(\left(a_{i}^{(1)} a_{i}^{(2)}\right)_{\mathcal{U}},\left(b_{i}^{(1)} b_{i}^{(2)}\right)_{\mathcal{U}}\right) \\
& \mapsto\left(a_{i}^{(1)} a_{i}^{(2)}, b_{i}^{(1)} b_{i}^{(2)}\right)_{\mathcal{U}}=\left(a_{i}^{(1)}, b_{i}^{(1)}\right)_{\mathcal{U}} \cdot\left(a_{i}^{(2)}, b_{i}^{(2)}\right)_{\mathcal{U}} .
\end{aligned}
$$

To check $*$-preserving, we calculate

$$
\left(\left(a_{i}\right)_{\mathcal{U}},\left(b_{i}\right)_{\mathcal{U}}\right)^{*}=\left(\left(a_{i}^{*}\right)_{\mathcal{U}},\left(b_{i}^{*}\right)_{\mathcal{U}}\right) \mapsto\left(a_{i}^{*}, b_{i}^{*}\right)_{\mathcal{U}}=\left(a_{i}, b_{i}\right)_{\mathcal{U}}^{*}
$$

Similarly, there is a well-known result that tensoring by a matrix algebra commutes with ultraproducts:

Proposition 2.7.2. Let $\mathcal{U}$ be an ultrafilter on $I$, and for all $i \in I$ let $\mathfrak{A}_{i}$ be a $\mathrm{C}^{*}$ algebra. Then for all natural numbers $n$,

$$
M_{n}\left(\prod_{\mathcal{U}} \mathfrak{A}_{i}\right) \cong M_{n} \otimes \prod_{\mathcal{U}} \mathfrak{A}_{i} \cong \prod_{\mathcal{U}} M_{n} \otimes \mathfrak{A}_{i} \cong \prod_{\mathcal{U}} M_{n}\left(\mathfrak{A}_{i}\right) .
$$

In particular, $\left(M_{n}(\mathfrak{A})\right)^{\mathcal{U}} \cong M_{n}\left(\mathfrak{A}^{\mathcal{U}}\right)$.

Proof. Consider the map from $M_{n}\left(\prod_{\mathcal{U}} \mathfrak{A}_{i}\right)$ to $\prod_{\mathcal{U}} M_{n}\left(A_{i}\right)$ sending

$$
\left[\left(a_{i}^{(j, k)}\right)_{\mathcal{U}}\right]_{1 \leq j, k \leq n} \mapsto\left(\left[a_{i}^{(j, k)}\right]_{1 \leq j, k \leq n}\right)_{\mathcal{U}}
$$


By a standard argument, for all $i \in I$,

$$
\max _{1 \leq j, k \leq n}\left\|a_{i}^{(j, k)}\right\| \leq\left\|\left[a_{i}^{(j, k)}\right]_{j, k}\right\| \leq n^{2} \max \left\|a_{i}^{(j, k)}\right\|
$$

Thus

$$
\begin{aligned}
\max _{1 \leq j, k \leq n} \lim _{\mathcal{U}}\left\|a_{i}^{(j, k)}\right\| & =\lim _{\mathcal{U}} \max _{1 \leq j, k \leq n}\left\|a_{i}^{(j, k)}\right\| \leq\left\|\left(\left[a_{i}^{(j, k)}\right]_{j, k}\right)_{\mathcal{U}}\right\| \\
& =\lim _{\mathcal{U}}\left\|\left[a_{i}^{(j, k)}\right]_{j, k}\right\| \leq n^{2} \max \left\|a_{i}^{(j, k)}\right\| .
\end{aligned}
$$

Which is sufficient for well-definedness and injectivity. This map is clearly surjective.

For linearity we calculate

$$
\begin{aligned}
{\left[\left(a_{i}^{(j, k)}\right)_{\mathcal{U}}\right]_{j, k}+\lambda\left[\left(b_{n}^{(j, k)}\right)_{\mathcal{U}}\right]_{j, k} } & =\left[\left(a_{i}^{(j, k)}+\lambda b_{i}^{(j, k)}\right)_{\mathcal{U}}\right] \\
& \mapsto\left(\left[a_{i}^{(j, k)}+\lambda b_{i}^{(j, k)}\right]\right)_{\mathcal{U}} \\
& =\left(\left[a_{i}^{(j, k)}\right]\right)_{\mathcal{U}}+\lambda\left(\left[b_{i}^{(j, k)}\right]\right)_{\mathcal{U}} .
\end{aligned}
$$

To check the map is multiplicative, we calculate

$$
\begin{aligned}
{\left[\left(a_{i}^{(j, k)}\right)_{\mathcal{U}}\right]_{j, k} \cdot\left[\left(b_{i}^{(j, k)}\right)_{\mathcal{U}}\right]_{j, k} } & =\left[\left(\sum_{1 \leq \ell \leq n} a_{i}^{(j, \ell)} b_{i}^{(\ell, k)}\right)_{\mathcal{U}}\right]_{j, k} \mapsto\left(\left[\sum_{1 \leq \ell \leq n} a_{i}^{(j, \ell)} b_{i}^{(\ell, k)}\right]_{j, k}\right)_{\mathcal{U}} \\
& =\left(\left[a_{i}^{(j, k)}\right]\right)_{\mathcal{U}} \cdot\left(\left[b_{i}^{(j, k)}\right]_{j, k}\right)_{\mathcal{U}} .
\end{aligned}
$$

For $*$-preserving, we calculate

$$
\left[\left(a_{i}^{(j, k)}\right)_{\mathcal{U}}\right]_{j, k}^{*}=\left[\left(a_{i}^{(k, j)^{*}}\right)_{\mathcal{U}}\right]_{j, k} \mapsto\left(\left[a_{i}^{(k, j)^{*}}\right]_{j, k}\right)_{\mathcal{U}}=\left(\left[a_{i}^{(j, k)}\right]_{j, k}\right)_{\mathcal{U}}^{*}
$$

The operation of tensoring by an infinite-dimensional $\mathrm{C}^{*}$-algebra cannot commute with ultrapowers, see [Gha15]. 
Amenability of ultrapowers has been studied in [Daw09] and [Daw10]. Ultraproducts are important in two major open questions in operator algebras: Connes' embedding problem, which asks if every type $\mathrm{II}_{1}$ factor with separable predual is embeddable into an ultraproduct of the hyperfinite type $\mathrm{II}_{1}$ factor $R$, and Kirchberg's embedding problem, which asks if every separable $\mathrm{C}^{*}$-algebra can be embedded into an ultrapower of the Cuntz algebra $\mathcal{O}_{2}$. See for instance [Oza13] and [GS15b]. 


\section{Chapter 3}

\section{Nice Representative Sequences}

Suppose we know an element $\left(x_{i}\right)_{\mathcal{U}}$ in an ultraproduct has some property $P$. It is natural to ask if we can find a representative sequence $\left(y_{i}\right)$ with $\left(y_{i}\right)_{\mathcal{U}}=\left(x_{i}\right)_{\mathcal{U}}$ so that all of the $y_{i}$ have the property $P$, or $\mathcal{U}$-many of the $y_{i}$ have the property $P$. We will see that these questions can be surprisingly subtle and are closely related to the notions of definability and weakly-stable relations.

\subsection{In Banach and Hilbert Spaces}

The following is a well-known but useful observation:

Proposition 3.1.1. Let $\mathcal{U}$ be an ultrafilter on $I$, and for all $i \in I$ let $E_{i}$ be a nontrivial Banach space. Then for any $\left(x_{i}\right)_{\mathcal{U}} \in \prod_{\mathcal{U}} \mathfrak{A}_{i}$ there is a representative sequence $\left(y_{i}\right)_{\mathcal{U}}=\left(x_{i}\right)_{\mathcal{U}}$ so that $\left\|y_{i}\right\|=\left\|\left(x_{i}\right)_{\mathcal{U}}\right\|$ for all $i$. 
Proof. Suppose $\left(x_{i}\right)_{\mathcal{U}} \in \prod_{\mathcal{U}} E_{i}$ with $\left\|\left(x_{i}\right)_{\mathcal{U}}\right\|=\lambda$. If $\lambda=0$ then $\left(x_{i}\right)_{\mathcal{U}}=(0)_{\mathcal{U}}$. Otherwise let $S=\left\{i:\left\|x_{i}\right\|>\lambda / 2\right\} \in \mathcal{U}$. We let $y_{i}=\lambda x_{i} /\left\|x_{i}\right\|$ for $n \in S$, and $y_{i} \in \mathfrak{A}_{i}$ with $\left\|y_{i}\right\|=\lambda$ for $i \notin S$. Since

$$
\lim _{\mathcal{U}}\left\|y_{i}-x_{i}\right\|=\lim _{\mathcal{U}}\left\|\frac{\lambda}{\left\|x_{i}\right\|} x_{i}-x_{i}|| \leq \lim _{\mathcal{U}}\left|\frac{\lambda}{\left\|x_{i}\right\|}-1\right|\right\| x_{i}\left\|=\lim _{\mathcal{U}}\left|\lambda-\left\|x_{i}\right\|\right|=0,\right.
$$

we have $\left(y_{i}\right)_{\mathcal{U}}=\left(x_{i}\right)_{\mathcal{U}}$ and $\left\|y_{i}\right\|=\lambda$ for all $i \in I$ as claimed. In general there may be a null set for which $\mathfrak{A}_{i}$ are the zero Banach space, but these are safely ignored.

The next result allows us to find nice representative sequences of vectors in ultraproducts of Hilbert spaces.

Proposition 3.1.2. Let $\mathcal{U}$ be an ultrafilter on $I$, and for all $i \in I$ let $\mathcal{H}_{i}$ be a (non-trivial) Hilbert space. Then we have the following:

1. If $\left(\xi_{i}\right)_{\mathcal{U}}$, and $\left(\eta_{i}\right)_{\mathcal{U}}$ are orthogonal in $\prod_{\mathcal{U}} \mathcal{H}_{i}$, then we can find $\left(\xi_{i}^{\prime}\right)_{\mathcal{U}}=\left(\xi_{i}\right)_{\mathcal{U}}$ such that $\left\langle\xi_{i}^{\prime} \mid \eta_{i}\right\rangle=0$ for all $i \in I$ and $\left\|\xi_{i}^{\prime}\right\|=\left\|\left(\xi_{i}\right)\right\|_{\mathcal{U}}$ except on a null set. If each $\mathcal{H}_{i}$ is at least two-dimensional we can make the norm equality hold for all $i \in I$.

2. If $\left(\eta_{i}^{(1)}\right)_{\mathcal{U}}, \ldots,\left(\eta_{i}^{(d)}\right) \mathcal{U}$ form an orthonormal system in $\prod_{\mathcal{U}} \mathcal{H}_{i}$, then for $1 \leq j \leq d$ we can find representative sequences $\left(\xi_{i}^{(j)}\right)_{\mathcal{U}}=\left(\eta_{i}^{(j)}\right)_{\mathcal{U}}$ so that $\left\langle\xi_{i}^{(j)} \mid \xi_{i}^{(k)}\right\rangle=\delta_{j, k}$ for $\mathcal{U}$-many $i \in I$ and $1 \leq j, k \leq d$. If the $\mathcal{H}_{i}$ are at least $d$-dimensional we can do this for all $i \in I$.

In particular if $\left\{\left(\xi_{i}^{(j)}\right)_{i \rightarrow \mathcal{U}}\right\}_{j \in \mathbb{N}}$ is a countable orthonormal system in $\prod_{\mathcal{U}} \mathcal{H}_{i}$, where the $\mathcal{H}_{i}$ are infinite-dimensional, we can take representative sequences for each $i \in I$, $\left\{\xi_{i}^{(j)}\right\}_{j \in \mathbb{N}}$ is an orthonormal family. 
Proof. $\quad$ 1. Suppose $\xi=\left(\xi_{i}\right)_{\mathcal{U}}$, and $\eta=\left(\eta_{i}\right)_{\mathcal{U}}$ are orthogonal vectors in $\prod_{\mathcal{U}} \mathcal{H}_{i}$. Then

$$
\langle\xi \mid \eta\rangle_{\mathcal{U}}=\lim _{\mathcal{U}}\left\langle\xi_{i} \mid \eta_{i}\right\rangle_{i}=0
$$

If $\left(\eta_{i}\right)_{\mathcal{U}}=(0)_{\mathcal{U}}$ we are done. Otherwise there is a $\varepsilon>0$ so $S=\left\{i:\left\|\eta_{i}\right\| \geq \varepsilon\right\} \in$ $\mathcal{U}$. For $i \notin S$ let $\xi_{i}^{\prime}=0$, so $\left\langle\xi_{i}^{\prime} \mid \eta_{i}\right\rangle_{i}=0$. If each $\mathcal{H}_{i}$ is at least two-dimensional we can let $\xi_{i}^{\prime}$ be a unit vector orthogonal to $\eta_{i}$. For $i \in S$ let

$$
\xi_{i}^{\prime}=\xi_{i}-\frac{\left\langle\xi_{i} \mid \eta_{i}\right\rangle_{i}}{\left\|\eta_{i}\right\|^{2}} \eta_{i}
$$

Then

$$
\lim _{\mathcal{U}}\left\|\xi_{i}^{\prime}-\xi_{i}\right\|=\lim _{\mathcal{U}}\left\|\frac{\left\langle\xi_{i} \mid \eta_{i}\right\rangle}{\left\|\eta_{i}\right\|^{2}} \eta_{i}\right\| \leq \lim _{\mathcal{U}}\left|\left\langle\xi_{i} \mid \eta_{i}\right\rangle_{i}\right| \frac{1}{\left\|\eta_{i}\right\|}=0 .
$$

So we have that $\left(\xi_{i}\right)_{\mathcal{U}}=\left(\xi_{i}^{\prime}\right)_{\mathcal{U}}$, and for all $i \in S$,

$$
\left\langle\xi_{i}^{\prime} \mid \eta_{i}\right\rangle_{i}=\left\langle\xi_{i} \mid \eta_{i}\right\rangle_{i}-\frac{\left\langle\xi_{i} \mid \eta_{i}\right\rangle_{i}\left\langle\eta_{i} \mid \eta_{i}\right\rangle_{i}}{\|\left.\eta_{i}\right|^{2}}=0
$$

We can also scale the $\xi_{i}^{\prime}$ to have the same norm except on a null set. If each $\mathcal{H}_{i}$ is infinite-dimensional, we can ensure $\xi_{i}^{\prime}$ have the same norm for all $i$.

2. Without loss of generality we can assume $\left\|\eta_{j}^{(i)}\right\|=1$ for all $i \in I$ and $1 \leq j \leq d$. Let $\xi_{i}^{(1)}=\eta_{i}^{(1)}$ for all $i \in I$. By the previous part, we can find $\left(\xi_{i}^{(2)}\right) \mathcal{U}=\left(\eta_{i}^{(2)}\right)_{\mathcal{U}}$ so that $\left\langle\xi_{i}^{(2)} \mid \xi_{i}^{(1)}\right\rangle=0$ for all $i \in I$, and $\left\|\xi_{i}^{(2)}\right\|=1$ except on a null set. Then we can find $\left(\xi_{i}^{(3,1)}\right)_{\mathcal{U}}=\left(\eta_{i}^{(3)}\right) \mathcal{U}$ such that $\left\langle\xi_{i}^{(3,1)} \mid \xi_{i}^{(1)}\right\rangle=0$ for all $i$. Then we can find $\left(\xi_{i}^{(3)}\right) \mathcal{U}=\left(\xi_{i}^{(3,1)}\right)_{\mathcal{U}}$ such that $\left\langle\xi_{i}^{(3)} \mid \xi_{i}^{(2)}\right\rangle=0$ for all $i \in I$. Since $\xi_{i}^{(3)}-\xi_{i}^{(3,1)}=\frac{\left\langle\xi_{i}^{(3,1)} \mid \xi_{i}^{(2)}\right\rangle}{\left\|\xi_{i}^{(2)}\right\|} \xi_{i}^{(2)}$ is orthogonal to $\xi_{i}^{(1)}$ we have $\left\langle\xi_{i}^{(3)} \mid \xi_{i}^{(1)}\right\rangle=0$ for all $i \in I$ as well. The we can scale so $\left\|\xi_{i}^{(3)}\right\|=1$ up to a null set. By iterating this 
process we get the desired result. If each $\mathcal{H}_{i}$ is at least $d$-dimensional we can run this argument without worrying about null-sets.

\subsection{In $\mathrm{C}^{*}$-Algebras}

The following well-known results about nice representative sequences in $\mathrm{C}^{*}$-algebras will be very useful for us:

Proposition 3.2.1 (2.1 in [GH01], 5 and 17 in [BN09]). Let $\mathcal{U}$ be an ultrafilter on $I$, and for all $i \in I$ let $\mathfrak{A}_{i}$ be a non-trivial $\mathrm{C}^{*}$-algebra. Consider the ultraproduct $\prod_{\mathcal{U}} \mathfrak{A}_{i}$.

1. $\left(x_{i}\right)_{\mathcal{U}}$ is self-adjoint if and only if there is a representative sequence $\left(x_{i}\right)_{\mathcal{U}}=\left(t_{i}\right)_{\mathcal{U}}$ where each $t_{i}$ are self-adjoint.

2. $\left(x_{i}\right)_{\mathcal{U}}$ is positive if and only if there is a representative sequence $\left(x_{i}\right)_{\mathcal{U}}=\left(t_{i}\right)_{\mathcal{U}}$ where each of the $t_{i}$ are positive.

3. $\left(x_{i}\right)_{\mathcal{U}}$ is a projection if and only if there is a representative sequence $\left(x_{i}\right)_{\mathcal{U}}=\left(p_{i}\right)_{\mathcal{U}}$ where the $p_{i}$ are projections. In fact, if $p$, and $q$ are projections in $\prod_{\mathcal{U}} \mathfrak{A}_{i}$ with $q \leq p$, then for all $i$ there are projections $p_{i}$, and $q_{i} \in \mathfrak{A}_{i}$ with $q_{i} \leq p_{i}$ so that $p=\left(p_{i}\right)_{\mathcal{U}}$ and $q=\left(q_{i}\right)_{\mathcal{U}}$

4. $\left(x_{i}\right)_{\mathcal{U}}$ is a unitary if and only if there is a representative sequence $\left(x_{i}\right)_{\mathcal{U}}=\left(u_{i}\right)_{\mathcal{U}}$ where the $u_{i}$ are unitaries (potentially up to a null set on which the $\mathfrak{A}_{i}$ are not unital). 
5. If $p=\left(p_{i}\right)_{\mathcal{U}}$ and $q=\left(q_{i}\right)_{\mathcal{U}}$ are Murray-von Neumann equivalent projections, i.e. there is a partial isometry $v \in \prod_{\mathcal{U}} \mathfrak{A}_{i}$ with $v^{*} v=p$ and $v v^{*}=q$, and for all $i, p_{i}$ and $q_{i}$ are projections. Then there are partial isometries $v_{i}$ such that $v=\left(v_{i}\right)_{\mathcal{U}}$ and for $\mathcal{U}$-many $n, p_{i}=v_{i}^{*} v_{i}$ and $q_{i}=v_{i} v_{i}^{*}$.

For convenience and some flavor of the arguments we give some of the proofs.

Proof. 1. Suppose $\left(x_{i}\right)_{\mathcal{U}} \in \mathfrak{A}^{\mathcal{U}}$ is self-adjoint. Then $\left(x_{i}\right)_{\mathcal{U}}=\left(x_{i}\right)_{\mathcal{U}}^{*}=\left(x_{i}^{*}\right)$, so $\lim _{\mathcal{U}}\left\|x_{i}-x_{i}^{*}\right\|=0$. Recall we can represent

$$
x_{i}=\left(\frac{x_{i}+x_{i}^{*}}{2}\right)+i\left(\frac{x_{i}-x_{i}^{*}}{2 i}\right)=\Re\left(x_{i}\right)+i \Im\left(x_{i}\right),
$$

where $\Re\left(x_{i}\right)$ and $\Im\left(x_{i}\right)$ are self-adjoint. Then

$$
x_{i}^{*}=\left(\Re\left(x_{i}\right)+i \Im\left(x_{i}\right)\right)^{*}=\Re\left(x_{i}\right)-i \Im\left(x_{i}\right) .
$$

In particular, $x_{i}-x_{i}^{*}=2 i \Im\left(x_{i}\right)$. Thus since

$$
0=\lim _{\mathcal{U}}\left\|x_{i}-x_{i}^{*}\right\|=\lim _{U}\left\|2 i \Im\left(x_{i}\right)\right\|=2 \lim _{U}\left\|i \Im\left(x_{i}\right)\right\|,
$$

we have that $\left(i \Im\left(x_{i}\right)\right)_{\mathcal{U}}=(0)_{\mathcal{U}}$, so

$$
\left(x_{i}\right)_{\mathcal{U}}=\left(x_{i}\right)_{\mathcal{U}}-\left(i \Im\left(x_{i}\right)\right)_{\mathcal{U}}=\left(x_{i}-i \Im\left(x_{i}\right)\right)_{\mathcal{U}}=\left(\Re\left(x_{i}\right)\right)_{\mathcal{U}},
$$

where all the $\Re\left(x_{i}\right)$ are self-adjoint.

2. Suppose that $\left(x_{i}\right)_{\mathcal{U}} \in \prod_{\mathcal{U}} \mathfrak{A}_{i}$ is positive. Then there is a element $\left(y_{i}\right)_{\mathcal{U}} \in \prod_{\mathcal{U}} \mathfrak{A}_{i}$ so that $\left(x_{i}\right)_{\mathcal{U}}=\left(y_{i}\right)_{\mathcal{U}}^{*}\left(y_{i}\right)_{\mathcal{U}}=\left(y_{i}^{*} y_{i}\right)_{\mathcal{U}}$. 
3. Suppose $\left(x_{i}\right)_{\mathcal{U}} \in \prod_{\mathcal{U}} \mathfrak{A}_{i}$ is a projection. By the first part, we can assume the $x_{i}$ are self-adjoint. Since $\left(x_{i}\right)_{\mathcal{U}}$ is a projection, $\left(x_{i}\right)_{\mathcal{U}}=\left(x_{i}\right)_{\mathcal{U}}^{2}=\left(x_{i}^{2}\right)_{\mathcal{U}}$. Thus $\lim _{\mathcal{U}}\left\|x_{i}-x_{i}^{2}\right\|=0$. Consider the function $f(t)=t-t^{2}$ on $\sigma\left(x_{i}\right)$. By the functional calculus, $\lim _{\mathcal{U}}\left\|f\left(x_{i}\right)\right\|_{\infty}=0$. Thus the spectra of the $x_{i}$ are concentrating on $\{0,1\}$. In particular,

$$
S=\left\{i \mid \sigma\left(x_{i}\right) \subseteq[-1 / 3,1 / 3] \sqcup[2 / 3,4 / 3]\right\} \in \mathcal{U}
$$

For $i \notin S$ let $p_{i}=0$. Let $g(t)=1_{(1 / 2, \infty)}$ For $i \in S, g \in \mathcal{C}\left(\sigma\left(x_{i}\right)\right)$, so we can define $p_{i}=g\left(x_{i}\right)$, which are projections. Then

$$
\lim _{\mathcal{U}}\left\|x_{i}-p_{i}\right\|=\lim _{\mathcal{U}}\left\|f_{i}-g\right\|_{\infty, \sigma\left(x_{i}\right)}=0
$$

Thus $\left(x_{i}\right)_{\mathcal{U}}=\left(p_{i}\right)_{\mathcal{U}}$, where the $p_{i}$ are projections.

4. Suppose $\left(u_{i}\right)_{\mathcal{U}} \in \prod_{\mathcal{U}} \mathfrak{A}_{i}$ is a unitary. Then

$$
\lim _{\mathcal{U}}\left\|u_{i}^{*} u_{i}-I_{i}\right\|=0=\lim _{\mathcal{U}}\left\|u_{i} u_{i}^{*}-I_{i}\right\|
$$

Thus the set

$$
S=\left\{i:\left\|u_{i}^{*} u_{i}-I_{i}\right\| \text { and }\left\|u_{i} u_{i}^{*}-I_{i}\right\|<1\right\} \text { is in } \mathcal{U} \text {. }
$$

Thus for $i \in S, u_{i}^{*} u_{i}$ and $u_{i} u_{i}^{*}$ are invertible. So $u_{i}$ is both left- and rightinvertible, so it is invertible. Thus there is a polar decomposition $u_{i}=v_{i}\left|u_{i}\right|$ where $v_{i}$ is a unitary in $\mathfrak{A}_{i}$ and $\left|u_{i}\right|=\left(u_{i}^{*} u_{i}\right)^{1 / 2}$. For $i \notin S$ we just define $v_{i}=I_{i}$. Then $\lim _{\mathcal{U}}|| u_{i}-v_{i}\left\|=\lim _{\mathcal{U}}|| v_{i}\left|u_{i}\right|-v_{i} I_{i}||=\lim _{\mathcal{U}}|| v_{i}||\right\|\left|u_{i}\right|-I_{i} \|=0$. The 
last equality follows from the fact that $\lim _{\mathcal{U}}\left\|u_{i}^{*} u_{i}-I_{i}\right\|=0$ and the spectral calculus: if $\bar{\lambda} \lambda=|\lambda|^{2}$ is near one, then $|\lambda|$ is near one. Thus $\left(u_{i}\right)_{\mathcal{U}}=\left(v_{i}\right)_{\mathcal{U}}$.

In other words, we have that

1. $\left(\prod_{\mathcal{U}} \mathfrak{A}_{i}\right)_{s a}=\prod_{\mathcal{U}}\left(\mathfrak{A}_{i}\right)_{s a}$.

2. $\left(\prod_{\mathcal{U}} \mathfrak{A}_{i}\right)_{\geq 0}=\prod_{\mathcal{U}}\left(\mathfrak{A}_{i}\right)_{\geq 0}$.

3. $\mathcal{P}\left(\prod_{\mathcal{U}} \mathfrak{A}_{i}\right)=\prod_{\mathcal{U}} \mathcal{P}\left(\mathfrak{A}_{i}\right)$, where $\mathcal{P}(\mathfrak{A})$ is the set of projections in the $\mathrm{C}^{*}$-algebra $\mathfrak{A}$. This is even true even as partially ordered sets modulo Murray-von Neumann equivalence.

4. $U\left(\prod_{\mathcal{U}} \mathfrak{A}_{i}\right)=\prod_{\mathcal{U}} U\left(\mathfrak{A}_{i}\right)$.

We will see in Chapter 6 that normal elements do not always have representing sequences of normal elements, and we will see in Example 11.4.8 that central elements do not always have representing sequences of central elements. 


\section{Chapter 4}

\section{$\mathcal{K}\left(\mathcal{H}^{\mathcal{U}}\right), \mathcal{K}(\mathcal{H})^{\mathcal{U}}, \mathcal{B}(\mathcal{H})^{\mathcal{U}}$, and $\mathcal{B}\left(\mathcal{H}^{\mathcal{U}}\right)$}

We continue our quixotic quest to determine when parentheses and ultraproducts commute. We study the relationships between the four spaces $\mathcal{K}\left(\mathcal{H}^{\mathcal{U}}\right), \mathcal{K}(\mathcal{H})^{\mathcal{U}}$, $\mathcal{B}(\mathcal{H})^{\mathcal{U}}$, and $\mathcal{B}\left(\mathcal{H}^{\mathcal{U}}\right)$. Our starting point is the following result:

Proposition 4.0.2 (2.2 in [Hei80], Section 5 in [GH01]). There is a natural embedding of $\prod_{\mathcal{U}} \mathcal{B}\left(\mathcal{H}_{i}\right)$ into $\mathcal{B}\left(\prod_{\mathcal{U}} \mathcal{H}_{i}\right)$ by letting $\left(x_{i}\right)_{\mathcal{U}}$ act as the operator which sends a vector $\left(\xi_{i}\right)_{\mathcal{U}}$ to $\left(x_{i} \xi_{i}\right)_{\mathcal{U}}$. The norm of this operator is $\left\|\left(x_{i}\right)_{\mathcal{U}}\right\|=\lim _{\mathcal{U}}\left\|x_{i}\right\|$, so this embedding is isometric. In particular, for any Hilbert space $\mathcal{H},(\mathcal{B}(\mathcal{H}))^{\mathcal{U}}$ embeds into $\mathcal{B}\left(\mathcal{H}^{\mathcal{U}}\right)$.

Example 4.0.3. Fix $m \in \mathbb{N}$. If $\mathcal{U}$ is a free ultrafilter on $\mathbb{N}$ and for all $n \in \mathbb{N}$, $\mathcal{H}_{n}=\mathbb{C}^{m}$, since $\mathcal{B}\left(\mathcal{H}_{n}\right)$ is a finite-dimensional $\mathrm{C}^{*}$-algebra,

$$
\left(\mathcal{B}\left(\mathbb{C}^{m}\right)\right)^{\mathcal{U}} \cong\left(M_{m}\right)^{\mathcal{U}} \cong M_{m} \cong \mathcal{B}\left(\mathbb{C}^{m}\right) \cong \mathcal{B}\left(\left(\mathbb{C}^{m}\right)^{\mathcal{U}}\right)
$$


The situation is different when the Hilbert spaces are infinite-dimensional:

Example 4.0.4. Let $\mathcal{H} \cong \ell^{2}(\mathbb{N})$ is separable and infinite-dimensional, and $\mathcal{U}$ is a free ultrafilter on $\mathbb{N}$. Note that $\# \mathcal{B}(\mathcal{H})=\mathfrak{c}^{\operatorname{dim}(\mathcal{H})}$. Then we have

$$
\#(\mathcal{B}(\mathcal{H}))^{\mathcal{U}} \leq \# \ell^{\infty}(\mathcal{B}(\mathcal{H})) \leq\left(\mathfrak{c}^{\aleph_{0}}\right)^{\aleph_{0}}=\left(2^{\aleph_{0}}\right)^{\aleph_{0} \cdot \aleph_{0}}=\mathfrak{c}<2^{\mathfrak{c}}=\mathfrak{c}^{\mathfrak{c}}=\# \mathcal{B}\left(\mathcal{H}^{\mathcal{U}}\right)
$$

In particular, $(\mathcal{B}(\mathcal{H}))^{\mathcal{U}}$ is a proper subalgebra of $\mathcal{B}\left(\mathcal{H}^{\mathcal{U}}\right)$.

Projections in $\mathcal{B}\left(\mathcal{H}^{\mathcal{U}}\right)$ with countably infinite rank are not in the image of $\mathcal{B}(\mathcal{H})^{\mathcal{U}}$ :

Proposition 4.0.5. Let $\mathcal{U}$ be a free ultrafilter on $\mathbb{N}$. A projection $p=\left(p_{n}\right)_{\mathcal{U}} \in \mathcal{B}(\mathcal{H})^{\mathcal{U}}$, viewed as a projection in $\mathcal{B}\left(\mathcal{H}^{\mathcal{U}}\right)$, either has finite or uncountable rank. In particular, $p$ has rank $d$ if and only if $\left\{n \mid p_{n}\right.$ has rank $\left.d\right\} \in \mathcal{U}$.

Proof. The argument is very similar to the proof of Proposition 2.6.2.

Suppose $R_{d}=\left\{n \mid p_{n}\right.$ has rank $\left.d\right\} \in \mathcal{U}$. Then $\left(p_{n}\right)_{\mathcal{U}} \mathcal{H}^{\mathcal{U}}=\prod_{\mathcal{U}} p_{n} \mathcal{H}$ is $d$-dimensional by 2.6 .2 .

Conversely, suppose for all $d \in \mathbb{N},\left\{n \mid p_{n}\right.$ has rank $\left.d\right\} \notin \mathcal{U}$. Then for all $d$,

$$
G_{d}=\left\{n \mid p_{n} \text { has rank at least } d\right\} \text { is in } \mathcal{U}
$$

For $n \in \mathbb{N}$, define $K_{n}=\min \left(n, \operatorname{rank}\left(p_{n}\right)\right)$, and let $\xi_{n}^{(1)}, \ldots, \xi_{n}^{\left(K_{n}\right)}$ be an orthonormal set in $p_{n}\left(\mathcal{H}_{n}\right)$. For $0 \leq r<1$, for each $n \in \mathbb{N}$ there is a unique integer $r_{n}, 1 \leq r_{n} \leq K_{n}$ so that $\frac{r_{n}-1}{K_{n}} \leq r<\frac{r_{n}}{K_{n}}$. Define $\Phi:[0,1) \rightarrow \prod_{u} \mathcal{H}_{n}$ by $\Phi(r)=\left(\xi_{n}^{\left(r_{n}\right)}\right)_{\mathcal{U}}$. We claim that $\{\Phi(r)\}_{0 \leq r<1}$ is orthonormal. Clearly $\|\Phi(r)\|=\lim _{\mathcal{U}}\left\|\xi_{n}^{\left(r_{n}\right)}\right\|=1$. If $0 \leq r, s<1$, $r \neq s$, there is an $N \in \mathbb{N}$ so that $|r-s|>1 / N$. Thus for all $n$ with $K_{n} \geq N, r_{n} \neq s_{n}$ 
since they are in different elements in the partition of $[0,1)$ into $K_{n}$ subintervals of length $1 / K_{n}<1 / N$. Note that the set $S=\left\{n \mid K_{n} \geq N\right\}=\{n \mid n \geq N\} \cap G_{N} \in \mathcal{U}$. Then for $n \in S,\left\langle\xi_{n}^{\left(r_{n}\right)} \mid \xi_{n}^{\left(s_{n}\right)}\right\rangle=0$. Thus $\langle\Phi(r) \mid \Phi(s)\rangle=\lim _{\mathcal{U}}\left\langle\xi_{n}^{\left(r_{n}\right)} \mid \xi_{n}^{\left(s_{n}\right)}\right\rangle=0$. So $\{\Phi(r)\}_{0 \leq r<1}$ is an uncountable orthonormal system in $\left(p_{n}\right)_{\mathcal{U}} \prod_{\mathcal{U}} \mathcal{H}_{n}$

Question 4.0.6. Can we identify the images of $\mathcal{K}(\mathcal{H})^{\mathcal{U}}$ and $\mathcal{B}(\mathcal{H})^{\mathcal{U}}$ in $\mathcal{B}\left(\mathcal{H}^{\mathcal{U}}\right)^{\text {? }}$

The following result is an extension of Lemma 5.3 in [GH01], where it was observed that $\mathcal{K}\left(\prod_{\mathcal{U}} \mathcal{H}_{i}\right)$ was contained in the image of $\prod_{\mathcal{U}} \mathcal{K}\left(\mathcal{H}_{i}\right)$ in $\mathcal{B}\left(\prod_{\mathcal{U}} \mathcal{H}_{i}\right)$ since the rank-one operators in $\mathcal{B}\left(\prod_{\mathcal{U}} \mathcal{H}_{i}\right)$ are in the image.

\section{Proposition 4.0.7.}

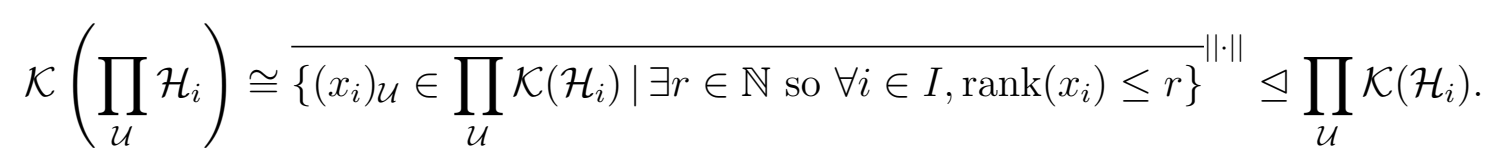

Proof. For vectors $\xi, \eta$ in a Hilbert space $\mathcal{H}$, let $t_{\xi, \eta}$ be the rank-one operator defined by $t_{\xi, \eta}(\psi)=\langle\psi \mid \xi\rangle \eta$. Then any rank-one operator $T$ is of the form $\alpha t_{\xi, \eta}$. Then a finite-rank operator $T$ in $\mathcal{K}\left(\prod_{\mathcal{U}} \mathcal{H}_{n}\right)$ can be written as

$$
T=\sum_{j=1}^{r} \alpha_{j} t_{\xi^{(j)}, \eta^{(j)}}
$$

where $\xi^{(j)}, \eta^{(j)} \in \mathcal{H}^{\mathcal{U}}$ so $\xi=\left(\xi_{i}^{(j)}\right) \mathcal{U}, \eta=\left(\eta_{i}^{(j)}\right)_{\mathcal{U}}$. Then we define

$$
\left.\Psi(T)=\left(\sum_{j=1}^{m} \alpha_{j} t_{\xi_{i}^{(j)}, \eta_{i}^{(j)}}\right)\right)_{\mathcal{U}}
$$

Note that $\Psi$ is the inverse of the map that embeds $\prod_{\mathcal{U}} \mathcal{B}\left(\mathcal{H}_{i}\right)$ into $\mathcal{B}\left(\prod_{\mathcal{U}} \mathcal{H}_{i}\right)$. In particular $\Psi$ is a well-defined $*$-homomorphism. Thus $\Psi$ extends to $*$-isomorphism from 
$\mathcal{K}\left(\prod_{\mathcal{U}} \mathcal{H}_{i}\right)$ onto its image, which is the norm-closure of the elements in $\prod_{\mathcal{U}} \mathcal{K}\left(\mathcal{H}_{i}\right)$ which have a representation with uniformly bounded rank.

Question 4.0.8. If $\mathcal{H}$ is infinite-dimensional and separable, is

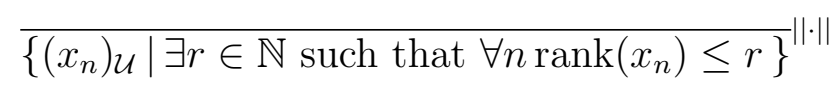

the maximal CCR ideal of $\mathcal{K}(\mathcal{H})^{\mathcal{U}}$ ?

If we let $\mathfrak{A}$ be the image of $\mathcal{B}(\mathcal{H})^{\mathcal{U}}$ in $\mathcal{B}\left(\mathcal{H}^{\mathcal{U}}\right)$, we have

Corollary 4.0.9.

$$
\mathfrak{A} / \mathcal{K}\left(\mathcal{H}^{\mathcal{U}}\right) \cong \mathcal{B}(\mathcal{H})^{\mathcal{U}} / \overline{\left\{\left(x_{n}\right)_{\mathcal{U}} \mid \exists r \in \mathbb{N} \text { such that } \forall n \operatorname{rank}\left(x_{n}\right) \leq r\right\}}|| \cdot \|
$$

Proposition 4.0.10 (5.1 in [GH01]). Let $\mathcal{U}$ be an ultrafilter on $I$, and for all $i \in I$ suppose $\mathfrak{A}_{i}$ and $\mathfrak{B}_{i}$ are $\mathrm{C}^{*}$-algebras and $\phi_{i}: \mathfrak{A}_{i} \rightarrow \mathfrak{B}_{i}$ is a $*$-homomorphism. Then there is a $*$-homomorphism $\Phi: \prod_{\mathcal{U}} \mathfrak{A}_{i} \rightarrow \prod_{\mathcal{U}} \mathfrak{B}_{i}$ defined by $\Phi\left(\left(x_{i}\right)_{\mathcal{U}}\right)=\left(\phi_{i}\left(x_{i}\right)\right)_{\mathcal{U}}$ and $\operatorname{ker}(\Phi)=\prod_{\mathcal{U}} \operatorname{ker}\left(\phi_{i}\right)$

In particular, if $J_{i}$ are closed, two-sided ideals in $\mathfrak{A}_{i}$, then $\prod_{\mathcal{U}}\left(\mathfrak{A}_{i}\right) / \prod_{\mathcal{U}}\left(J_{i}\right) \cong$ $\prod_{\mathcal{U}}\left(\mathfrak{A}_{i} / J_{i}\right)$. Thus ultraproducts preserve exact sequences.

Example 4.0.11. If $\mathfrak{A}$ is a non-unital $\mathrm{C}^{*}$-algebra, then we denote its unitization by $\mathfrak{A}^{\sim}$. Then we see $\left(\prod_{\mathcal{U}} \mathfrak{A}_{n}\right)^{\sim} \cong \prod_{\mathcal{U}} \mathfrak{A}_{n}^{\sim}$, since the $\mathrm{C}^{*}$-algebra on the right hand side is clearly unital, and $\prod_{\mathcal{U}} \mathfrak{A}_{n}^{\sim} / \prod_{\mathcal{U}} \mathfrak{A}_{n} \cong \prod_{\mathcal{U}}\left(\mathfrak{A}_{n}^{\sim} / \mathfrak{A}_{n}\right) \cong \prod_{\mathcal{U}} \mathbb{C} \cong \mathbb{C}$.

Example 4.0.12. $(\mathcal{B}(\mathcal{H}))^{\mathcal{U}} /(\mathcal{K}(\mathcal{H}))^{\mathcal{U}} \cong(B(\mathcal{H}) / K(\mathcal{H}))^{\mathcal{U}}$. 
In general ultraproducts of simple $\mathrm{C}^{*}$-algebra are not simple (see e.g. 7.3.3), but this one is:

Proposition 4.0.13 (5.6 in [GH01]). Suppose for all $i \in I, \mathfrak{A}_{i}$ is a unital C*-algebra and there is an $N \in \mathbb{N}$ and $M \geq 0$ with the following property: for all $a_{i} \in \mathfrak{A}_{i}$ with $\left\|a_{i}\right\|=1$ there are elements $b^{(j)}, c^{(j)}, 1 \leq j \leq N$ of norm no more than $M$ so that $I_{i}=\sum_{j=1}^{N} b_{j} a_{i} c_{j}$. Then $\prod_{\mathcal{U}} \mathfrak{A}_{i}$ is simple.

Corollary 4.0.14 (5.7 in [GH01]). All of the ultrapowers of the Calkin algebra $\mathcal{B}(\mathcal{H}) / \mathcal{K}(\mathcal{H})$ are simple.

In conclusion, we have shown the following relations:

$$
\mathcal{K}\left(\mathcal{H}^{\mathcal{U}}\right) \cong{\overline{\left\{\left(x_{n}\right)_{\mathcal{U}} \mid \exists r \in \mathbb{N} \text { such that } \forall n \operatorname{rank}\left(x_{n}\right) \leq r\right.}}^{\|\cdot\|} \unlhd \mathcal{K}(\mathcal{H})^{\mathcal{U}} \unlhd \mathcal{B}(\mathcal{H})^{\mathcal{U}} \hookrightarrow \mathcal{B}\left(\mathcal{H}^{\mathcal{U}}\right)
$$

All of the containments are proper when $\mathcal{H}$ is infinite-dimensional. 


\section{Chapter 5}

\section{Spectra in Ultraproducts}

We study how the spectrum of an element $\left(a_{i}\right)_{\mathcal{U}}$ in an ultraproduct is related to the spectra of the $a_{i}$. For more information about continuity of spectra see [New51], [CM79], [CM81], [CM83], as well as Chapter 14 in [AFHV84].

\subsection{Preliminaries}

Lemma 5.1.1 (100 in [Hal82]). The set of invertible operators is open and inversion is a continuous function on this set.

Definition 5.1.2. Let $A_{n} \subseteq \mathbb{C}$. We define the limit superior of the $A_{n}$ to be $\underset{n}{\limsup } A_{n}=\left\{\lambda \in \mathbb{C}:\right.$ there is a sequence $\lambda_{n} \in A_{n}$ such that $\lambda$ is a limit point of $\left.\left(\lambda_{n}\right)\right\}$. And similarly we define the limit inferior of the $A_{n}$ to be $\liminf _{n} A_{n}=\left\{\lambda \in \mathbb{C}:\right.$ there is a convergent sequence $\left.\lambda_{n} \in A_{n}, \lim _{n} \lambda_{n}=\lambda\right\}$ 
Clearly $\liminf { }_{n} A_{n} \subseteq \lim \sup _{n} A_{n}$. We say a sequence of sets $A_{n} \subseteq \mathbb{C}$ is convergent if

$$
\liminf _{n} A_{n}=A=\limsup _{n} A_{n}
$$

in which case we write $\lim _{n} A_{n}=A$.

Lemma 5.1.3 (Upper Semicontinuity of Spectrum, 103 in [Hal82]). If $a_{n} \stackrel{\|\cdot\|}{\longrightarrow} a$ in a $\mathrm{C}^{*}$-algebra $\mathfrak{A}$, then $\limsup _{n} \sigma\left(a_{n}\right) \subseteq \sigma(a)$. This is known as sequential upper semicontinuity.

Equivalently, for all $U^{\text {open }} \supseteq \sigma(a)$, there is a $\varepsilon>0$ such that if $\|a-x\|<\varepsilon$, then $\sigma(x) \subseteq U$. This is known as metric upper semicontinuity.

We say spectrum is continuous at an operator $a$ if $a_{n} \stackrel{\|\cdot\|}{\longrightarrow} a$ implies that $\sigma\left(a_{n}\right) \rightarrow$ $\sigma(a)$ as defined above. Since upper semicontinuity has been established, continuity of spectra at $a$ depends on whether

$$
\sigma(a) \subseteq \liminf _{n} \sigma\left(a_{n}\right)
$$

for all $a_{n} \stackrel{\|\cdot\|}{\longrightarrow} a$.

Here are some general results about points of continuity of the spectrum.

Proposition 5.1.4 ([New51]). If $a$ is a compact operator, or, more generally, if $\sigma(a)$ is totally disconnected, then $a$ is a point of continuity of spectrum. Spectrum is continuous on commutative $\mathrm{C}^{*}$-algebras.

Proposition 5.1.5 (105 in [Hal82]). The operation of taking the spectrum is continuous on the set of normal operators. 


\subsection{Pseudospectra}

In a rare example of serendipitous terminology, the notion of pseudospectrum is relevant to our research. See e.g. [Tre92], [Tre97], [TE05], and [TT94].

Definition 5.2.1. Let $\varepsilon>0$. The $\varepsilon$-pseudospectrum of an element $x$ in a $\mathrm{C}^{*}$-algebra $\mathfrak{A}$ is

$$
\sigma_{\varepsilon}(x):=\left\{\lambda \in \mathbb{C}: \lambda \in \sigma(x) \text { or }\left\|(x-\lambda I)^{-1}\right\|>\frac{1}{\varepsilon}\right\}
$$

Equivalently,

$$
\sigma_{\varepsilon}(x):=\{\lambda \in \sigma(x+e): \text { for some } e \text { with }\|e\|<\varepsilon\}=\bigcup_{\|e\|<\varepsilon} \sigma(x+e) .
$$

In other words, the $\varepsilon$-pseudospectrum is the open subset of the plane bounded by the $1 / \varepsilon$ level curve of the norm of the resolvent.

The $\varepsilon$-pseudospectra are nested: if $0<\varepsilon_{2}<\varepsilon_{1}$ then

$$
\sigma(x) \subseteq \sigma_{\varepsilon_{2}}(x) \subseteq \sigma_{\varepsilon_{1}}(x)
$$

The intersection of all the pseusospectra is the spectrum:

$$
\bigcap_{n \in \mathbb{N}} \sigma_{1 / n}(x)=\sigma(x)
$$

Lemma 5.2.2. Suppose $x$ is an invertible operator and $y$ is an operator such that $\|I-y x\|<\varepsilon<1$. Then $\left\|x^{-1}\right\| \leq\|y\| /(1-\varepsilon)$.

Proof.

$$
\left\|x^{-1}\right\|=\left\|(I-y x+y x) x^{-1}\right\| \leq\left\|(I-y x) x^{-1}\right\|+\|y\| \leq \varepsilon\left\|x^{-1}\right\|+\|y\| .
$$


Thus $\left\|x^{-1}\right\| \leq \frac{\|y\|}{1-\varepsilon}$ as claimed.

The following is a special case of Theorem 1 in [BN11]:

Proposition 5.2.3. An element $\left(x_{i}\right)_{\mathcal{U}} \in \prod_{\mathcal{U}} \mathfrak{A}_{i}$ is invertible if and only if there exists an $M \in \mathbb{R}$ so that $\mathcal{I}_{M}=\left\{i \mid x_{i}\right.$ is invertible and $\left.\left\|x_{i}^{-1}\right\|<M\right\} \in \mathcal{U}$.

Proof. Suppose there is an $M$ so $\mathcal{I}_{M}=\left\{i \mid x_{i}\right.$ is invertible and $\left.\left\|x_{i}^{-1}\right\|<M\right\} \in \mathcal{U}$.

Then let

$$
y_{i}= \begin{cases}x_{i}^{-1}, & \text { if } i \in \mathcal{I}_{M} \\ 0, & \text { otherwise }\end{cases}
$$

Then $\left\|\left(y_{i}\right)_{\mathcal{U}}\right\| \leq M$ and

$$
\left(x_{i}\right)_{\mathcal{U}}\left(y_{i}\right)_{\mathcal{U}}=\left(I_{i}\right)_{\mathcal{U}}=\left(y_{i}\right)_{\mathcal{U}}\left(x_{i}\right)_{\mathcal{U}}
$$

Thus $\left(x_{i}\right)_{\mathcal{U}}$ is invertible.

Conversely, if $\left(x_{i}\right)_{\mathcal{U}}$ is invertible, then $\prod_{\mathcal{U}} \mathfrak{A}_{i}$ is unital so $\mathcal{U}$-many $A_{i}$ are unital (see Theorem 8.3.2), so we can assume the unit of $\prod_{\mathcal{U}} \mathfrak{A}_{i}$ is $\left(I_{i}\right)_{\mathcal{U}}$ where $I_{i}$ is the unit of $\mathfrak{A}_{i}$. Thus if $\left(x_{i}\right)_{\mathcal{U}}$ is invertible, then there is a sequence $\left(y_{i}\right)_{\mathcal{U}}$ so that

$$
\left(x_{i}\right)_{\mathcal{U}}\left(y_{i}\right)_{\mathcal{U}}=\left(I_{i}\right)_{\mathcal{U}}=\left(y_{i}\right)_{\mathcal{U}}\left(x_{i}\right)_{\mathcal{U}}
$$

Thus

$$
\lim _{\mathcal{U}}\left\|x_{i} y_{i}-I\right\|=0=\lim _{\mathcal{U}}\left\|y_{i} x_{i}-I\right\|
$$

Recall that if $\|z-I\|<1$ then $z$ is invertible. Thus there is an $U_{1} \in \mathcal{U}$ so that for $i \in U_{1},\left\|x_{i} y_{i}-I\right\|<\frac{1}{2}$. Similarly there is an $U_{2} \in \mathcal{U}$ so that for $i \in U_{2},\left\|y_{i} x_{i}-I\right\|<\frac{1}{2}$. 
Then for $i \in U=U_{1} \cap U_{2} \in \mathcal{U}, x_{i} y_{i}$ and $y_{i} x_{i}$ are invertible, which means that $x_{i}$ is invertible. By the previous lemma, for $i \in U$,

$$
\left\|x_{i}^{-1}\right\| \leq 2|| y_{i} \|
$$

Thus since the $y_{i}$ are uniformly bounded, so are the $x_{i}^{-1}$. Thus $\left(x_{i}^{-1}\right)_{\mathcal{U}}$ is well-defined, and in fact, $\left(x_{i}^{-1}\right)_{\mathcal{U}}=\left(y_{i}\right)_{\mathcal{U}}$ by the uniqueness of inverses.

Thus $\lambda \notin \sigma\left(x_{i}\right)_{\mathcal{U}}$ if and only if $\left(x_{i}-\lambda I\right)_{\mathcal{U}}$ is invertible if and only if there is an $M$ so $\left\{i \mid x_{i}\right.$ is invertible and $\left.\left\|x_{i}^{-1}\right\|<M\right\} \in \mathcal{U}$ if and only if there is an $M$ so that $\left\{i \mid \lambda \notin \sigma_{1 / M}(x)\right\} \in \mathcal{U}$.

\subsection{Ultralimits of Sets}

To study the spectrum of elements in ultraproducts, we introduce the following notion:

Definition 5.3.1. Let $\mathcal{U}$ be an ultrafilter on $I$ and for all $i \in I$ suppose $A_{i} \subseteq \mathbb{C}$. We define the ultralimit of the $A_{i}$ with respect to $\mathcal{U}$ to be

$$
\lim _{\mathcal{U}} A_{i}:=\left\{\lim _{\mathcal{U}} \lambda_{i} \mid \lambda_{i} \in A_{i} \text { and } \sup _{i} \| \lambda_{i}||<\infty\right\}
$$

It is okay if $A_{i}=\emptyset$ for a small set of indices, or $\lambda_{i} \notin A_{i}$ on a small set of indices, or $\lambda_{i}$ is undefined on a small set of indices.

Proposition 5.3.2. If $\mathcal{U}$ is an ultrafilter on $I$, and for all $i \in I A_{i} \subseteq B_{i}$ are subsets of $\mathbb{C}$, then $\lim _{\mathcal{U}} A_{i} \subseteq \lim _{\mathcal{U}} B_{i}$. 
Proof. This is clear, if $\lambda \in \lim _{\mathcal{U}} A_{i}$ there are $\lambda_{i} \in A_{i} \subseteq B_{i}$ so $\lim _{\mathcal{U}} \lambda_{i}=\lambda$, so $\lambda \in \lim _{\mathcal{U}} B_{i}$

Proposition 5.3.3. If $\mathcal{U}$ is an ultrafilter on $I$, and for all $i \in I, A_{i}$ and $B_{i}$ are subsets of $\mathbb{C}$, then $\lim _{\mathcal{U}}\left(A_{i} \cup B_{i}\right)=\left(\lim _{\mathcal{U}} A_{i}\right) \cup\left(\lim _{\mathcal{U}} B_{i}\right)$.

Proof. Since $A_{i}, B_{i} \subseteq A_{i} \cup B_{i}$, it is clear that $\left(\lim _{\mathcal{U}} A_{i}\right) \cup\left(\lim _{\mathcal{U}} B_{i}\right) \subseteq \lim _{\mathcal{U}}\left(A_{i} \cup B_{i}\right)$. Conversely, if $\lambda \in \lim _{\mathcal{U}}\left(A_{i} \cup B_{i}\right)$ then there are $\lambda_{i} \in A_{i} \cup B_{i}$ so that $\lim _{\mathcal{U}} \lambda_{i}=\lambda$, and

$$
I=\left\{i \mid \lambda_{i} \in A_{i} \backslash B_{i}\right\} \sqcup\left\{i \mid \lambda_{i} \in B_{i}\right\}
$$

Thus either $\lambda_{i} \in A_{i} \mathcal{U}$-almost everywhere, in which case $\lambda \in \lim _{\mathcal{U}} A_{i}$, or $\lambda_{i} \in B_{i}$ $\mathcal{U}$-almost everywhere, in which case $\lambda \in \lim _{\mathcal{U}} B_{i}$.

Proposition 5.3.4. If $\mathcal{U}$ is an ultrafilter on $I$, and for all $i \in I, A_{i}$ and $B_{i}$ are subsets of $\mathbb{C}$, then $\lim _{\mathcal{U}}\left(A_{i} \cap B_{i}\right) \subseteq\left(\lim _{\mathcal{U}} A_{i}\right) \cap\left(\lim _{\mathcal{U}} B_{i}\right)$

Proof. This is clear since $A_{i} \cap B_{i}$ is a subset of both $A_{i}$ and $B_{i}$.

Proposition 5.3.5. If $\mathcal{U}$ is an ultrafilter on $I$, and for all $i \in I, A_{i}$ and $B_{i}$ are subsets of $\mathbb{C}$, then $\lim _{\mathcal{U}}\left(A_{i} \backslash B_{i}\right) \supseteq\left(\lim _{\mathcal{U}} A_{i}\right) \backslash\left(\lim _{\mathcal{U}} B_{i}\right)$.

Proof. If $\lambda \in\left(\lim _{\mathcal{U}} A_{i}\right) \backslash\left(\lim _{\mathcal{U}} B_{i}\right)$, then there are $\lambda_{i} \in A_{i}$ with $\lim _{\mathcal{U}} \lambda_{i}=\lambda$, and for all $\mu_{i} \in B_{i}$, we have $\lim _{\mathcal{U}} \mu_{i} \neq \lambda$. Thus on a large set, $\lambda_{i} \in A_{i} \backslash B_{i}$. So $\lambda \in$ $\lim _{\mathcal{U}}\left(A_{i} \backslash B_{i}\right)$ 
In particular, $\lim _{\mathcal{U}}\left(\mathbb{C} \backslash A_{i}\right) \supseteq \mathbb{C} \backslash\left(\lim _{\mathcal{U}} A_{i}\right)$, and

$$
\mathbb{C} \backslash\left[\lim _{\mathcal{U}}\left[\mathbb{C} \backslash A_{i}\right]\right] \subseteq \lim _{\mathcal{U}} A_{i}
$$

One might consider the left-hand side as a sort of ultra-limit infimum or ultra-interior of the $A_{i}$.

The reverse containments can fail, for instance if $A_{n}=[-1,-1 / n]$ and $B_{n}=$ $[1 / n, 1]$, and $\mathcal{U}$ is an ultrafilter on $\mathbb{N}$, then $\left(\lim _{\mathcal{U}} A_{n}\right) \cap\left(\lim _{\mathcal{U}} B_{n}\right)=[-1,0] \cap[0,1]=\{0\}$ but $\lim _{\mathcal{U}}\left(A_{n} \cap B_{n}\right)=\lim _{\mathcal{U}} \emptyset=\emptyset$.

Recall the Hausdorff distance between bounded sets $A$ and $B$ is defined by

$$
\begin{aligned}
\operatorname{dist}(A, B) & =\max \left(\sup _{a \in A} \operatorname{dist}(a, B), \sup _{b \in B} \operatorname{dist}(b, A)\right) \\
& =\max \left(\sup _{a \in A} \inf _{b \in B} \operatorname{dist}(a, b), \sup _{b \in B} \inf _{a \in A} \operatorname{dist}(a, b)\right) .
\end{aligned}
$$

To allow for unbounded sets we instead use

$$
\operatorname{dist}^{\prime}(A, B)=\min (\operatorname{dist}(A, B), 1)
$$

Recall for $\varepsilon \geq 0$ the $\varepsilon$-fattening of $A$ is

$$
A_{\varepsilon}=\{\lambda \in \mathbb{C}: \operatorname{dist}(\lambda, A) \leq \varepsilon\}
$$

If we take any sequence $\varepsilon_{i}>0$ with $\lim _{\mathcal{U}} \varepsilon_{i}=0$, we have that

$$
\lim _{\mathcal{U}} A_{i}=\lim _{\mathcal{U}}\left(A_{i}\right)_{\varepsilon_{i}}
$$

In fact, 
Proposition 5.3.6. $\lim _{\mathcal{U}} A_{i}=\lim _{\mathcal{U}} B_{i}$ if and only if $\lim _{\mathcal{U}} \operatorname{dist}^{\prime}\left(A_{i}, B_{i}\right)=0$.

Proof. Suppose $\lim _{\mathcal{U}} \operatorname{dist}^{\prime}\left(A_{i}, B_{i}\right)=0$, then if $\lambda \in \lim _{\mathcal{U}} A_{i}$ with $\lim _{\mathcal{U}} \lambda_{i}=\lambda$, for all $i$ there is a $\mu_{i} \in B_{i}$ with $\operatorname{dist}\left(\lambda_{i}, \mu_{i}\right)<2 \operatorname{dist}^{\prime}\left(A_{i}, B_{i}\right)$, so if $e_{i}=\lambda_{i}-\mu_{i}$, we have

$$
\lim _{\mathcal{U}}\left|e_{i}\right| \leq \lim _{\mathcal{U}} \operatorname{dist}^{\prime}\left(A_{i}, B_{i}\right)=0
$$

Thus $\lim _{\mathcal{U}} \mu_{i}=\lim _{\mathcal{U}} \lambda_{i}-e_{i}=\lim _{\mathcal{U}} \lambda_{i}=\lambda$, so $\lambda \in \lim _{\mathcal{U}} B_{i}$.

Conversely, if $\lim _{\mathcal{U}} \operatorname{dist}^{\prime}\left(A_{i}, B_{i}\right)=\varepsilon>0$, then we can find a sequence $x_{i}$, so for all $i, x_{i}=a_{i} \in A_{i}$ and $\operatorname{dist}\left(a_{i}, B_{i}\right) \geq \varepsilon / 2$, or $x_{i}=b_{i} \in B_{i}$ and $\operatorname{dist}\left(b_{i}, A_{i}\right) \geq \varepsilon / 2$. Either $\left\{i \mid x_{i}=a_{i}\right\} \in \mathcal{U}$, or $\left\{i \mid x_{i}=b_{i}\right\} \in \mathcal{U}$. Suppose $\left\{i \mid x_{i}=a_{i}\right\} \in \mathcal{U}$. So $\operatorname{dist}\left(a_{i}, B_{i}\right) \geq \varepsilon / 2$. Let $\lambda=\lim _{\mathcal{U}} \lambda_{i}$, then $\lambda \notin \lim _{\mathcal{U}} B_{i}$.

Proposition 5.3.7. Let $\mathcal{U}$ be an ultrafilter on $I$, and for all $i \in I$ let $\mathfrak{A}_{i}$ be a $\mathrm{C}^{*}$ algebra. If for all $i \in I, x_{i} \in \mathfrak{A}_{i}$ and $\sup _{i}\left\|x_{i}\right\|<\infty$, then $\lim _{\mathcal{U}} \sigma\left(x_{i}\right)$ is compact.

Proof. This is trivial if $\mathcal{U}$ is principal, so we will assume $\mathcal{U}$ is free. Note that $\sigma\left(x_{i}\right) \subseteq$ $\mathcal{B}\left(0,\left\|x_{i}\right\|\right) \subseteq \mathcal{B}\left(0, \sup _{i}\left\|x_{i}\right\|\right)$, so $\lim _{\mathcal{U}} \sigma\left(x_{i}\right)$ is bounded. Suppose $\lambda^{(k)} \in \lim _{\mathcal{U}} \sigma\left(x_{i}\right)$ so that $\lim _{k \rightarrow \infty} \lambda^{(k)}=\lambda$. By taking a subsequence we can assume $\left|\lambda^{(k)}-\lambda\right|<1 / k$ for all $k \in \mathbb{N}$. Then for each $k$ there are $\lambda_{i}^{(k)} \in \sigma\left(x_{i}\right)$ such that $\lim _{i \rightarrow \mathcal{U}} \lambda_{i}^{(k)}=\lambda^{(k)}$. Let

$$
A^{(k)}=\left\{i:\left|\lambda_{i}^{(k)}-\lambda^{(k)}\right|<\frac{1}{k}\right\} \in \mathcal{U}
$$

Then let $B_{1}=A^{(1)} \subseteq\left\{i:\left|\lambda_{n}^{(1)}-\lambda\right|<2\right\}$, and for $m>1$,

$$
B_{m}=\bigcap_{1 \leq k \leq m} A^{(k)} \subseteq\left\{i:\left|\lambda_{i}^{(m)}-\lambda\right|<\frac{2}{m} \text { for } 1 \leq j \leq m\right\} .
$$


Then $B_{m} \in \mathcal{U}$ and $B_{m} \supseteq B_{m+1}$ for all $m$. For $i \notin B_{1}$ pick $\mu_{i} \in \sigma\left(x_{i}\right)$ however you want. For $i \in B_{m} \backslash B_{m+1}$ let $\mu_{i}=\lambda_{i}^{(m)}$ and note that $\left|\mu_{i}-\lambda\right|<2 / m$. For $i \in \bigcap_{m} B_{m}$, we must have $\lambda \in \sigma\left(x_{i}\right)$, so we let $\mu_{i}=\lambda$. The we have for all $i \in B_{m} \in \mathcal{U}$, $\left|\mu_{i}-\lambda\right|<2 / m$. Thus $\lim _{\mathcal{U}} \mu_{i}=\lambda$.

\subsection{Spectra in Ultraproducts}

Proposition 5.4.1.

$$
\sigma\left(\left(x_{i}\right)_{\mathcal{U}}\right) \subseteq \bigcap_{n \in \mathbb{N}} \lim _{\mathcal{U}} \sigma_{1 / n}\left(x_{i}\right)
$$

Proof. Suppose $\lambda \in \sigma\left(\left(x_{i}\right)_{\mathcal{U}}\right)$, so $\left(x_{i}-\lambda I\right)_{\mathcal{U}}$ is not invertible, so either $\left\{i \mid x_{i}-\right.$ $\lambda I$ not inv. $\} \in \mathcal{U}$, or $\left\{i \mid x_{i}-\lambda I\right.$ inv. $\} \in \mathcal{U}$ but $\lim _{\mathcal{U}}\left\|\left(x_{i}-\lambda I\right)^{-1}\right\|=\infty$.

If $\left\{i \mid x_{i}-\lambda I\right.$ not inv. $\} \in \mathcal{U}$, then $\left\{i \mid \lambda \in \sigma\left(x_{i}\right)_{\mathcal{U}}\right\} \in \mathcal{U}$ so $\lambda \in \lim _{\mathcal{U}} \sigma\left(x_{i}\right) \subseteq$ $\lim _{\mathcal{U}} \sigma_{1 / n}\left(x_{i}\right)$ for all $n$. Thus $\lambda \in \bigcap_{n \in \mathbb{N}} \lim _{\mathcal{U}} \sigma_{1 / n}\left(x_{i}\right)$.

If $\left\{i \mid x_{i}-\lambda I\right.$ not inv. $\} \in \mathcal{U}$, but $\lim _{\mathcal{U}}\left\|\left(x_{i}-\lambda I\right)^{-1}\right\|=\infty$. So for all $n,\left\{i \mid \|\left(x_{i}-\right.\right.$ $\left.\lambda I)^{-1}>n\right\} \in \mathcal{U}$. So $\left\{i \mid \lambda \in \sigma_{1 / n}\left(x_{i}\right)\right\} \in \mathcal{U}$. Thus $\lambda \in \bigcap_{n \in \mathbb{N}} \lim _{\mathcal{U}} \sigma_{1 / n}\left(x_{i}\right)$.

\section{Proposition 5.4.2.}

$$
\bigcap_{n \in \mathbb{N}}\left[\mathbb{C} \backslash\left[\lim _{i \rightarrow \mathcal{U}} \mathbb{C} \backslash \sigma_{1 / n}\left(x_{i}\right)\right]\right]=\mathbb{C} \backslash \bigcup_{n \in \mathbb{N}} \lim _{i \rightarrow \mathcal{U}}\left[\mathbb{C} \backslash \sigma_{1 / n}\left(x_{i}\right)\right] \subseteq \sigma\left(\left(x_{i}\right) \mathcal{U}\right)
$$

Equivalently,

$$
\mathbb{C} \backslash \sigma\left(\left(x_{i}\right)_{\mathcal{U}}\right) \subseteq \bigcup_{n \in \mathbb{N}} \lim _{i \rightarrow \mathcal{U}}\left[\mathbb{C} \backslash \sigma_{1 / n}\left(x_{i}\right)\right]
$$


Proof. If $\lambda \notin \sigma\left(\left(x_{i}\right)_{\mathcal{U}}\right)$, then $\left(x_{i}-\lambda I\right)_{\mathcal{U}}$ is invertible, so there is an $M$ so that

$$
\left\{i: x_{i}-\lambda I \text { is inv. and }\left\|\left(x_{i}-\lambda I\right)\right\|<M\right\} \in \mathcal{U} \text {. }
$$

So $\left\{i \mid \lambda \notin \sigma_{1 / M}\left(x_{i}\right)\right\} \in \mathcal{U}$. So $\lambda \in \lim _{i \rightarrow \mathcal{U}} \mathbb{C} \backslash \sigma_{1 / M}\left(x_{i}\right) \subseteq \bigcup_{n \in \mathbb{N}} \lim _{i \rightarrow \mathcal{U}}\left[\mathbb{C} \backslash \sigma_{1 / n}\left(x_{i}\right)\right]$.

Thus we have

\section{Theorem 5.4.3.}

$$
\bigcap_{n \in \mathbb{N}}\left[\mathbb{C} \backslash\left[\lim _{i \rightarrow \mathcal{U}} \mathbb{C} \backslash \sigma_{1 / n}\left(x_{i}\right)\right]\right] \subseteq \sigma\left(\left(x_{i}\right)_{\mathcal{U}}\right) \subseteq \bigcap_{n \in \mathbb{N}} \lim _{\mathcal{U}} \sigma_{1 / n}\left(x_{i}\right)
$$

And we can take the union of these over all representative sequences $\left(y_{i}\right)_{\mathcal{U}}=\left(x_{i}\right)_{\mathcal{U}}$. The following is mentioned in Lemma 6 in [BN09]:

Proposition 5.4.4. Let $\mathcal{U}$ be an ultrafilter on $I$, and for $i \in I$ let $\mathfrak{A}_{i}$ be a unital $\mathrm{C}^{*}$-algebra. For $\left(x_{i}\right)_{\mathcal{U}} \in \prod_{\mathcal{U}} \mathfrak{A}_{i}, \lim _{\mathcal{U}} \sigma\left(x_{i}\right) \subseteq \sigma\left(\left(x_{i}\right)_{\mathcal{U}}\right)$.

Proof. Suppose $\lambda \in \lim _{\mathcal{U}} \sigma\left(x_{i}\right)$, then there are $\lambda_{i} \in \sigma\left(x_{i}\right)$ so that $\lim _{\mathcal{U}} \lambda_{i}=\lambda$. Then since

$$
\lim _{\mathcal{U}}\left\|\left(x_{i}-\lambda I\right)-\left(x_{i}-\lambda_{i} I\right)\right\|=\lim _{\mathcal{U}}\left|\lambda-\lambda_{i}\right|=0 .
$$

We have that

$$
\left(x_{i}-\lambda I\right)_{\mathcal{U}}=\left(x_{i}-\lambda_{i} I\right)_{\mathcal{U}}
$$

And the latter is non-invertible as $x_{i}-\lambda_{i} I$ is not invertible for all $i \in I$, as $\lambda_{i} \in \sigma\left(x_{i}\right)$. Thus $\lim _{\mathcal{U}} \sigma\left(x_{i}\right) \subseteq \sigma\left(\left(x_{i}\right)_{\mathcal{U}}\right)$ 
Proposition 5.4.5. If $x_{n} \in \mathcal{B}\left(\mathcal{H}_{n}\right)$ for all $n$, then for $\left(x_{n}\right)_{\mathcal{U}} \in \prod_{\mathcal{U}} \mathcal{B}\left(\mathcal{H}_{n}\right)$, viewed as an operator in $\mathcal{B}\left(\prod_{\mathcal{U}} \mathcal{H}_{n}\right)$, we have $r\left(\left(x_{n}\right)_{\mathcal{U}}\right) \geq \lim _{\mathcal{U}} r\left(x_{n}\right)$.

Proof. Since $\sigma\left(\left(x_{n}\right)_{\mathcal{U}}\right) \supseteq \lim _{\mathcal{U}} \sigma\left(x_{n}\right)$, we have

$$
r\left(\left(x_{n}\right)_{\mathcal{U}}\right)=\sup \left\{|\lambda|: \lambda \in \sigma\left(\left(x_{n}\right)_{\mathcal{U}}\right)\right\} \geq \sup \left\{|\lambda|: \lambda \in \lim _{\mathcal{U}} \sigma\left(x_{n}\right)\right\}=\lim _{\mathcal{U}} r\left(x_{n}\right)
$$

We will see shortly that this inequality can be strict.

Recall that the numerical range of an operator $x$ on a Hilbert space $\mathcal{H}$ is

$$
W(x)=\{\langle x \xi \mid \xi\rangle:\|\xi\|=1\}
$$

Proposition 5.4.6. Suppose $x_{n} \in \mathcal{B}\left(\mathcal{H}_{n}\right)$. For $\left(x_{n}\right)_{\mathcal{U}} \in \prod_{\mathcal{U}} \mathcal{B}\left(\mathcal{H}_{n}\right)$, viewed as an operator on $\mathcal{B}\left(\prod_{\mathcal{U}} \mathcal{H}_{n}\right), W\left(\left(x_{n}\right)_{\mathcal{U}}\right)=\lim _{\mathcal{U}} W\left(x_{n}\right)$.

Proof.

$$
\begin{aligned}
W\left(\left(x_{n}\right)_{\mathcal{U}}\right) & =\left\{\left\langle\left(x_{n}\right)_{\mathcal{U}}\left(\xi_{n}\right)_{\mathcal{U}} \mid\left(\xi_{n}\right)_{\mathcal{U}}\right\rangle:\left\|\left(\xi_{n}\right)\right\|=1\right\}=\left\{\lim _{\mathcal{U}}\left\langle x_{n} \xi_{n} \mid \xi_{n}\right\rangle: \xi_{n}=1 \text { for all } n\right\} \\
& =\left\{\lim _{\mathcal{U}} \lambda_{n}: \lambda_{n} \in W\left(x_{n}\right) \text { for all } n\right\}=\lim _{\mathcal{U}} W\left(x_{n}\right) .
\end{aligned}
$$

It is not the case that $(\mathcal{K}(\mathcal{H}))^{\mathcal{U}}$ is isomorphic to some $\mathcal{K}\left(\mathcal{H}_{0}\right)$, since self-adjoint compact operators have $c_{0}$ eigenvalues. 
Example 5.4.7. Let

$$
k_{n}=\left(\begin{array}{cccccc}
1 & 0 & \ldots & 0 & 0 & \ldots \\
0 & \frac{1}{2} & \ldots & 0 & 0 & \ldots \\
\vdots & \vdots & \ddots & \vdots & \vdots & \vdots \\
0 & 0 & \ldots & \frac{n-1}{n} & 0 & \ldots \\
0 & 0 & \ldots & 0 & 0 & \ldots \\
\vdots & \vdots & \vdots & \vdots & \vdots & \ddots
\end{array}\right) .
$$

Then consider $\left(k_{n}\right)_{\mathcal{U}}$, by the above,

$$
\sigma\left(\left(k_{n}\right) \mathcal{U}\right) \supseteq \lim _{\mathcal{U}} \sigma\left(k_{n}\right) \supseteq\left\{0, \frac{1}{2}, \frac{2}{3}, \frac{3}{4}, \frac{4}{5}, \ldots, 1\right\}
$$

Thus $\left(k_{n}\right)_{\mathcal{U}}$ is self-adjoint and its spectrum has 1 as a cluster point, so it is not compact.

The containment $\sigma\left(\left(x_{n}\right)_{\mathcal{U}}\right) \supseteq \lim _{\mathcal{U}} \sigma\left(x_{n}\right)$ can be proper:

Example 5.4.8. For $n \in \mathbb{N}$ consider

$$
x_{n}=\left(\begin{array}{ccccc}
0 & 0 & 0 & \ldots & 0 \\
1 & 0 & 0 & \ldots & 0 \\
0 & 1 & 0 & \ldots & 0 \\
\vdots & \vdots & \ddots & \ddots & \vdots \\
0 & 0 & \ldots & 1 & 0
\end{array}\right) \in M_{n} .
$$

Note that for $n \geq 2, x_{n}^{n}=0$, but for $k<n,\left\|x_{n}^{k}\right\|=1$, since it sends the $k^{\text {th }}$ basis vector $e_{k}$ to $e_{1}$. Since

$$
r\left(x_{n}\right)=\lim _{k \rightarrow \infty}\left\|x_{n}^{k}\right\|^{1 / k}=0,
$$


the spectrum of each $x_{n}$ is $\{0\}$. However, considering $\left(x_{n}\right)_{\mathcal{U}}$ in $\prod_{\mathcal{U}} M_{n}$, we have that

$$
r\left(\left(x_{n}\right)_{\mathcal{U}}\right)=\lim _{k \rightarrow \infty}\left\|\left(x_{n}\right)_{\mathcal{U}}^{k}\right\|=\lim _{k \rightarrow \infty} \lim _{\mathcal{U}}\left\|x_{n}^{k}\right\|=1
$$

In fact, if $\alpha \in \mathbb{C}$ with $0<|\alpha| \leq 1$, then

$$
x_{n}+\alpha I_{n}=\left(\begin{array}{ccccc}
\alpha & 0 & 0 & \ldots & 0 \\
1 & \alpha & 0 & \ldots & 0 \\
0 & 1 & \alpha & \ldots & 0 \\
\vdots & \vdots & \ddots & \ddots & \vdots \\
0 & 0 & \ldots & 1 & \alpha
\end{array}\right) .
$$

One can calculate that

$$
\left(x_{n}+\alpha I_{n}\right)^{-1}=\frac{1}{\alpha}\left(\begin{array}{ccccc}
1 & 0 & 0 & \ldots & 0 \\
-1 / \alpha & 1 & 0 & \ldots & 0 \\
1 / \alpha^{2} & -1 / \alpha & 1 & \ldots & 0 \\
\vdots & \vdots & \ddots & \ddots & \vdots \\
(-1 / \alpha)^{n} & (-1 / \alpha)^{n-1} & \ldots & -1 / \alpha & 1
\end{array}\right),
$$

which has norm at least $\sqrt{n}$ by looking at the first column. In particular, $\left(x_{n}+\alpha I_{n}\right)_{\mathcal{U}}$ is not invertible, so $-\alpha \in \sigma\left(\left(x_{n}\right)_{\mathcal{U}}\right)$.

It is not the case that if $\left(x_{n}\right)_{\mathcal{U}}=\left(y_{n}\right)_{\mathcal{U}}$ then $\lim _{\mathcal{U}} \sigma\left(x_{n}\right)=\lim _{\mathcal{U}} \sigma\left(y_{n}\right)$ :

Example 5.4.9 (102 in [Hal82]). Consider the weighted shifts $s_{k}$ for $k \geq 1$ on $\ell^{2}(\mathbb{Z})$ with basis $\left\{e_{n}\right\}_{n \in \mathbb{Z}}$ defined by

$$
s_{k} e_{j}=e_{j+1} \quad \text { if } j \neq 0, \quad \text { and } \quad s_{k} e_{0}=\frac{1}{k} e_{1}
$$


Then $\sigma\left(s_{k}\right)=\{\lambda:|\lambda|=1\}=\mathbb{T}$, and $\lim _{k \rightarrow \infty}\left\|s_{k}-s_{\infty}\right\|=0$ where $s_{\infty}$ is the weighted shift defined by

$$
s_{\infty} e_{j}=e_{j+1} \quad \text { if } j \neq 0, \quad \text { and } \quad s_{\infty} e_{0}=0 .
$$

Then $\sigma\left(s_{\infty}\right)=\{\lambda:|\lambda| \leq 1\}=\mathbb{D}$. In particular $\left(s_{k}\right)_{\mathcal{U}}=\left(s_{\infty}\right)_{\mathcal{U}}$, but

$$
\lim _{\mathcal{U}} \sigma\left(s_{k}\right)=\mathbb{T} \neq \mathbb{D}=\lim _{\mathcal{U}} \sigma\left(s_{\infty}\right)
$$

Proposition 5.4.10. Let $\mathcal{U}$ be a free ultrafilter on $\mathbb{N}$, and for all $n \in \mathbb{N}$ let $\mathfrak{A}_{n} \subseteq$ $\mathcal{B}\left(\mathcal{H}_{n}\right)$ be a concrete $\mathrm{C}^{*}$-algebra. Let $\left(x_{n}\right)_{\mathcal{U}} \in \prod_{\mathcal{U}} \mathfrak{A}_{n}$ represented in $\mathcal{B}\left(\prod_{\mathcal{U}} \mathcal{H}_{n}\right)$. If $\lambda \in \lim _{\mathcal{U}} \sigma\left(x_{n}\right)$ then $\lambda$ is either an eigenvalue of $\left(x_{n}\right)_{\mathcal{U}}$ or $\bar{\lambda}$ is an eigenvalue of $\left(x_{n}^{*}\right)_{\mathcal{U}}$.

Proof. Recall that $\lambda \in \sigma(x)$ is either an eigenvalue of $x$, an approximate eigenvalue for $x$, or $\bar{\lambda}$ is an approximate eigenvalue of $x^{*}$. Suppose $\lambda \in \lim _{\mathcal{U}} \sigma\left(x_{n}\right)$, then take $\lambda_{n} \in \sigma\left(x_{n}\right)$ so $\lambda=\lim _{\mathcal{U}} \lambda_{n}$. If $\lambda_{n}$ is an eigenvector of $x_{n}$, pick a unit vector $\xi_{n}$ so $x_{n} \xi_{n}=\lambda_{n} \xi_{n}$. If $\lambda_{n}$ is an approximate eigenvector of $x_{n}$, pick a unit vector $\xi_{n}$ so $\left\|x_{n} \xi_{n}-\lambda \xi_{n}\right\|<1 / n$. If $\bar{\lambda}_{n}$ is an approximate eigenvector of $x_{n}^{*}$, pick a unit vector $\xi_{n}$ so $\left\|x_{n}^{*} \xi_{n}-\bar{\lambda}_{n} \xi_{n}\right\|<1 / n$. Consider $\left(\xi_{n}\right) \mathcal{U}$.

If $\left\{n \mid \lambda_{n}\right.$ is an eigenvalue or an approximate eigenvalue of $\left.x_{n}\right\} \in \mathcal{U}$, then

$$
\left\|\left(x_{n} \xi_{n}\right)_{\mathcal{U}}-\lambda\left(\xi_{n}\right)_{\mathcal{U}}\right\|=\lim _{\mathcal{U}}\left\|x_{n} \xi_{n}-\lambda \xi_{n}\right\|=\lim _{\mathcal{U}}\left\|x_{n} \xi_{n}-\lambda_{n} \xi_{n}\right\|=0
$$

Thus $\left(x_{n} \xi_{n}\right)_{\mathcal{U}}=\lambda\left(\xi_{n}\right)_{\mathcal{U}}$, so $\lambda$ is an eigenvector of $\left(x_{n}\right)_{\mathcal{U}}$.

Otherwise, $\left\{n \mid \bar{\lambda}_{n}\right.$ is an approximate eigenvalue of $\left.x_{n}^{*}\right\} \in \mathcal{U}$, and similarly $\left(x_{n}^{*} \xi_{n}\right)_{\mathcal{U}}=$ $\bar{\lambda}\left(\xi_{n}\right)_{\mathcal{U}}$, so $\bar{\lambda}$ is an eigenvalue of $\left(x_{n}^{*}\right)_{\mathcal{U}}$. 
Comment: this, and the other proofs for ultrafilters on the natural numbers can be adapted for countably incomplete ultrafilters, by replacing the set $\{n \mid n \geq N\}$ with the set $A_{N}$ from a countable sequence $A_{n} \in \mathcal{U}$ with $\bigcap_{n \in \mathbb{N}} A_{n}=\emptyset$.

We have the following converse, which is similar to Theorem 3.1 in [Moo76].

Proposition 5.4.11. Let $\mathcal{U}$ be an free ultrafilter on $\mathbb{N}$. If $\lambda$ is an approximate eigenvalue of $\left(x_{n}\right)_{\mathcal{U}} \in \prod_{\mathcal{U}} \mathcal{B}\left(\mathcal{H}_{n}\right)$, viewed as an operator on $\mathcal{B}\left(\prod_{\mathcal{U}} \mathcal{H}_{n}\right)$, then $\lambda$ is an is an eigenvalue of $\left(x_{n}\right)_{\mathcal{U}}$. Furthermore there is a representative sequence $\left(y_{n}\right)_{\mathcal{U}}=\left(x_{n}\right)_{\mathcal{U}}$ so that $\lambda \in \sigma\left(y_{n}\right)$ for all $n \in \mathbb{N}$.

Proof. Suppose $\lambda$ is an approximate eigenvalue of $\left(x_{n}\right)_{\mathcal{U}}$. Then for all $k \in \mathbb{N}$, there is a unit vector $\left(\xi_{n}^{(k)}\right)_{n \rightarrow \mathcal{U}}$ so $\left\|\left(x_{n} \xi_{n}^{(k)}\right)_{n \rightarrow \mathcal{U}}-\lambda\left(\xi_{n}^{(k)}\right)_{n \rightarrow \mathcal{U}}\right\|<1 / k$ (and without loss of generality we can assume $\left\|\xi_{n}^{(k)}\right\| \leq 1$ for all $\left.n, k\right)$. Let

$$
U_{k}=\left\{n:\left\|x_{n} \xi_{n}^{(k)}-\lambda \xi_{n}^{(k)}\right\|<\frac{1}{k}\right\} \in \mathcal{U}
$$

Let $V_{1}=U_{1}$, and $V_{k}=\bigcap_{j=1}^{k} U_{k} \in \mathcal{U}$. For $n \notin V_{1}$, let $\eta_{n}=\xi_{n}^{(1)}$. For $n \in V_{k} \backslash V_{k+1}$, let $\eta_{n}=\xi_{n}^{(k)}$. Finally, if $n \in \bigcap_{k} V_{k}$, then there is a $k$ so

$$
\left\|x_{n} \xi_{n}^{(k)}-\lambda \xi_{n}^{(k)}\right\|<\frac{1}{n}
$$

We let $\eta_{n}=\xi_{n}^{(k)}$. Consider $\left(\eta_{n}\right)_{\mathcal{U}}$, then since for $n \in\{n \mid n \geq N\} \cap V_{N} \in \mathcal{U}, \| x_{n} \eta_{n}-$ $\lambda \eta_{n} \|<2 / N$, we have that

$$
\left\|\left(x_{n} \eta_{n}\right)_{\mathcal{U}}-\lambda\left(\eta_{n}\right)_{\mathcal{U}}\right\|=\lim _{\mathcal{U}}\left\|x_{n} \eta_{n}-\lambda \eta_{n}\right\|=0
$$

Thus $\lambda$ is an eigenvector of $\left(x_{n}\right)_{\mathcal{U}}$. 
Conversely, if $\lambda$ is an eigenvalue of $\left(x_{n}\right)_{\mathcal{U}}$, then there is a vector $\left(\xi_{n}\right)_{\mathcal{U}}$ so that $\left(x_{n} \xi_{n}\right)=\left(\lambda \xi_{n}\right)_{\mathcal{U}}$, so $\lim _{\mathcal{U}}\left\|x_{n} \xi_{n}-\lambda \xi_{n}\right\|=0$. Let

$$
y_{n}=x_{n}\left(I-p_{\xi_{n}}\right)+\lambda p_{\xi_{n}},
$$

where $p_{\xi_{n}}$ is the rank 1 projection onto the span of $\xi_{n}$. Then

$$
\left\|\left(x_{n}\right)_{\mathcal{U}}-\left(y_{n}\right)_{\mathcal{U}}\right\|=\lim _{\mathcal{U}}\left\|x_{n} p_{\xi_{n}}-\lambda p_{\xi_{n}}\right\|=0
$$

So $\left(y_{n}\right)_{\mathcal{U}}=\left(x_{n}\right)_{\mathcal{U}}$ and $y_{n} \xi_{n}=\lambda \xi_{n}$ for all $n$. Thus $\lambda \in \sigma\left(y_{n}\right)$ for all $n$ and $\lambda \in \lim _{\mathcal{U}} \sigma\left(y_{n}\right)$ for all $n$.

Comment: It suffices to consider subalgebras of $\mathcal{B}\left(\mathcal{H}_{n}\right)$ which contain the compact operators.

Corollary 5.4.12. Let $\mathcal{U}$ be a free ultrafilter on $\mathbb{N}$. Then for $\left(x_{n}\right)_{\mathcal{U}} \in \prod_{\mathcal{U}} \mathcal{B}\left(\mathcal{H}_{n}\right)$,

$$
\sigma\left(\left(x_{n}\right)_{\mathcal{U}}\right)=\bigcup_{\left(y_{n}\right)_{\mathcal{U}}=\left(x_{n}\right)_{\mathcal{U}}} \lim _{\mathcal{U}} \sigma\left(y_{n}\right)
$$

Proof. If $\lambda \in \sigma\left(\left(x_{n}\right)_{\mathcal{U}}\right)$ then either $\lambda$ is an eigenvalue of $\left(x_{n}\right)_{\mathcal{U}}$ or $\lambda$ is an eigenvalue of $\left(x_{n}^{*}\right)_{\mathcal{U}}$. In the first case there is a $\left(y_{n}\right)_{\mathcal{U}}=\left(x_{n}\right)_{\mathcal{U}}$ so $\lambda \in \sigma\left(y_{n}\right)$ for all $n$. Otherwise there is a $\left(z_{n}^{*}\right)_{\mathcal{U}}=\left(x_{n}^{*}\right)_{\mathcal{U}}$, so that $\bar{\lambda} \in \sigma\left(z_{n}^{*}\right)$ for all $n$. Thus $\lambda \in \sigma\left(z_{n}\right)$ for all $n$ and $\left(z_{n}\right)_{\mathcal{U}}=\left(x_{n}\right)_{\mathcal{U}}$

This raises the question:

Question 5.4.13. Given $\left(x_{n}\right)_{\mathcal{U}} \in \prod_{\mathcal{U}} \mathcal{B}\left(\mathcal{H}_{n}\right)$, viewed as an operator in $\mathcal{B}\left(\prod_{\mathcal{U}} \mathcal{H}_{n}\right)$ can we find $\left(y_{n}\right)_{\mathcal{U}}=\left(x_{n}\right)_{\mathcal{U}}$ such that $\sigma\left(\left(x_{n}\right)_{\mathcal{U}}\right)=\lim _{\mathcal{U}} \sigma\left(y_{n}\right) ?$ 
Proposition 5.4.14. Let $\mathcal{U}$ be a free ultrafilter on $\mathbb{N}$. Suppose $\left(x_{n}\right)_{\mathcal{U}} \in \prod_{\mathcal{U}} \mathcal{B}\left(\mathcal{H}_{n}\right)$ is normal and each $\mathcal{H}_{n}$ is infinite-dimensional. Then there is a $\left(y_{n}\right)_{\mathcal{U}}=\left(x_{n}\right)_{\mathcal{U}}$ so $\lim _{\mathcal{U}} \sigma\left(y_{n}\right)=\sigma\left(\left(x_{n}\right)_{\mathcal{U}}\right)$

Proof. As usual we view $\left(x_{n}\right)_{\mathcal{U}}$ as an operator in $\mathcal{B}\left(\prod_{\mathcal{U}} \mathcal{H}_{n}\right)$. Note that if $\lambda \in$ $\sigma\left(\left(x_{n}\right) \mathcal{U}\right)$, it is either an approximate eigenvalue of $\left(x_{n}\right)_{\mathcal{U}}$ or $\bar{\lambda}$ is an eigenvector for $\left(x_{n}\right)_{\mathcal{U}}^{*}$. Since $\left(x_{n}\right)_{\mathcal{U}}$ is normal, these operators are metrically equivalent. Thus we can assume $\lambda$ is an approximate eigenvalue for $\left(x_{n}\right)_{\mathcal{U}}$, so it is actually an eigenvalue. Thus we can take a countable collection $\left\{\lambda_{j}\right\}$ of eigenvalues which is dense in $\sigma\left(\left(x_{n}\right)_{\mathcal{U}}\right)$. Let $\left.{ }^{\left(\xi_{n}^{(j)}\right.}\right)_{\mathcal{U}} \in \prod_{\mathcal{U}} \mathcal{H}_{n}$ be an eigenvector for $\left(x_{n}\right)_{\mathcal{U}}$ corresponding to $\lambda_{j}$. By the fact $\left(x_{n}\right)_{\mathcal{U}}$ is normal these eigenvectors are orthogonal. By 3.1.2 we can take representations so for $i \neq j, \xi_{n}^{i}$ and $\xi_{n}^{j}$ are orthogonal, for all $n$. Now we let

$$
A_{j, k}=\left\{n:\left\|x_{n} \xi_{n}^{(j)}-\lambda_{j} \xi_{n}^{(j)}\right\|<2^{-k}\right\} \in \mathcal{U}
$$

Then let $B_{1}=A_{1,1}$,

$$
B_{k}=\bigcap_{1 \leq j \leq k} A_{j, k}=\left\{n:\left\|x_{n} \xi_{n}^{(j)}-\lambda_{j} \xi_{n}^{(j)}\right\|<2^{-k} \text { for } 1 \leq j \leq k\right\} \in \mathcal{U}
$$

For $n \notin B_{1}$ let $y_{n}=x_{n}$. For $n \in B_{k} \backslash B_{k+1}$, let

$$
y_{n}=x_{n}-x_{n} \sum_{1 \leq j \leq k} p_{\xi_{n}^{(j)}}+\sum_{1 \leq j \leq k} \lambda_{j} p_{\xi_{n}^{(j)}}
$$

Since the $\xi_{n}^{j}$ are orthogonal, $\lambda_{j} \in \sigma\left(y_{n}\right)$ for $1 \leq j \leq n$. Note for $n \in B_{k} \backslash B_{k+1}$,

$$
\left\|x_{n}-y_{n}\right\|=\left\|\sum_{1 \leq j \leq k} x_{n} p_{\xi_{n}^{(j)}}-\lambda_{j} p_{\xi_{n}^{(j)}}\right\| \leq \sum_{1 \leq j \leq k}\left\|x_{n} \xi_{n}^{(j)}-\lambda_{j} \xi_{n}^{(j)}\right\| \leq \sum_{1 \leq k \leq j} \frac{1}{2^{k}}=\frac{k}{2^{k}}
$$


For $n \in \bigcap_{k} B_{k}$, we must have $x_{n} \xi_{n}^{(j)}=\lambda_{j} \xi_{n}^{(j)}$ for all $j$, and we let $y_{n}=x_{n}$. Then $\left(y_{n}\right)_{\mathcal{U}}=\left(x_{n}\right)_{\mathcal{U}}$ and

$$
\left\{\lambda_{k}\right\}_{k \in \mathbb{N}} \subseteq \lim _{\mathcal{U}} \sigma\left(y_{n}\right)=\subseteq \sigma\left(\left(y_{n}\right)_{\mathcal{U}}\right)=\sigma\left(\left(x_{n}\right)_{\mathcal{U}}\right)
$$

Since spectra are compact, the closure of $\left\{\lambda_{k}\right\}_{k \in \mathbb{N}}=\sigma\left(\left(x_{n}\right)_{\mathcal{U}}\right)$ is contained in $\lim _{\mathcal{U}} \sigma\left(y_{n}\right)$, so we can conclude that

$$
\lim _{\mathcal{U}} \sigma\left(y_{n}\right)=\sigma\left(\left(x_{n}\right)_{\mathcal{U}}\right)
$$

There is one case when we can say $\sigma\left(\left(x_{n}\right)_{\mathcal{U}}\right)=\lim _{\mathcal{U}} \sigma\left(x_{n}\right)$. We will need the following:

Proposition 5.4.15. If $x$ is a normal element in a unital $\mathrm{C}^{*}$-algebra, and $\operatorname{dist}(\lambda, \sigma(x))=$ $\varepsilon>0$ then $\left\|(x-\lambda I)^{-1}\right\| \leq 1 / \varepsilon$.

Proof. This is a simple application of the spectral theorem. Since $\lambda \notin \sigma(x), x-\lambda I$ is invertible. Letting $f(t)=1 /(t-\lambda)$, note that $|f(t)| \leq 1 /|t-\lambda| \leq 1 / \varepsilon$ for $t \in \sigma(x)$, then we have

$$
\left\|(x-\lambda I)^{-1}\right\|=\|f\|_{\infty, \sigma(x)} \leq \frac{1}{\varepsilon}
$$

In fact,

Proposition 5.4.16 (4.7 in [Con91]). If $x$ is a hyponormal element (that is, $x^{*} x-$ $\left.x x^{*} \geq 0\right)$ and $\lambda \notin \sigma(A)$, then $\left\|(x-\lambda I)^{-1}\right\|=(\operatorname{dist}(\lambda, \sigma(A)))^{-1}$.

In particular, for normal or hyponormal $x, \sigma(x) \subseteq \sigma_{\varepsilon}(x) \subseteq \sigma(x)_{\varepsilon}$. The following is an extension of Lemma 6 in [BN09]: 
Theorem 5.4.17. Let $\mathcal{U}$ be an ultrafilter on $I$ and for all $i \in I$ let $\mathfrak{A}_{i}$ be a unital $\mathrm{C}^{*}$-algebra. If $x_{i}$ are uniformly bounded normal or hyponormal elements in $\mathfrak{A}_{i}$, then $\lim _{\mathcal{U}} \sigma\left(x_{i}\right)=\sigma\left(\left(x_{i}\right)_{\mathcal{U}}\right)$

Proof. Suppose $\lambda \notin \lim _{\mathcal{U}} \sigma\left(x_{i}\right)$. Since $\lim _{\mathcal{U}} \sigma\left(x_{i}\right)$ is compact, $\operatorname{dist}\left(\lim _{\mathcal{U}} \sigma\left(x_{i}\right), \lambda\right)=$ $\varepsilon_{0}>0$. Thus every sequence $\lambda_{i} \in \sigma\left(x_{i}\right), \lim _{\mathcal{U}}\left|\lambda_{i}-\lambda\right| \geq \varepsilon_{0}$. Thus

$$
U=\left\{i:\left\|\lambda-\sigma\left(x_{i}\right)\right\|>\varepsilon_{0} / 2\right\} \in \mathcal{U}
$$

Then for $i \in U$, we have that $\operatorname{dist}\left(\sigma\left(x_{i}\right), \lambda\right)>\varepsilon_{0} / 2$, so $x_{i}-\lambda I$ is invertible, and $\left\|\left(x_{i}-\lambda I\right)^{-1}\right\| \leq 2 / \varepsilon_{0}$ are uniformly bounded, so $\left(\left(x_{i}-\lambda I\right)^{-1}\right)_{\mathcal{U}}$ is defined. Thus $\left(x_{i}-\lambda I\right)_{\mathcal{U}}$ is invertible, so $\lambda \notin \sigma\left(\left(x_{i}\right)_{\mathcal{U}}\right)$.

Comment: it does not suffice that $\left(x_{i}\right)_{\mathcal{U}}$ is normal, we need a representation where each $x_{i}$ is normal. This is closely related to Lin's Theorem.

This does not mean that every representative sequence of a normal element $\left(x_{i}\right)_{\mathcal{U}}$ has $\lim _{\mathcal{U}} \sigma\left(x_{i}\right)=\sigma\left(\left(x_{i}\right)_{\mathcal{U}}\right)$ : 
Example 5.4.18. Let

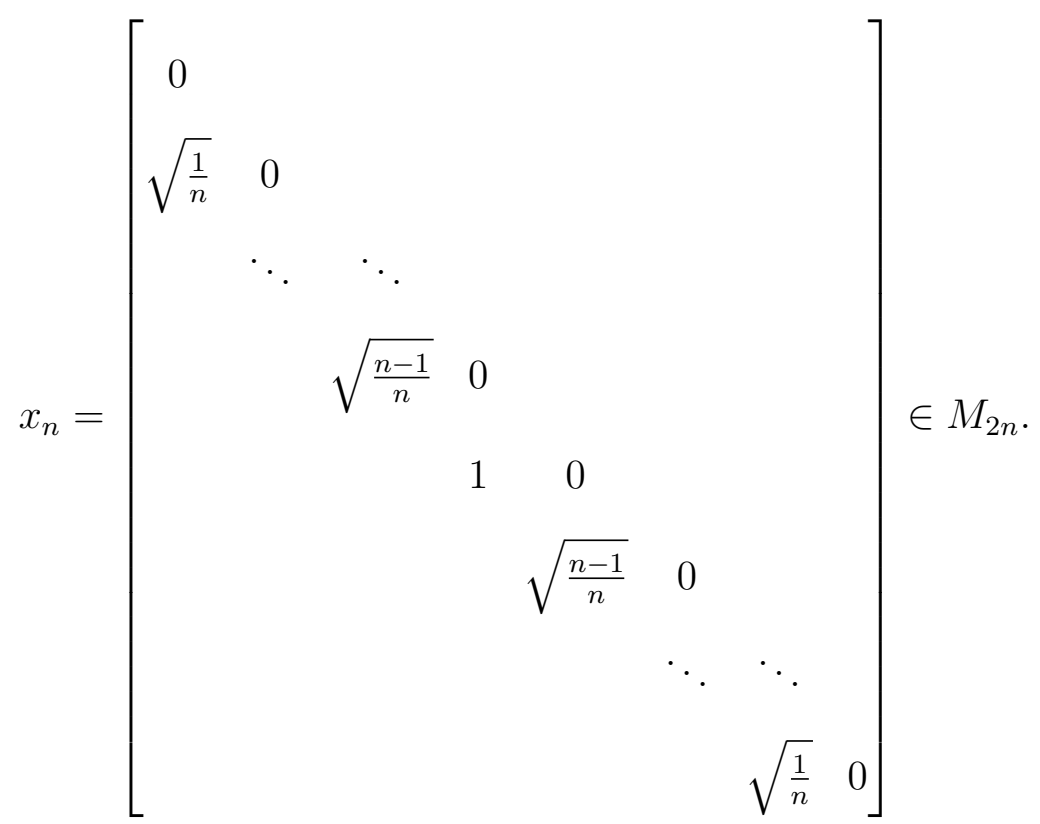

Note that $x_{n}$ is nilpotent so $\sigma\left(x_{n}\right)=\{0\}$. Also

$$
x_{n}^{*} x_{n}=\operatorname{diag}\left(\frac{1}{n}, \ldots, \frac{n-1}{n}, 1, \frac{n-1}{n}, \ldots, \frac{1}{n}, 0\right),
$$

so $\left\|x_{n}\right\|=1$. Similarly,

$$
x_{n} x_{n}^{*}=\operatorname{diag}\left(0, \frac{1}{n} \ldots, 1, \ldots \frac{1}{n}\right) .
$$

Thus we have that

$$
\begin{aligned}
x_{n}^{*} x_{n}-x_{n} x_{n}^{*} & =\operatorname{diag}\left(\frac{1}{n}-0, \frac{2}{n}-\frac{1}{n}, \ldots, 1-\frac{n-1}{n}, \frac{n-1}{n}-1, \ldots,-\frac{1}{n}\right) \\
& =\operatorname{diag}\left(\frac{1}{n}, \frac{1}{n}, \ldots, \frac{1}{n},-\frac{1}{n}, \ldots,-\frac{1}{n}\right) .
\end{aligned}
$$

In particular, $\left\|\left[x_{n}, x_{n}^{*}\right]\right\|=1 / n$. Thus $\left(x_{n}\right)_{\mathcal{U}} \in \prod_{\mathcal{U}} M_{2 n}$ is normal and $r\left(\left(x_{n}\right)_{\mathcal{U}}\right)=$ 
$\left\|\left(x_{n}\right)_{\mathcal{U}}\right\|=1$. In fact, for $|\alpha|<1$,

$$
\left(x_{n}+\alpha I\right)\left(\begin{array}{c}
\frac{1}{\alpha} \\
-\frac{1}{\alpha^{2}} \sqrt{\frac{1}{n}} \\
\vdots \\
(-1)^{k} \frac{1}{\alpha^{k}} \sqrt{\frac{k !}{n^{k}}} \\
\vdots \\
(-1)^{n} \frac{1}{\alpha^{2 n}} \sqrt{\frac{n ! 2}{n^{2 n}}}
\end{array}\right)=\left(\begin{array}{c}
1 \\
0 \\
\vdots \\
0
\end{array}\right) .
$$

In particular, $\left\|\left(x_{n}+\alpha I\right)^{-1}\right\| \geq\left\|\frac{1}{\alpha^{n}} \frac{1}{\sqrt{n}}\right\| \rightarrow \infty$. So $-\alpha \in \sigma\left(\left(x_{n}\right)_{\mathcal{U}}\right)$ for all $|\alpha| \leq 1$.

Question 5.4.19. Given a normal element $\left(x_{n}\right)_{\mathcal{U}} \in \prod_{\mathcal{U}} \mathfrak{A}_{n}$, can we tell when there are normal $t_{n}$ so $\left(x_{n}\right) \mathcal{U}=\left(t_{n}\right) \mathcal{U}$ ?

One might call such elements in ultraproducts ultra-normal. The next Chapter focuses on this question of when normal elements have normal representative sequences. 


\section{Chapter 6}

\section{Lin's Theorem and Ultraproducts}

We study when normal elements in ultraproducts have normal representative sequences. If $N(\mathfrak{A})$ denotes the normal elements of a $\mathrm{C}^{*}$-algebra $\mathfrak{A}$, it is clear that $N\left(\prod_{\mathcal{U}} A_{i}\right) \supseteq \prod_{\mathcal{U}} N\left(\mathfrak{A}_{i}\right)$. We will see this containment can be proper and see some examples when equality holds.

\subsection{Counterexamples}

It is not the case that if $\left(x_{n}\right) \mathcal{U}$ is normal that we can necessarily find a representative sequence of normal elements $y_{n}$ so $\left(y_{n}\right)_{\mathcal{U}}=\left(x_{n}\right)_{\mathcal{U}}$ :

Example 6.1.1 ([Hal77]). Consider the tapered unilateral shifts $s_{n}(n \geq 1)$ on $\ell_{2}(\mathbb{N})$ with basis $\left\{\xi_{k}: k \geq 1\right\}$ given by

$$
s_{n} \xi_{k}=\sqrt{\frac{\min (k, n)}{n}} \xi_{k+1} .
$$


Then

$$
s_{n}^{*} \xi_{k}=\sqrt{\frac{\min (k-1, n)}{n}} \xi_{k-1} .
$$

Now we can calculate

$$
\begin{aligned}
{\left[s_{n}^{*}, s_{n}\right] \xi_{k} } & =s_{n}^{*} s_{n} \xi_{k}-s_{n} s_{n}^{*} \xi_{k}=s_{n}^{*} \sqrt{\frac{\min (k, n)}{n}} \xi_{k+1}-s_{n} \sqrt{\frac{\min (k-1, n)}{n}} \xi_{k-1} \\
& =\frac{\min (k, n)}{n} \xi_{k}-\frac{\min (k-1, n)}{n} \xi_{k}= \begin{cases}\frac{1}{n} \xi_{k}, & 1 \leq k \leq n \\
0, & \text { otherwise }\end{cases}
\end{aligned}
$$

Thus $\left[s_{n}^{*}, s_{n}\right]=\frac{1}{n} p_{n}$ where $p_{n}$ is the projection onto $\operatorname{span}\left(\xi_{1}, \ldots, \xi_{n}\right)$ so $\left\|\left[s_{n}^{*}, s_{n}\right]\right\|=$ $1 / n \rightarrow 0$, but $s_{n}$ is a compact perturbation of the unilateral shift $s$, so this operator is Fredholm of index -1 . It can be shown that if $t$ is an operator with $\left\|s_{n}-t\right\|<1$ then $t$ has Fredholm index -1 . A normal Fredholm operator $x$ has index $\operatorname{dim}(\operatorname{ker}(x))-$ $\operatorname{dim}\left(\operatorname{ker}\left(x^{*}\right)\right)=0$, because $\operatorname{ker}(x)=\operatorname{ker}\left(x^{*}\right)$. Thus for all $n, s_{n}$ is at least distance 1 from any normal operator. Thus if $\mathcal{U}$ is a free ultrafilter on $\mathbb{N}$, then $\left(s_{n}\right)_{\mathcal{U}}$ is a normal element in $\mathcal{B}\left(\ell^{2}(\mathbb{N})\right)^{\mathcal{U}}$ with no representative sequence of normal elements.

It is not the case that if $\left(x_{n}\right)_{\mathcal{U}}$ and $\left(y_{n}\right)_{\mathcal{U}}$ commute that we can find representative sequences which commute coordinate-wise:

Theorem 6.1.2 ([Cho88]). For all $n$, there are $a_{n}, b_{n} \in M_{n}$ with $\left\|a_{n}\right\|=1-1 / n$, $\left\|b_{n}\right\|=1,\left\|\left[a_{n}, b_{n}\right]\right\| \leq 2 / n$, and for all commuting $r_{n}, s_{n} \in M_{n},\left\|a_{n}-r_{n}\right\|+\| b_{n}-$ $s_{n} \| \geq 1-1 / n$

In particular, if $\mathcal{U}$ is a free ultrafilter on $\mathbb{N}$ then $\left(a_{n}\right)_{\mathcal{U}}$ and $\left(b_{n}\right)_{\mathcal{U}}$ are commuting elements of $\prod_{\mathcal{U}} M_{n}$ which have no commuting representative sequences. 
Even commuting self-adjoint elements can fail to have commuting representative sequences (consider $\left(\Re\left(s_{n}\right)\right)_{\mathcal{U}}$ and $\left(\Im\left(s_{n}\right)\right)_{\mathcal{U}}$ in Example 6.1.1). Likewise, commuting unitaries can fail to have commuting representative sequences:

Example 6.1.3 ([Voi83]). Let $u_{n}, v_{n} \in M_{n}$ with basis $\xi_{1}, \ldots \xi_{n}$ be the unitaries defined by

$$
u_{n} \xi_{k}=\xi_{k+1}(\bmod n) \quad v_{n} \xi_{k}=e^{2 k \pi i / n} \xi_{k}
$$

One can calculate that $u_{n} v_{n}=e^{-2 \pi i / n} v_{n} u_{n}$. Thus $\lim _{n \rightarrow \infty}\left\|\left[u_{n}, v_{n}\right]\right\|=0$. It turns out that there are no commuting unitaries $u_{n}^{\prime}, v_{n}^{\prime} \in M_{n}$ with $\lim _{n \rightarrow \infty}\left\|u_{n}-u_{n}^{\prime}\right\|+\| v_{n}-$ $v_{n}^{\prime} \|=0$. In fact, Exel and Loring showed in [EL89] for any commuting unitaries $u_{n}^{\prime}$ and $v_{n}^{\prime}$

$$
\left\|u_{n}-u_{n}^{\prime}\right\|+\left\|v_{n}-v_{n}^{\prime}\right\| \geq \sqrt{2-\left|1-e^{2 \pi i / n}\right|}-1 \rightarrow \sqrt{2}-1 .
$$

Thus if $\mathcal{U}$ is a free ultrafilter on $\mathbb{N}$ then $\left(u_{n}\right)_{\mathcal{U}}$ and $\left(v_{n}\right)_{\mathcal{U}}$ are commuting unitaries with no commuting representative sequences.

\subsection{Lin's Property and Lin's Theorem}

Definition 6.2.1. We say that a $\mathrm{C}^{*}$-algebra $\mathfrak{A}$ has Lin's property if for all $\varepsilon>0$, there is a $\delta>0$ such that for any $x \in \mathfrak{A}$ with $\|x\| \leq 1$ and $\left\|\left[x^{*}, x\right]\right\|<\delta$, then there is a normal $y \in \mathfrak{A}$ (so $\left\|\left[y^{*}, y\right]\right\|=0$ ) with $\|y-x\|<\varepsilon$. Intuitively, Lin's property says "almost normal elements are near to normal elements".

Equivalently, (by considering real and complex parts) $\mathfrak{A}$ has Lin's property if for 
all $\varepsilon>0$, there is a $\delta>0$ such that for any self-adjoint $x, y \in \mathfrak{A}$ with $\|x\|,\|y\| \leq 1$ and $\|[x, y]\|<\delta$, then there are self-adjoint elements $x^{\prime}, y^{\prime} \in \mathfrak{A}$ with $\left\|x-x^{\prime}\right\|+\left\|y-y^{\prime}\right\|<\varepsilon$. Intuitively, this says "almost commuting self-adjoint elements are near to commuting self-adjoint elements".

Rosenthal asked whether matrix algebras $M_{n}(\mathbb{C})$ have Lin's property [Ros69]. This is the case:

Theorem 6.2.2 ([LT70]). Any finite-dimensional $\mathrm{C}^{*}$-algebra has Lin's property.

Proof. For sake of contradiction, suppose $M_{n}$ does not have Lin's property. Then there is a $\varepsilon>0$ such that for all $k$, there is some $x_{k} \in M_{n}$ with $\left\|x_{k}\right\| \leq 1$ and $\left\|\left[x_{k}, x_{k}^{*}\right]\right\|<1 / k$ but for every normal $y \in M_{n},\left\|x_{k}-y\right\| \geq \varepsilon$. Since $M_{n}$ is finitedimensional, the unit ball of $M_{n}$ is (sequentially) compact. Thus by considering a subsequence of the $x_{k}$ we can assume the $x_{k}$ converge to some $x \in M_{n}$. Then $\|x\| \leq 1$ and for all $k \in \mathbb{N}$,

$$
\left\|\left[x, x^{*}\right]\right\| \leq 2\left(\|x\|+\left\|x_{k}\right\|\right)\left\|x_{k}-x\right\|+\left\|\left[x_{k}, x_{k}^{*}\right]\right\| .
$$

Since the right-hand side tends to zero as $k$ grows, $x$ is normal. This is a contradiction since the $x_{k}$ get arbitrarily close to $x$.

The stronger question of whether the $\delta$ could be taken independent of the dimension $n$ was popularized by Halmos [Hal77]. Lin showed this to be the case [Lin97], and a short, elementary proof of the result was given by Friis and Rørdam [FR96]. 
Theorem 6.2.3 (Lin's Theorem). $\prod_{\mathbb{N}} M_{n}$ has Lin's property. More precisely, for all $\varepsilon>0$ there is a $\delta>0$ (independent of the dimension $n$ ) so that for any $n \in \mathbb{N}$, for all $x \in M_{n}$ with $\|x\| \leq 1$ and $\left\|\left[x, x^{*}\right]\right\|<\delta$ there exists a normal $y \in M_{n}$ with $\|x-y\|<\varepsilon$ and $\left\|\left[y, y^{*}\right]\right\|=0$.

Proposition 6.2.4. If $\mathfrak{A}$ has Lin's property if and only if in every ultrapower of $\mathfrak{A}$ normal elements have normal representative sequences.

Proof. Suppose $\mathfrak{A}$ does not have Lin's property, then there is an $\varepsilon_{0}>0$ so that for any $n \in \mathbb{N}$, there is an $x_{n} \in \mathfrak{A}$ with $\left\|x_{n}\right\| \leq 1$ and $\left\|\left[x_{n}, x_{n}^{*}\right]\right\| \leq 1 / n$, but for all normal $y \in \mathfrak{A},\left\|x_{n}-y\right\| \geq \varepsilon_{0}$. Let $\mathcal{U}$ be a free ultrafilter on $\mathbb{N}$. Consider $\left(x_{n}\right)_{\mathcal{U}} \in \mathfrak{A}^{\mathcal{U}}$, then

$$
\left\|\left[\left(x_{n}\right)_{\mathcal{U}},\left(x_{n}\right)_{\mathcal{U}}^{*}\right]\right\|=\lim _{\mathcal{U}}\left\|\left[x_{n}, x_{n}^{*}\right]\right\|=0
$$

So $\left(x_{n}\right)_{\mathcal{U}}$ is normal, but if $y_{n}$ is normal for all $n$ (and $\left\|y_{n}\right\|$ are bounded), then

$$
\left\|\left(x_{n}\right)_{\mathcal{U}}-\left(y_{n}\right) \mathcal{U}\right\|=\lim _{\mathcal{U}}\left\|x_{n}-y_{n}\right\| \geq \varepsilon_{0}>0
$$

So $\left(x_{n}\right)_{\mathcal{U}} \neq\left(y_{n}\right)_{\mathcal{U}}$ and $\left(x_{n}\right)_{\mathcal{U}}$ has no representative sequence of normal elements.

Suppose $\mathfrak{A}$ has Lin's property. Let $\mathcal{U}$ be an ultrafilter and $\left(x_{i}\right)_{\mathcal{U}}$ be a normal element in $\mathfrak{A}^{\mathcal{U}}$, so

$$
\left\|\left[\left(x_{i}\right)_{\mathcal{U}},\left(x_{i}\right)_{\mathcal{U}}^{*}\right]\right\|=\lim _{\mathcal{U}}\left\|\left[x_{i}, x_{i}^{*}\right]\right\|=0
$$

Via scaling, we will assume without loss of generality that $\left\|\left(x_{i}\right)_{\mathcal{U}}\right\| \leq 1$ and $\left\|x_{i}\right\| \leq 1$ for all $n$. Since $\mathfrak{A}$ has Lin's property, for every $k \in \mathbb{N}$, there is a $\delta_{k}>0$ so that for 
any $x \in \mathfrak{A}$ with $\|x\| \leq 1$ and $\left\|\left[x, x^{*}\right]\right\|<\delta_{k}$ there is an $y \in \mathfrak{A}$ so that $y$ is normal and $\|x-y\|<1 / k$.

Let $U_{k}=\left\{i:\left\|\left[x_{i}, x_{i}^{*}\right]\right\|<\delta_{k}\right\}$, then for all $k, U_{k} \in \mathcal{U}$, and for $k \leq j, U_{k} \supseteq U_{j}$. For $n \notin U_{1}$, let $y_{n}=0$. For $k \geq 1$, for each $i \in U_{k} \backslash U_{k-1}$ we know that $\left\|\left[x_{i}, x_{i}^{*}\right]\right\|<\delta_{k}$, so we can find a normal $y_{i} \in \mathfrak{A}$ with $\left\|x_{i}-y_{i}\right\|<1 / k$. For $i \in \bigcap_{k} U_{k}$ let $y_{i}=x_{i}$, which is necessarily normal. Note $\left\|y_{i}\right\| \leq\left\|x_{i}\right\|+1$ for all $i \in I$, so we can consider $\left(y_{i}\right)_{\mathcal{U}} \in \mathfrak{A}_{i}$. If $\varepsilon>0$ is given, pick $k$ so $\varepsilon>1 / k$, then for all $i \in U_{k},\left\|y_{i}-x_{i}\right\|<1 / k<\varepsilon$, so we have

$$
\left\|\left(x_{i}\right)_{\mathcal{U}}-\left(y_{i}\right)_{\mathcal{U}}\right\|=\lim _{\mathcal{U}}\left\|x_{i}-y_{i}\right\|=0
$$

Thus $\left(x_{i}\right)_{\mathcal{U}}=\left(y_{i}\right)_{\mathcal{U}}$ and we have a representative sequence of normal elements as desired.

The same proof shows that if a family of $\mathrm{C}^{*}$-algebras uniformly have Lin's property (i.e. for every $\varepsilon$, the same $\delta$ works for every algebra in the family), then every ultraproduct of elements of that family also have Lin's property. In particular,

Corollary 6.2.5. If $F_{n}$ are finite-dimensional $\mathrm{C}^{*}$-algebras and $\mathcal{U}$ is a countably incomplete ultrafilter then normal elements of $\prod_{\mathcal{U}} F_{n}$ have representative sequences of normal elements.

Question 6.2.6. What normal elements approximate the $x_{n}$ as defined in Example $5.4 .18 ?$

Proposition 6.2.7. $\mathcal{K}(\mathcal{H})$ has Lin's property. 
Proof. Let $\varepsilon>0$ be given. By Lin's Theorem there is a $\delta>0$ so if $x \in M_{n},\|x\| \leq 1+\varepsilon$, and $\left\|\left[x, x^{*}\right]\right\|<\delta$, then there is a normal matrix $y \in M_{n}$ so $\|x-y\|<\varepsilon / 2$. Suppose $x \in \mathcal{K}(\mathcal{H})$ has $\|x\| \leq 1$ and $\left\|\left[x, x^{*}\right]\right\|<\delta / 2$. Since $x \in \mathcal{K}(\mathcal{H})$, there is a finite rank operator $t$ so that

$$
\|x-t\| \leq \min \left(\frac{\varepsilon}{2}, \frac{\delta}{8+10 \varepsilon}\right)
$$

Then $\|t\| \leq 1+\frac{3}{2} \varepsilon$ and $t$ can be written in the form $\sum_{j=1}^{r} \alpha_{j} t_{\xi_{j}, \eta_{j}}$. Let $p$ be the projection onto $\operatorname{span}\left\{\xi_{1}, \ldots, \xi_{r}, \eta_{1}, \ldots, \eta_{r}\right\}$, then $p$ is a finite-dimensional projection and $p t p=t$. Thus

$\left\|\left[p t p,(p t p)^{*}\right]\right\| \leq 2(\|p t p\|+\|x\|)\|x-p t p\|+\left[x, x^{*}\right] \leq 2\left(1+\frac{3}{2} \varepsilon+1+\varepsilon\right) \frac{\delta}{8+10 \varepsilon}+\frac{\delta}{2} \leq \delta$.

Since $p \mathcal{K}(\mathcal{H}) p \cong M_{n}$ for some $n$, there is a normal $y$ with $\|p t p-y\|<\varepsilon / 2$, thus $\|y-x\|<\varepsilon$

The same proof shows that if a family of $\mathrm{C}^{*}$-algebras uniformly have Lin's property, then an inductive limit of algebras from that family (with injective maps) also has Lin's property.

Friis and Rørdam gave a useful strengthening of Lin's Theorem. First we need the following definitions:

Definition 6.2.8 (3.1 in [FR96]). For each unital $\mathrm{C}^{*}$-algebra $\mathfrak{A}$, let $R(\mathfrak{A})$ denote the set of elements $x \in \mathfrak{A}$ with the property that for no ideal $I$ of $\mathfrak{A}$ is $x+I$ one-sided and not two-sided invertible in $\mathfrak{A} / I$. A unital $\mathrm{C}^{*}$-algebra $\mathfrak{A}$ is said to have property (IR) if every element in $R(\mathfrak{A})$ belongs to the norm-closure of GL( $\mathfrak{A})$. 
Theorem 6.2.9 (4.4 in [FR96]). Any $\mathrm{C}^{*}$-algebra with property (IR) has Lin's property, in fact for every $\varepsilon>0$ there is a $\delta>0$ which will work for all $\mathrm{C}^{*}$-algebras with property (IR).

Question 6.2.10. If $\left(x_{n}\right)_{\mathcal{U}} \in \prod_{\mathcal{U}} M_{n} \cong \prod_{\mathcal{U}} \mathcal{B}\left(\mathbb{C}^{n}\right)$ is normal, is it diagonalizable when represented as an operator in $\mathcal{B}\left(\prod_{\mathcal{U}} \mathbb{C}^{n}\right)$ ? 


\section{Chapter 7}

\section{Continuous Logic}

We give a brief overview of continuous logic for $\mathrm{C}^{*}$-algebras and the main modeltheoretical results we will need.

\subsection{History}

Classical first-order logic is interested in the truth values of propositional statements. The symbols allowed in these statements vary depending the types of objects we wish to study, but to be concrete let us consider algebras. We want to be able to add, subtract, and multiply elements together. We want to be able to multiply elements by scalars. We want symbols for important elements like the zero element. We need to be able to say if elements are equal. Here is an example of a sentence:

$$
\exists x \quad(x \neq 0) \wedge\left(x^{2}=0\right)
$$


This statement is true if the algebra has a nilpotent element of order two. In general basic statements or atomic formulae are polynomials equations in several variables. We can combine those with logical connectives like "and", "or", "not", and "implies", and we can use existential and universal quantifiers. If we have quantifier over all the variables so there are no free variables the result is a sentence. We cannot quantify over arbitrary subsets (this is what "first-order" refers to), which means we cannot express many natural concepts. This logic is also finitary-sentences have finite length. This means that first-order logic cannot always distinguish non-isomorphic objects. For instance, it is possible for two objects to satisfy the same sentences but have different cardinalities. Despite all of this, the theorems that do hold like the Compactness Theorem, Gödel's Completeness Theorem, Łos' Theorem, the Keisler-Shelah Theorem, and the Löwenheim-Skolem Theorem are very powerful.

Unfortunately, classical first-order logic does not work the way we would like for continuous objects like metric spaces. This is because we have have elements which come close to satisfying an equation, without being able to exactly satisfy that equation. In order for a result like Łos' Theorem to hold for metric spaces, one must allow sentences to take on real-values rather than only being true or false (or allow for some sort of approximate satisfaction, but these turn out to be equivalent).

A version of continuous logic was introduced by Chang and Keisler in [CK66], were sentences could take on values in any compact Hausdorff space. A logic of positive bounded formulas with approximate semantics was introduced by Henson for 
Banach spaces in [Hen76] and [Hen75]. Compact abstract theories were introduced by Ben Yaacov in [BY03]. These were shown to be equivalent and expanded to the modern model theory for metric structures by Ben Yaacov, Berenstein, Henson, and Usvyatsov in [BYBHU08]. Finally this theory was specialized to $\mathrm{C}^{*}$-algebras and tracial von Neumann algebras by Farah, Hart and Sherman in [FHS13], [FHS14a] and [FHS14b]. Similar to classical first-order logic, continuous logic will not allow us to quantify over arbitrary subsets, and it will not be able to distinguish infinite cardinals.

\subsection{Language for $\mathrm{C}^{*}$-Algebras}

The language (i.e. symbols) that can be used are the norm, addition, multiplication, multiplication by any scalar $\lambda$, and the adjoint operation. Beside variables, we include the constant symbol for the zero element, and in unital $\mathrm{C}^{*}$-algebras the identity $I$. Terms are norms of $*$-polynomials in several variables. One can combine (finitely many) terms via continuous functions or "connectives", and can quantify by taking suprema and infima over the closed balls of radius $n$ to get formulae. A formula with no free variables is called a sentence. For a sentence $\varphi$, we let $\varphi^{\mathfrak{A}}$ be its evaluation in $\mathfrak{A}$, which is a real number.

Generally suprema are viewed as universal quantifiers and infima are viewed as existential quantifiers. A sentence which evaluates to zero in a $\mathrm{C}^{*}$-algebra is thought of as true in that algebra. In general $\inf _{x} \varphi(x)=0$ does not mean this infimum is 
actually achieved, of course. Note that $|\varphi|+|\psi|$ evaluates to zero if and only if both $\varphi$ and $\psi$ evaluate to zero, so this operation acts like "and". Similarly $\varphi \cdot \psi$ evaluates to zero if and only if $\varphi$ or $\psi$ evaluates to zero, so this operation acts like "or". In general negations (and implications) are problematic in this logic, since while a sentence may not evaluate to zero we cannot always bound it away from zero.

For instance, in classical first-order logic one might express that an algebra has a multiplicative unit with the sentence

$$
\exists e \quad \forall x \quad e x-x e=0 .
$$

Similarly one might express that an algebra is commutative with the sentence

$$
\forall x, y \quad x y-y x=0
$$

The analogous sentences for $\mathrm{C}^{*}$-algebras are

$$
\varphi_{u}=\inf _{\|e\| \leq 1} \sup _{\|x\| \leq 1}\|e x-x\|+\|x-e x\|
$$

and

$$
\varphi_{c}=\sup _{\|x\|,\|y\| \leq 1}\|x y-y x\| .
$$

It is clear that if a $\mathrm{C}^{*}$-algebra is unital then the first sentence evaluates to zero, and a $\mathrm{C}^{*}$-algebra is commutative if and only if the second sentence evaluates to zero. It is less clear that if $\varphi_{u}=0$ then the $\mathrm{C}^{*}$-algebra has an honest-to-goodness unit instead of a sequence of approximate units. Likewise, it is not immediately clear how small the evaluations of these sentences can be on non-unital and non-commutative $\mathrm{C}^{*}$-algebras. 
Comment: sentences are bounded by the fact the norm is sub-multiplicative and the triangle inequality. For instance,

$$
\varphi_{c}=\sup _{\|x\|,\|y\| \leq 1}\|x y-y x\| \leq \sup _{\|x\|,\|y\| \leq 1}\|x\|\|y\|+\|y\|\|x\|=2 .
$$

We will see later on that this maximum is attained. If we want to, we could always apply a linear function to ensure that the values the sentence can take on lie in the interval $[0,1]$.

Question 7.2.1. Is there a sentence $\varphi$ so for all $\lambda \in[0,1]$ there is a $\mathrm{C}^{*}$-algebra $\mathfrak{A}(\lambda)$ so that $\varphi^{\mathfrak{A}(\lambda)}=\lambda$ ?

We can at least see sentences can take on infinitely many values:

Example 7.2.2. If we include a trace in the language, the sentence

$$
\inf _{p \text { non-zero proj. }} \tau(p)
$$

takes on values $0 \cup\{1 / n \mid n \in \mathbb{N}\}$. We will see in 7.5.7 that we are allowed to quantify over the set of non-zero projections.

\subsection{Theories and Elementary Equivalence}

Definition 7.3.1. The theory of a $\mathrm{C}^{*}$-algebra $\mathfrak{A}$ is the collection of sentences which evaluate to zero: $\operatorname{Th}(\mathfrak{A})=\left\{\varphi \mid \varphi^{\mathfrak{A}}=0\right\}$.

Comment: the theory of a $\mathrm{C}^{*}$-algebra $\mathfrak{A}$ records the evaluation of every sentence $\varphi$, since $\psi=\varphi-\varphi^{\mathfrak{A}}$ will be in the theory of $\mathfrak{A}$. 
Definition 7.3.2. Two $C^{*}$-algebras $\mathfrak{A}$ and $\mathfrak{B}$ are elementarily equivalent, written $\mathfrak{A} \equiv \mathfrak{B}$ if for every first-order sentence $\varphi, \varphi^{\mathfrak{A}}=\varphi^{\mathfrak{B}}$. In other words, $\operatorname{Th}(\mathfrak{A})=\operatorname{Th}(\mathfrak{B})$.

Elementarily equivalent $\mathrm{C}^{*}$-algebras are indistinguishable by continuous logic. This is in general a coarser relation than $*$-isomorphism:

Example 7.3.3 (6.1 in [FHS14a], 5.5 in [GH01]). Let $\mathfrak{A}=M_{2^{\infty}}$ be the CAR algebra, and let $\mathcal{U}$ be a free ultrafilter on the natural numbers. Then $\mathfrak{A}$ is separable and simple, but it is elementarily equivalent to $\mathfrak{A}^{\mathcal{U}}$ which is neither (see 7.4.1). To see $\mathfrak{A}^{\mathcal{U}}$ is non-simple, consider the closed, non-empty ideal $\left\{\left(a_{n}\right)_{\mathcal{U}}: \lim _{\mathcal{U}} \tau\left(a_{n}^{*} a_{n}\right)=0\right\}$. This corresponds to the fact the trace $\tau_{\mathcal{U}}$ on $(\mathfrak{A})^{\mathcal{U}}$ given by $\tau_{\mathcal{U}}\left(\left(a_{n}\right)_{\mathcal{U}}\right)=\lim _{\mathcal{U}} \tau\left(a_{n}\right)$ is not faithful.

However, there are cases where elementary equivalence and isomorphism coincide:

Theorem 7.3.4 $\left(\left[\mathrm{CCF}^{+} 14\right]\right)$. For unital separable UHF algebras, isomorphism is the same as elementary equivalence.

The idea of the proof is that the formulae defining matrix units are weakly stable, so the theory determines the supernatural number associated to the algebra.

Definition 7.3.5. We say $\mathfrak{A}$ is an elementary $C^{*}$-subalgebra of $\mathfrak{B}$ if for all formulae $\varphi(\vec{x})$, and all tuples $\vec{a}$ from $\mathfrak{A}^{n}, \varphi(\vec{a})^{\mathfrak{A}}=\varphi(\vec{a})^{\mathfrak{B}}$.

Note that $\mathfrak{A} \preceq \mathfrak{B}$ implies $\mathfrak{A} \equiv \mathfrak{B}$. However, in general, being an elementary submodel is stronger than being an elementary equivalent $\mathrm{C}^{*}$-subalgebra. The following result gives a useful test for being an elementary submodel: 
Theorem 7.3.6 (Continuous Tarski-Vaught Criterion, 4.5 in [BYBHU08]). Let $\mathfrak{A}, \mathfrak{B}$ be $\mathrm{C}^{*}$-algebras with $\mathfrak{A} \subseteq \mathfrak{B}$ (if $\mathfrak{A}$ and $\mathfrak{B}$ are unital we require them to have the same unit). Then the following are equivalent:

1. $\mathfrak{A}$ is an elementary $\mathrm{C}^{*}$-subalgebra of $\mathfrak{B}$.

2. For every formula $\psi(\vec{x}, y)$, and all tuples $\vec{a}$ from $\mathfrak{A}$, if $\inf _{b \in \mathfrak{B}_{\leq 1}} \psi(\vec{a}, b)=0$, then for all $\varepsilon>0$, there is an $a \in \mathfrak{A}_{\leq 1}$ so that $\psi(\vec{a}, a)<\varepsilon$. That is, for all formulae $\psi(\vec{x}, y)$ and all tuples $\vec{a}$ from $\mathfrak{A}$,

$$
\inf _{b \in \mathcal{B}_{\leq 1}} \psi(\vec{a}, b)=\inf _{a \in \mathfrak{A}_{\leq 1}} \psi(\vec{a}, a)
$$

Here is another version:

Theorem 7.3.7 (Automorphism Tarski-Vaught, 2.1 in [HRR07]). Let $\mathfrak{A}, \mathfrak{B}$ be $\mathrm{C}^{*}$ algebras with $\mathfrak{A} \subseteq \mathfrak{B}$, and for each finite system $\left(a_{1}, \ldots, a_{n}\right)$ of $\mathfrak{A}$, and $b \in \mathfrak{B}$, and each $\varepsilon>0$, there is an automorphism $T$ of $\mathfrak{B}$ an an element $a \in \mathfrak{A}$ with

$$
\left\|T a_{i}-a_{i}\right\|<\varepsilon \quad \text { and } \quad\|T b-a\|<\varepsilon .
$$

Then $\mathfrak{A}$ is an elementary substructure of $\mathfrak{B}$.

We use the automorphism Tarski-Vaught theorem to show that the algebras $\mathcal{K}(\mathcal{H})$ with $\mathcal{H}$ is infinite-dimensional are all elementarily equivalent:

Theorem 7.3.8. If $\mathcal{H}_{1}, \mathcal{H}_{2}$ are infinite-dimensional Hilbert spaces with $\mathcal{H}_{1} \subseteq \mathcal{H}_{2}$, then $\mathcal{K}\left(\mathcal{H}_{1}\right)$ is an elementary $\mathrm{C}^{*}$-subalgebra of $\mathcal{K}\left(\mathcal{H}_{2}\right)$. 
Proof. We will use the previous theorem. The idea is that operators in $\mathcal{K}(\mathcal{H})$ are well-approximated by finite-rank operators, and since the Hilbert spaces are infinitedimensional, we can conjugate by a unitary to put the cokernels and ranges of those operators into the smaller Hilbert space $\mathcal{H}_{1}$. Fix $a_{1}, \ldots, a_{n} \in \mathcal{K}\left(\mathcal{H}_{1}\right), b \in \mathcal{K}\left(\mathcal{H}_{2}\right)$, and $\varepsilon>0$ be given. Take finite-rank operators $a_{i}^{\prime} \in \mathcal{K}\left(\mathcal{H}_{1}\right)$ so $\left\|a_{i}-a_{i}^{\prime}\right\|<\varepsilon / 2$ and a finite rank operator $b^{\prime} \in \mathcal{K}\left(\mathcal{H}_{2}\right)$ with $\left\|b-b^{\prime}\right\|<\varepsilon$. Pick an orthonormal system $\xi_{1}, \ldots, \xi_{m}$ of $M_{0}=\operatorname{span}_{1 \leq i \leq n}\left(a_{i}^{\prime} \mathcal{H},\left(a_{i}^{\prime}\right)^{*} \mathcal{H}\right) \subseteq \mathcal{H}_{1}$, and expand this (if necessary) to an orthonormal system of $M_{2}=\operatorname{span}\left(M_{0}, b_{0}^{\prime} \mathcal{H},\left(b_{0}^{\prime}\right)^{*} \mathcal{H}\right)$ of $\mathcal{H}_{2}$ by appending $\eta_{m+1}, \ldots, \eta_{\ell}$, where the $\eta_{j} \in \mathcal{H}_{2} \ominus \mathcal{H}_{1}$. Finally expand this to an orthonormal basis of $\mathcal{H}_{2}$ by appending $\eta_{n}$ for $n>\ell$. Since $\mathcal{H}_{1}$ is infinite-dimensional, pick $\xi_{i+1}, \ldots, \xi_{\ell} \in \mathcal{H}_{1}$ so $\xi_{1}, \ldots, \xi_{\ell}$ is an orthonormal system in $\mathcal{H}_{1}$. Let $M_{1}=\operatorname{span}\left(\xi_{1}, \ldots, \xi_{\ell}\right)$. Expand this to an orthnormal basis of $\mathcal{H}_{2}$ by appending $\xi_{n}$ for $n>\ell$. Consider the unitary $u: \mathcal{H}_{2} \rightarrow \mathcal{H}_{2}$ which sends $M_{1}$ to $M_{2}$, defined by $u \xi_{i}=\xi_{i}$ for $1 \leq i \leq m, u \eta_{j}=\xi_{j}$ for $m+1 \leq j \leq \ell$, and $u \eta_{n}=\xi_{n}$ for $n>\ell$. The automorphism $T$ defined by $T x=u^{*} x u$ fixes the $a_{i}^{\prime}$ and $T b^{\prime}$ is in $\mathcal{K}\left(\mathcal{H}_{1}\right)$, so

$$
\left\|T a_{i}-a_{i}\right\| \leq\left\|T a_{i}-T a_{i}^{\prime}\right\|+\left\|T a_{i}^{\prime}-a_{i}^{\prime}\right\|+\left\|a_{i}^{\prime}-a_{i}\right\|<\varepsilon
$$

and

$$
\left\|T b-T b^{\prime}\right\|<\left\|b-b^{\prime}\right\|<\varepsilon .
$$




\subsection{Continuous Model Theory}

The following is sometimes referred to as the Fundamental Theorem of Ultraproducts:

Theorem 7.4.1 (Łos' Theorem, 5.4 in [BYBHU08]). Let $\mathcal{U}$ be an ultrafilter. Then for any sentence $\varphi$,

$$
\varphi^{\prod_{\mathcal{U}} \mathfrak{A}_{i}}=\lim _{\mathcal{U}} \varphi^{\mathfrak{A}_{i}}
$$

In particular, $\mathfrak{A}$ is elementarily equivalent to all of its ultrapowers: $\mathfrak{A} \equiv \mathfrak{A}^{\mathcal{U}}$ for all ultrafilters $\mathcal{U}$.

In fact for a formula $\varphi, \vec{a}_{k}=\left(a_{i}^{(k)}\right)_{\mathcal{U}}$ for $1 \leq k \leq n$,

$$
\varphi\left(\vec{a}_{1}, \ldots, \vec{a}_{n}\right)=\lim _{\mathcal{U}} \varphi^{\mathfrak{A}_{i}}\left(a_{i}^{(1)}, \ldots, a_{i}^{(n)}\right) .
$$

In particular, the image of $\mathfrak{A}$ in $\mathfrak{A}^{\mathcal{U}}$ under the canonical embedding $a \mapsto(a)_{\mathcal{U}}$ is an elementary substructure.

Łos' theorem says that ultraproducts are logical limits of the components.

The following notion shows that ultrapowers are also logical completions - intuitively if one can approximately satisfy a collection of sentences then one can exactly satisfy all of the sentences.

Definition 7.4 .2 . For a cardinal $\kappa$ we say that a $\mathrm{C}^{*}$-algebra $\mathfrak{A}$ is $\kappa$-saturated if the following holds. Let $A$ be a subset of $\mathfrak{A}$ with cardinality $<\kappa$. Add to the language a constant symbol for each element of $A$. Let $\left\{\varphi_{i}(\vec{x})\right\}$ be a set of formulae in the augmented language (in other words: a collection of formulae with fewer than $\kappa$ 
parameters from $\mathfrak{A})$ that is approximately satisfied, i.e. for any finite subset $\left\{\varphi_{i_{k}}\right\}$ and $\varepsilon>0$ there is a tuple $\vec{a} \in \mathfrak{A}^{n}$ such that $\max _{k}\left|\varphi_{i_{k}}(\vec{a})\right|<\varepsilon$. Then there is a tuple in $\mathfrak{A}^{n}$ which exactly satisfies $\varphi_{i}(\vec{x})=0$.

See [She09] for some general philosophy and specific instances of this phenomenon. For more information on saturation of $\mathrm{C}^{*}$-algebras, see [EV15]. Many of the arguments in Chapter 5 rely on the saturation of ultraproducts.

Theorem 7.4.3 (7.6, 7.10 in [BYBHU08], 4.11 in [FHS14a]). Let $\mathfrak{A}$ be a $\mathrm{C}^{*}$-algebra.

1. If $\mathcal{U}$ is a free ultrafilter on $\mathbb{N}$ then $\mathfrak{A}^{\mathcal{U}}$ is $\aleph_{1}$-saturated. Ultraproducts with respect to $\mathcal{U}$ are also $\aleph_{1}$ saturated.

2. For any cardinal $\kappa, \mathfrak{A}$ has an ultrapower that is $\kappa$-saturated.

The second statement contains the so-called upward Lowenheim-Skolem theorem that any $\mathrm{C}^{*}$-algebra is elementarily equivalent to $\mathrm{C}^{*}$-algebras of arbitrarily large cardinality. One can also find smaller elementarily equivalent models within reason - the language gives a minimal threshold required:

Definition 7.4.4. The density character of a set in a topological space, denoted $\operatorname{dens}(\cdot)$, is the minimal cardinality of a dense set.

Theorem 7.4.5 (Downward Lowenheim-Skolem, 7.3 in [BYBHU08]). Let $X$ be a subset of a $\mathrm{C}^{*}$-algebra $\mathfrak{A}$. Then there is an elementary $\mathrm{C}^{*}$-subalgebra $\mathfrak{A}_{0}$ of $\mathfrak{A}$ with $X \subseteq \mathfrak{A}_{0}$, and dens $\left(\mathfrak{A}_{0}\right) \leq \operatorname{dens}(X)+\aleph_{0}$. In particular taking $X=\emptyset$ gives us that every $C^{*}$-algebra is elementarily equivalent to a separable $\mathrm{C}^{*}$-subalgebra. 
This type of construction for $\mathrm{C}^{*}$-algebras was used by Blackadar, see Proposition 2.2 in [Bla78] and [PV13].

The following famous result completes the link between ultrapowers and continuous logic:

Theorem 7.4.6 (Keisler-Shelah, 5.7 in [BYBHU08]). Two $\mathrm{C}^{*}$-algebras are elementarily equivalent if and only if they have isomorphic ultrapowers (with respect to the same ultrafilter, even). That is, $\mathfrak{A} \equiv \mathfrak{B}$ if and only if there is some ultrafilter $\mathcal{U}$ so that $\mathfrak{A}^{\mathcal{U}} \cong \mathfrak{B}^{\mathcal{U}}$

Proposition 7.4.7. If $\mathfrak{A}_{1} \equiv \mathfrak{A}_{2}$ and $\mathfrak{B}_{1} \equiv \mathfrak{B}_{2}$ then $\mathfrak{A}_{1} \oplus \mathfrak{B}_{1} \equiv \mathfrak{A}_{2} \oplus \mathfrak{B}_{2}$.

Proof. By the Keisler-Shelah theorem, there are ultrafilters $\mathcal{U}$ and $\mathcal{V}$ so that

$$
\mathfrak{A}_{1}^{\mathcal{U}} \cong \mathfrak{A}_{2}^{\mathcal{U}} \quad \text { and } \quad\left(\mathfrak{B}_{1}^{\mathcal{U}}\right)^{\mathcal{V}} \cong\left(\mathfrak{B}_{2}^{\mathcal{U}}\right)^{\mathcal{V}}
$$

Then since ultrapowers and (finite) direct sums commute,

$$
\begin{aligned}
\left(\mathfrak{A}_{1} \oplus \mathfrak{B}_{1}\right)^{\mathcal{U} \times \mathcal{V}} \cong\left(\left(\mathfrak{A}_{1} \oplus \mathfrak{B}_{1}\right)^{\mathcal{U}}\right)^{\mathcal{V}} & \cong\left(\mathfrak{A}_{1}^{\mathcal{U}}\right)^{\mathcal{V}} \oplus\left(\mathfrak{B}_{1}^{\mathcal{U}}\right)^{\mathcal{V}} \\
& \cong\left(\mathfrak{A}_{2}^{\mathcal{U}}\right)^{\mathcal{V}} \oplus\left(\mathfrak{B}_{2}^{\mathcal{U}}\right)^{\mathcal{V}} \cong\left(\mathfrak{A}_{2} \oplus \mathfrak{B}_{2}\right)^{\mathcal{U} \times \mathcal{V}}
\end{aligned}
$$

\subsection{Definability}

In this section we try to elucidate the connections between the the notions of definability and weakly-stable relations.

The following is a useful shorthand when writing sentences: 
Definition 7.5.1 (3.13 in [BYBHU08]). $\varphi-\psi \cdot=\max (0, \varphi-\psi)$.

Observe that $\varphi \dot{-} \psi=0$ if and only if $\varphi \leq \psi$.

The next result shows how implications work in continuous logic:

Proposition 7.5.2 (7.15 in [BYBHU08]). The following are equivalent:

1. For all $\mathrm{C}^{*}$-algebras $\mathfrak{A}$, and all $\vec{a} \in \mathfrak{A}^{n}$, if $\varphi^{\mathfrak{A}}(\vec{a})=0$ then $\psi^{\mathfrak{A}}(\vec{a})=0$.

2. For all $\varepsilon>0$ there exist $\delta>0$ such that

$$
\sup _{x} \min (\delta \dot{-} \varphi(x), \psi(x) \dot{-} \varepsilon)=0 .
$$

3. There exists an increasing, continuous function $\alpha:[0,1] \rightarrow[0,1]$ with $\alpha(0)=0$ such that

$$
\sup _{x}(\psi(x)-\alpha(\varphi(x))=0 .
$$

Definition 7.5.3 $\left(9.1,9.16\right.$, in [BYBHU08]). A predicate $P: \mathfrak{A}^{n} \rightarrow \mathbb{R}$ is definable if for every $k \geq 1$ there is a $n$-ary formula $\varphi_{k}(\vec{x})$ such that

$$
\sup _{\vec{x} \in \mathfrak{A}_{\leq 1}^{n}}\left|P(\vec{x})-\varphi_{k}(\vec{x})\right| \leq \frac{1}{k} .
$$

A subset $X$ of $\mathfrak{A}^{n}$ is definable if the function $D_{X}(x)=\operatorname{dist}(x, X)$ is a definable predicate.

Definable sets are important because we can quantify over definable subsets:

Theorem 7.5.4 (9.17 in [BYBHU08]). For a closed set $D \subseteq \mathfrak{A}^{n}$, the following are equivalent: 
1. $D$ is definable in $\mathfrak{A}$.

2. For any predicate $P: \mathfrak{A}^{m} \times \mathfrak{A}^{n} \rightarrow[0,1]$ that is definable in $\mathfrak{A}$, the predicate $Q: \mathfrak{A}^{m} \rightarrow[0,1]$ defined by

$$
Q(x)=\inf \{P(\vec{x}, \vec{y}) \mid \vec{y} \in D\}
$$

is definable in $\mathfrak{A}$.

Proposition 7.5.5 (9.19 in [BYBHU08]). For a closed set $D \subseteq \mathfrak{A}^{n}$, the following are equivalent:

1. $D$ is definable in $\mathfrak{A}$.

2. There is a predicate $P: \mathfrak{A}^{n} \rightarrow[0,1]$, definable in $\mathfrak{A}$ i.e. it is a uniform limit of a sequence of formulae $\varphi_{k}(x)$ :

$$
\forall \varepsilon>0 \quad \exists N \quad \forall k \geq N \quad \forall x \in \mathfrak{A}^{n} \quad\left(\left|\varphi_{k}^{\mathfrak{A}}(x)-P(x)\right| \leq \varepsilon\right)
$$

such that $P(x)=0$ for all $x \in D$, and

$$
\forall \varepsilon>0 \quad \exists \delta>0 \quad \forall x \in \mathfrak{A}^{n} \quad(P(x) \leq \delta \Rightarrow \operatorname{dist}(x, D) \leq \varepsilon)
$$

3. There is a sequence of formulae $\varphi_{m}$ and a sequence of positive real numbers $\left\{\delta_{m}\right\}$ such that for all $m \geq 1$ and $x \in \mathfrak{A}^{n}$,

$$
x \in D \Rightarrow \varphi_{m}^{\mathfrak{A}}(x)=0
$$

and

$$
\varphi_{m}^{\mathfrak{A}}(x) \leq \delta_{m} \rightarrow \operatorname{dist}(x, D) \leq \frac{1}{m} .
$$


Definable sets are related to the following notion:

Definition 7.5.6. An $n$-ary formula $\varphi(\vec{x})$ is weakly stable if for all $\varepsilon>0$ there is a $\delta>0$ such that for every $\mathrm{C}^{*}$-algebra $\mathfrak{A}$ and every $\vec{a} \in \mathfrak{A}^{n}$ with $|\varphi(\vec{a})|<\delta$ there is a $\vec{a}^{\prime} \in \mathfrak{A}^{n}$ such that $\left\|a_{i}-a_{i}^{\prime}\right\|<\varepsilon$ for $1 \leq i \leq n$ and $\varphi\left(\vec{a}^{\prime}\right)=0$.

For more information about weakly stable relations, see Loring's book [Lor97]. Weakly stable relations are very important because of the following result:

Corollary 7.5.7 (e.g. 3.2.4 in $\left[\mathrm{FHL}^{+} 16\right]$ ). The zero sets of weakly-stable formulae are definable sets, and we can quantify over those sets.

In particular, we have seen in 3.2.1 that the self-adjoint elements (the zero set of $\left.\left\|x-x^{*}\right\|\right)$, the positive elements $\left(\inf _{y}\left\|y^{*} y-x\right\|\right)$, the projections $\left(\left\|p-p^{2}\right\|+\left\|p-p^{*}\right\|\right)$, the isometries and partial isometries $\left(\left\|x^{*} x-I\right\|\right.$ and $\left\|x^{*} x-\left(x^{*} x\right)^{2}\right\|$ respectively), and the unitary elements $\left(\left\|x^{*} x-I\right\|+\left\|x x^{*}-I\right\|\right)$ are definable, and we can quantify over those sets. Conversely, we have seen that the normal $\left(\left\|x x^{*}-x^{*} x\right\|\right)$, invertible $\left(\inf _{y}\|x y-I\|+\|y x-I\|\right)$ are not in general definable sets, so in general we cannot quantify over those sets. Likewise we will see the center of a $\mathrm{C}^{*}$-algebra $\left(\sup _{y} \| x y-\right.$ $y x \|)$ is not in general a definable set, see Example 11.4.8. See 3.2.5 in $\left[\mathrm{FHL}^{+} 16\right]$.

It is easy to explicitly quantify over positive and self-adjoint elements:

$$
\begin{aligned}
& \sup _{x \geq 0,\|x\| \leq 1} \varphi(x)=\sup _{\|a\| \leq 1} \varphi\left(a^{*} a\right) \\
& \sup _{x \text { sa },\|x\| \leq 1} \varphi(x)=\sup _{\|a\| \leq 1} \varphi\left(\frac{a^{*}+a}{2}\right)
\end{aligned}
$$


Note that we can use exp and $|x|=\left(x^{*} x\right)^{1 / 2}$ in our language (they are definable predicates) by the continuous functional calculus. $\exp (\lambda)=\sum_{n \geq 0} \lambda^{n} / n$ ! is uniformly continuous on the unit ball, so it is a uniform limit of polynomials, and similarly $\sqrt{\lambda}$ is a uniform limit of polynomials on the interval $[0,1]$.

It is also known that we can quantify over certain compact subsets of the complex numbers. For instance:

$$
\begin{gathered}
\sup _{0 \leq \lambda \leq 1} \varphi(\lambda)=\sup _{\|x\| \leq 1} \varphi(\|x\|) \\
\sup _{|\lambda| \leq 1} \varphi(\lambda)=\sup _{\|x\|,\|y\| \leq 1} \varphi(\|x\| \exp (2 \pi i\|y\|))
\end{gathered}
$$

There are many other interesting topics related to logic and model theory for metric structures. A good overview of using set theoretic axioms to prove results in operator algebras is [Wea07]. The interest was spurred by results like the consistency of a counter-example to Naimark's problem [AW04] and the proof of the independence of the existence of outer automorphisms on the Calkin algebra, see [PW07], [Far11] and [FMS13]. A good overview for applying logic to operator algebras is [Far14]. For existentially-closed $\mathrm{II}_{1}$ factors see [FGHS16]. For EF games in continuous logic, see [GS15a]. For omitting types in continuous logic, see $[\mathrm{FM} 14]$ and $\left[\mathrm{CCF}^{+} 14\right]$. For quantifier elimination in $\mathrm{C}^{*}$-algebras see [EFKV15]. Many useful insights are available in $\left[\mathrm{FHL}^{+} 16\right]$. Model Theory of $\mathrm{C}^{*}$-algebras has connections to several major problems such the the QWEP conjecture [Gol15] and the Kirchberg Embedding problem [GS15b]. Descriptive set theory [FTT13] and Fraïssé limits of $\mathrm{C}^{*}$-algebras $\left[\mathrm{EFH}^{+} 14\right]$ have also been studied. 


\section{Chapter 8}

\section{Particular Preserved Properties}

We catalogue some axiomatizable properties of $\mathrm{C}^{*}$-algebras relevant to pseudocompact $\mathrm{C}^{*}$-algebras. This list is by no means comprehensive. Most of these results are known, although some of our methods are new. A more complete list of known properties appears in Section 3 of $\left[\mathrm{FHL}^{+} 16\right]$.

\subsection{Axiomatizable and Local Classes}

Definition 8.1.1. A (closed) condition is of the form $\varphi \leq r$ for a sentence $\varphi$ and $r \in \mathbb{R}$.

Definition 8.1.2. A class of $\mathrm{C}^{*}$-algebras is axiomatizable if there is a collection of conditions $\Sigma$ so that $\mathcal{C}$ is the collection of $\mathrm{C}^{*}$-algebras satisfying those conditions. We say $\Sigma$ is a set of axioms for that class. 
We say that a property is axiomatizable if the class of $\mathrm{C}^{*}$-algebras which enjoy that property is axiomatizable. The next result allows us to show that properties are axiomatizable without explicitly finding the axioms. We say a property is closed under ultraroots if whenever $\mathfrak{A}^{\mathcal{U}}$ has that property, $\mathfrak{A}$ also has that property.

Proposition 8.1.3 (5.14 in [BYBHU08]). A class of $\mathrm{C}^{*}$-algebras is axiomatizable if and only if it is closed under $*$-isomorphism, ultraproducts, and ultraroots.

A class being closed under ultraroots is the same as the complement of that class being closed under ultrapowers.

A slightly weaker notion is due to Henson [Hen76]:

Definition 8.1.4. A class of $\mathrm{C}^{*}$-algebras is local if if it closed under $*$-isomorphism, ultrapowers, and ultraroots. This is equivalent to being closed under elementary equivalence by Łos' theorem.

Comment: If one considers the class of $\mathrm{C}^{*}$-algebras modulo elementary equivalence, then this space has a natural Hausdorff topology generated by closed sets $C_{\varphi, r}=\left\{\mathfrak{A}: \varphi^{\mathfrak{A}} \leq r\right\}$. Then local classes correspond to arbitrary subsets of this class, and axiomatizable classes correspond to closed sets. See Section 3 of [FHS14b].

\subsection{Non-Axiomatizable and Non-Local Classes}

Example 8.2.1 (e.g. 3.2.1 in [FHS14b]). The collection of finite-dimensional C*algebras is a local class, see 2.6.1. It is not an axiomatizable class, see 2.6.2. 
Example 8.2.2 (e.g. 3.2.1 in [FHS14b]). The collection of infinite-dimensional C*algebras is an axiomatizable class. It is easy to see that being infinite-dimensional is stable under $*$-isomorphism, ultraproducts, and ultraroots. Alternatively, one can write sentences $\varphi_{n}$ stating that there are $n$ points in the unit ball of an algebra which are distance at least 1 apart. Since the unit ball of a $\mathrm{C}^{*}$-algebra is compact if and only if the algebra is finite-dimensional, $\varphi_{n}^{\mathfrak{A}}$ will be satisfied for all $n$ if and only if the algebra is infinite-dimensional.

Example 8.2.3. Example 5.4.7 shows the collection of elementary $\mathrm{C}^{*}$-algebras (those $\mathrm{C}^{*}$-algebras $*$-isomorphic to some $\left.\mathcal{K}(\mathcal{H})\right)$ is not an axiomatizable or local class.

Example 8.2.4 7.3.3 shows that the collection of UHF $\mathrm{C}^{*}$-algebras is not an axiomatizable or local class. The separable $\mathrm{C}^{*}$-algbras are not an axiomatizable class. The simple $\mathrm{C}^{*}$-algebras are not an axiomatizable or local class. The non-simple $\mathrm{C}^{*}$ algebras are not an axiomatizable or local class. $\mathrm{C}^{*}$-algebras with a unique faithful trace are not an axiomatizable or local class.

Proposition 8.2.5. Let $\mathcal{U}$ be an ultrafilter on $I$, and for all $i \in I$ suppose $\mathfrak{A}_{i}$ is a $\mathrm{C}^{*}$-algebra. If $\mathfrak{A}_{i}$ are unital and non-simple, then $\prod_{\mathcal{U}} \mathfrak{A}_{i}$ is non-simple.

Proof. Let $J_{i}$ be a proper ideal in $\mathfrak{A}_{i}$ and $I_{i}$ be the unit of $\mathfrak{A}_{i}$. Then for all $j \in J_{i}$, $\left\|j-I_{i}\right\| \geq 1$, since $\left\|j-I_{i}\right\|<1$ would imply that $j$ is invertible. Thus $\prod_{\mathcal{U}} J_{i}$ is a proper ideal of $\prod_{\mathcal{U}} A_{i}$.

The following famous result is very useful for us. It allows us to find elements of 
a $\mathrm{C}^{*}$-algebra which behave like specific matrices:

Theorem 8.2.6 (Kadison Transitivity Theorem, [Kad57]). Let $\pi$ be an irreducible representation of a $\mathrm{C}^{*}$-algebra $\mathfrak{A}$ on a Hilbert space $\mathcal{H}$. Suppose that $t \in \mathcal{B}(\mathcal{H}), \mathcal{K}$ is a finite-dimensional subspace of $\mathcal{H}$, and let $\varepsilon>0$ be given. Then there is an $a \in \mathfrak{A}$ such that

$$
\left.\pi(a)\right|_{\mathcal{K}}=\left.\pi(t)\right|_{\mathcal{K}} \quad \text { and } \quad\|a\| \leq\|t\|+\varepsilon
$$

\subsection{Unital $\mathrm{C}^{*}$-Algebras Are Axiomatizable}

Lemma 8.3.1. Suppose $\mathfrak{A}$ is a non-unital $\mathrm{C}^{*}$-algebra. Then for any projection $p$ of $\mathfrak{A}$, and $\varepsilon>0$, there is an element $x$ of $\mathfrak{A}$ such that $\|x\| \leq 1+\varepsilon$, and $\|p x-x\| \geq 1$. Proof. Let $\pi: \mathfrak{A} \rightarrow \mathcal{B}(\mathcal{H})$ be an irreducible representation of $\mathfrak{A}$ so $\pi(p) \neq \operatorname{id}_{\mathcal{H}}$ (otherwise, let $\pi$ be the direct sum of all irreducible representations of $\mathfrak{A}$, then $\pi$ is a faithful representation of $\mathfrak{A}$ and $\pi(p)$ acts as the identity on $\pi(\mathfrak{A})$, a contradiction). In particular there is a unit vector $\xi \in \mathcal{H}$ so $\pi(p) \xi=0$, and there is a rank one projection $q \in B(\mathcal{H})$ so that $q \xi=\xi$. By Kadison transitivity, there is an element $x \in \mathfrak{A}$ so that $\|x\| \leq 1+\varepsilon$ and $\pi(x) \xi=q \xi=\xi$. Thus

$$
\|p x-x\| \geq\|\pi(p x-x)\| \geq\|[\pi(p) \pi(x)-\pi(x)] \xi\|=\|\xi\|=1 .
$$

The following result is known:

Theorem 8.3.2. Let $\mathcal{U}$ be an ultrafilter on $I$, and for all $i \in I$ suppose $\mathfrak{A}_{i}$ is a $\mathrm{C}^{*}$-algebra. Then $\prod_{\mathcal{U}} \mathfrak{A}_{i}$ is unital if and only if $\left\{i \mid \mathfrak{A}_{i}\right.$ is unital $\} \in \mathcal{U}$. 
Proof. $(\Leftarrow)$ : This is clear.

$(\Rightarrow)$ : Suppose $N=\left\{i \mid \mathfrak{A}_{i}\right.$ is not unital $\} \in \mathcal{U}$. Since any potential unit in $\prod_{\mathcal{U}} \mathfrak{A}_{i}$ is a projection, let $\left(e_{i}\right)_{\mathcal{U}} \in \prod_{\mathcal{U}} \mathfrak{A}_{i}$ be a projection. Without loss of generality we can assume $\left(e_{i}\right)_{\mathcal{U}}=\left(p_{i}\right)_{\mathcal{U}}$ where the $p_{i}$ are projections. For $i \in N, p_{i}$ is not the identity, so by the previous lemma we can find $x_{i}$ so $\left\|x_{i}\right\| \leq 2$ and $\left\|p_{i} x_{i}-x_{i}\right\| \geq 1$. Then the element $\left(x_{i}\right)_{\mathcal{U}} \in \prod_{\mathcal{U}} \mathfrak{A}_{i}$ is well-defined, and

$$
\left\|\left(p_{i} x_{i}\right)_{\mathcal{U}}-\left(x_{i}\right)_{\mathcal{U}}\right\|_{\mathcal{U}}=\lim _{\mathcal{U}}\left\|p_{i} x_{i}-x_{i}\right\| \geq 1
$$

So $\left(p_{i}\right)_{\mathcal{U}}\left(x_{i}\right)_{\mathcal{U}} \neq\left(x_{i}\right)_{\mathcal{U}}$, so $\left(e_{i}\right)_{\mathcal{U}}=\left(p_{i}\right)_{\mathcal{U}}$ is not a unit, and $\prod_{\mathcal{U}} \mathfrak{A}_{i}$ is not unital as claimed.

Comment: We know the axiom for $\mathfrak{A}$ being unital:

$$
\varphi_{u}=\inf _{\|e\| \leq 1} \sup _{\|x\| \leq 1}\|e x-x\|+\|x-e x\|=0
$$

We have shown an axiom for $\mathfrak{A}$ being non-unital:

$$
\varphi_{u}=\inf _{\substack{\|p\| \leq 1 \\ p \text { self-adjoint }}} \sup _{\|x\| \leq 1}\|p x-x\| \geq 1
$$

Thus the unital and non-unital $\mathrm{C}^{*}$-algebras are axiomatizable classes.

In other words, an element which almost behaves like an identity element is the identity element. This is somewhat surprising if we recall the following result:

Theorem 8.3.3 (Approximate Units, see I.4.8 in [Dav96] e.g.). If $\mathfrak{A}$ is a separable $\mathrm{C}^{*}$-algebra, there is an increasing sequence of positive contractions $\left(e_{n}\right)_{n \in \mathbb{N}}$ so that 
for all $x \in \mathfrak{A}$,

$$
\lim _{n \rightarrow \infty}\left\|e_{n} x-x\right\|=0=\lim _{n \rightarrow \infty}\left\|x e_{n}-x\right\|
$$

Non-separable $\mathrm{C}^{*}$-algebras also have approximate units, but they are typically a net of increasing positive contractions instead of a sequence.

Proposition 8.3.4. Let $\mathfrak{A}$ be a separable non-unital $\mathrm{C}^{*}$-algebra, $\mathcal{U}$ a free ultrafilter on $\mathbb{N}$. Then the unitization $\mathfrak{A}^{\sim}$ of $\mathfrak{A}$ embeds into $\mathfrak{A}^{\mathcal{U}}$, and the inclusion of $\mathfrak{A}$ into $\mathfrak{A}^{\sim}$ into $\mathfrak{A}^{\mathcal{U}}$ is the usual diagonal embedding $x \mapsto(x)_{\mathcal{U}}$.

Proof. Let $\left(e_{n}\right)_{n \in \mathbb{N}}$ be an approximate unit for $\mathfrak{A}$. Then $\left(e_{n}\right)_{\mathcal{U}}$ acts like a multiplicative identity on all $(x)_{\mathcal{U}}$ for all $x \in \mathfrak{A}$. Thus the $\mathrm{C}^{*}$-algebra generated by $\left\{(x)_{\mathcal{U}} \mid x \in \mathfrak{A}\right\}$ and $\left(e_{n}\right)_{\mathcal{U}}$ is isomorphic to the unitization $\mathfrak{A}^{\sim}$ of $\mathfrak{A}$.

Of course there is an analogous result for non-separable $\mathrm{C}^{*}$-algebras, but $\mathcal{U}$ needs to be a free ultrafilter on the index set of the net. Note however that if $\mathfrak{A}$ is not unital, then $\left(e_{n}\right)_{\mathcal{U}}$ is not a unit for $\mathfrak{A}^{\mathcal{U}}$.

\subsection{Commutative $\mathrm{C}^{*}$-Algebras Are Axiomatizable}

Lemma 8.4.1. Suppose $\mathfrak{A}$ is a non-commutative $\mathrm{C}^{*}$-algebra. Then for all $\varepsilon>0$, there are elements $a, b$ of $\mathfrak{A}$ so that $\|a\|,\|b\| \leq 1+\varepsilon$ and $\|[a, b]\| \geq 2$.

Proof. Since $\mathfrak{A}$ is non-commutative, not every irreducible representation of $\mathfrak{A}$ is onedimensional. Let $\pi: \mathfrak{A} \rightarrow \mathcal{B}(\mathcal{H})$ be an irreducible representation of $\mathfrak{A}$ where $\mathcal{H}$ is 
at least two-dimensional. Let $\xi, \eta$ be orthogonal unit vectors in $\mathcal{H}$, and let $\mathcal{K}=$ $\operatorname{span}(\xi, \eta)$. Then there are operators $t_{1}, t_{2} \in \mathcal{B}(\mathcal{H})$ so that

$$
t_{1} \xi=-\eta \quad t_{1} \eta=\xi \quad t_{2} \xi=\eta \quad t_{2} \eta=\xi \quad\left\|t_{1}\right\|=\left\|t_{2}\right\|=1 .
$$

Then by Kadison transitivity, there are $a, b \in \mathfrak{A}$ such that

$$
\left.a\right|_{\mathcal{K}}=\left.t_{1}\right|_{\mathcal{K}},\left.\quad b\right|_{\mathcal{K}}=\left.t_{2}\right|_{\mathcal{K}} \quad\|a\|,\|b\| \leq 2
$$

Then since

$$
[a, b] \xi=[a b-b a] \xi=\left[t_{1} t_{2}-t_{2} t_{1}\right] \xi=t_{1} t_{2} \xi-t_{2} t_{1} \xi=t_{1} \eta+t_{2} \eta=2 \xi
$$

We have that $\|[a, b]\| \geq 2$.

The following result is known:

Theorem 8.4.2. Let $\mathcal{U}$ be an ultrafilter on $I$, and for all $i \in I$ suppose $\mathfrak{A}_{i}$ is a $\mathrm{C}^{*}$ algebra. Then $\prod_{\mathcal{U}} \mathfrak{A}_{i}$ is commutative if and only if $C=\left\{i \mid \mathfrak{A}_{i}\right.$ is commutative $\} \in \mathcal{U}$.

Proof. $(\Leftarrow)$ : This is clear.

$(\Rightarrow)$ : Suppose $N=\left\{i \mid \mathfrak{A}_{i}\right.$ is not commutative $\} \in \mathcal{U}$. Then for $i \in N$ we can pick $a_{i}, b_{i} \in \mathfrak{A}_{n}$ so that $\left\|a_{i}\right\|,\left\|b_{i}\right\| \leq 2$, and $\left\|\left[a_{i}, b_{i}\right]\right\| \geq 2$. Then consider $\left(a_{i}\right)_{\mathcal{U}}$ and $\left(b_{i}\right)_{\mathcal{U}}$,

$$
\left\|\left[\left(a_{n}\right)_{\mathcal{U}},\left(b_{n}\right)_{\mathcal{U}}\right]\right\|=\lim _{\mathcal{U}}\left\|\left[a_{n}, b_{n}\right]\right\| \geq 2
$$

Thus $\prod_{\mathcal{U}} \mathfrak{A}_{i}$ is not commutative as claimed. 
Comment: We know the axiom for a $\mathrm{C}^{*}$-algebra $\mathfrak{A}$ being commutative:

$$
\varphi_{c}=\sup _{\|x\|,\|y\| \leq 1}\|[x, y]\|=0
$$

We have shown an axiom for a $\mathrm{C}^{*}$-algebra $\mathfrak{A}$ being non-commutative:

$$
\varphi_{c}=\sup _{\|x\|,\|y\| \leq 1}\|[x, y]\|=2
$$

In other words, if every pair of elements in the unit ball of a $\mathrm{C}^{*}$-algebra almost commute, then the $\mathrm{C}^{*}$-algebra is commutative. Thus the commutative $\mathrm{C}^{*}$-algebras are an axiomatizable class, and the non-commutative $\mathrm{C}^{*}$-algebras are an axiomatizable class.

When we consider ultrapowers instead of ultraproducts, Theorems 8.3.2 and 8.4.2 are easier, and hold in the more general Banach algebra case. This was observed in Proposition 2.1 in [Daw09] and in section 1 of [Daw08].

\subsection{Finite $\mathrm{C}^{*}$-Algebras are Axiomatizable}

Recall the following definitions:

Definition 8.5.1. A unital $\mathrm{C}^{*}$-algebra is called directly finite or Dedekind finite if every left invertible element is right invertible, i.e. if $a b=I$ then $b a=I$.

Definition 8.5.2. A unital $\mathrm{C}^{*}$-algebra $\mathfrak{A}$ is Murray-von Neumann finite if for all $x$ with $x^{*} x=I$ we also have $x x^{*}=I$. In other words, isometries are unitaries. 
Recall that a projection which is not Murray-von Neumann equivalent to any of its subprojections is called finite. Conversely a projection which is equivalent to one of its proper subprojections is called infinite.

Proposition 8.5.3 (e.g. 5.1.2 in [RLL00]). For a unital $\mathrm{C}^{*}$-algebra $\mathfrak{A}$, the following are equivalent:

1. The identity of $\mathfrak{A}$ is a finite projection.

2. $\mathfrak{A}$ is Murray-von Neumann finite, i.e. all isometries are unitaries.

3. Every projection in $\mathfrak{A}$ is finite.

4. $\mathfrak{A}$ is Dedekind finite, i.e. every left-invertible element in $\mathfrak{A}$ is invertible.

5. Every right-invertible element in $\mathfrak{A}$ is invertible.

We will abusively refer to Dedekind or Murray-von Neumann finite $\mathrm{C}^{*}$-algebras as "finite", not to be confused with finite-dimensional.

Recall the polar decomposition theorem:

Theorem 8.5.4 (I.8.1 [Dav96]). Every operator $t \in \mathcal{B}(\mathcal{H})$ has a unique polar decomposition $t=u a$ where $a=|t|=\left(t^{*} t\right)^{1 / 2} \in C^{*}(t)$ and $u \in W^{*}(t)$, the von Neumann algebra generated by $t$, is a partial isometry. If $t$ is invertible, then $u \in C^{*}(t)$ is a unitary.

Proposition 8.5.5. An ultraproduct of finite $\mathrm{C}^{*}$-algebras is finite. 
Proof. Suppose $\left(x_{i}\right)_{\mathcal{U}}^{*}\left(x_{i}\right)_{\mathcal{U}}=\left(x_{i}^{*} x_{i}\right)_{\mathcal{U}}=\left(I_{i}\right)_{\mathcal{U}}$, so $\lim _{\mathcal{U}}\left\|x_{i}^{*} x_{i}-I_{i}\right\|=0$. Let $S=$ $\left\{i \mid\left\|x_{i}^{*} x_{i}-I_{i}\right\|<1\right\} \in \mathcal{U}$. For $i \in S, x_{i}^{*} x_{i}$ is invertible, so $x_{i}$ is left-invertible, and since $\mathfrak{A}_{i}$ is finite, $x_{i}$ is invertible. Let $x_{i}=u_{i}\left|x_{i}\right|$ be the polar decomposition of $x_{i}$ where $u_{i}$ is a unitary in $\mathfrak{A}_{i}$. Then

$$
0=\lim _{\mathcal{U}}\left\|x_{i}^{*} x_{i}-I_{i}\right\|=\lim _{\mathcal{U}}\|\mathrm{id}-1\|_{\infty, \sigma\left(x_{i}^{*} x_{i}\right)}
$$

We have that

$$
0=\lim _{\mathcal{U}}\|\sqrt{\mathrm{id}}-1\|_{\infty, \sigma\left(x_{i}^{*} x_{i}\right)}=\lim _{\mathcal{U}} \|\left|x_{i}\right|-I_{i}||
$$

Thus

$$
\left\|\left(x_{i}\right)_{\mathcal{U}}-\left(u_{i}\right)_{\mathcal{U}}\right\|=\lim _{\mathcal{U}}|| u_{i}\left|x_{i}\right|-u_{i}|| \leq \lim _{\mathcal{U}}|| u_{i}|| \|\left|x_{i}\right|-I_{i}||=0
$$

In particular, $\left(x_{i}\right)_{\mathcal{U}}=\left(u_{i}\right)_{\mathcal{U}}$. Finally, $\left(x_{i}\right)_{\mathcal{U}}\left(x_{i}\right)^{*}=\left(u_{i} u_{i}^{*}\right)_{\mathcal{U}}=\left(I_{i}\right)_{\mathcal{U}}$. Thus $\prod_{\mathcal{U}} \mathfrak{A}_{i}$ is finite as claimed.

Conversely, we have

Proposition 8.5.6. Let $\mathcal{U}$ be an ultrafilter on $I$, and for all $i \in I$ suppose $\mathfrak{A}_{i}$ is a $\mathrm{C}^{*}$-algebra. If $\prod_{\mathcal{U}} \mathfrak{A}_{i}$ is finite, then $\left\{i \mid \mathfrak{A}_{i}\right.$ is finite $\} \in \mathcal{U}$.

Proof. Suppose there is a set $S \in \mathcal{U}$ so for all $i \in S, \mathfrak{A}_{i}$ is not finite. Then for $i \in S$ there is an $x_{i} \in \mathfrak{A}_{i}$ with $x_{i}^{*} x_{i}=I$ and $x_{i} x_{i}^{*} \neq I$. In fact, we must have that $\left\|x_{i} x_{i}^{*}-I\right\| \geq 1$, since otherwise $x_{i} x_{i}^{*}$ is invertible, which means that $x_{i}$ is both left and right invertible, so $x_{i}$ is invertible, and $x_{i}^{*}=x_{i}^{-1}$ by the uniqueness of inverses. Thus $\left(x_{i}\right)_{\mathcal{U}} \in \prod_{\mathcal{U}} \mathfrak{A}_{i}$ satisfies $\left(x_{i}\right)_{\mathcal{U}}^{*}\left(x_{i}\right)_{\mathcal{U}}=\left(x_{i}^{*} x_{i}\right)_{\mathcal{U}}=\left(I_{i}\right)_{\mathcal{U}}$ but $\left\|\left(x_{i}\right)_{\mathcal{U}}\left(x_{i}\right)_{\mathcal{U}}^{*}-\left(I_{i}\right)_{\mathcal{U}}\right\|=$ $\lim _{\mathcal{U}}\left\|x_{i} x_{i}^{*}-I\right\| \geq 1$, so $\left(x_{i}\right)_{\mathcal{U}}\left(x_{i}\right)_{\mathcal{U}}^{*} \neq\left(I_{i}\right)_{\mathcal{U}}$. Thus $\prod_{\mathcal{U}} \mathfrak{A}_{i}$ is not finite. 
Thus we have shown that the collection of finite $\mathrm{C}^{*}$-algebras and the collection of non-finite $\mathrm{C}^{*}$-algebras are both axiomatizable classes.

In fact, there are explicit axioms for finite and stably finite $\mathrm{C}^{*}$-algebras, see 3.6(d) in $\left[\mathrm{FHL}^{+} 16\right]$.

\subsection{Stable Rank One is Axiomatizable}

The notion of stable rank was introduced by Rieffel in [Rie83]:

Definition 8.6.1. $\mathfrak{A}$ is stable rank $n$ if $n$ is the least integer such that the set of $n$-tuples over $\mathfrak{A}$ which generate $\mathfrak{A}$ as a left ideal are dense in $\mathfrak{A}^{n}$. If no such $n$ exists, we say $\mathfrak{A}$ is stable rank infinity.

Theorem 8.6.2 (3.3 in [Rie83]). A unital $\mathrm{C}^{*}$-algebra $\mathfrak{A}$ is stable rank one if and only if the invertible elements are dense in $\mathfrak{A}$.

Proposition 8.6.3. An ultraproduct of stable rank one algebras is stable rank one.

Proof. Suppose $\mathfrak{A}_{i}$ is stable rank 1 for all $i$. Let $\varepsilon>0$ be given. Then for any $\left(x_{i}\right)_{\mathcal{U}} \in \prod_{\mathcal{U}} \mathfrak{A}_{i}$, we can find $y_{i} \in \operatorname{GL}\left(\mathfrak{A}_{i}\right)$ so $\left\|x_{i}-y_{i}\right\|<\varepsilon / 2$. Then since $y_{i}$ is invertible, by the polar decomposition theorem we have that $y_{i}=u_{i}\left|y_{i}\right|$ where $u_{i}$ is a unitary, $\left|y_{i}\right|=\left(y_{i}^{*} y_{i}\right)^{1 / 2}$, and both $u_{i},\left|y_{i}\right| \in \mathfrak{A}_{i}$. Then let $z_{i}=u_{i}\left(\left|y_{i}\right|+\frac{\varepsilon}{2} I_{i}\right) \in \mathfrak{A}_{i}$, then $z_{i}$ is invertible, and

$$
\left\|x_{i}-z_{i}\right\| \leq\left\|x_{i}-y_{i}\right\|+\left\|u_{i} \frac{\varepsilon}{2} I_{i}\right\| \leq \varepsilon .
$$


Now we have that

$$
\left\|z_{i}^{-1}\right\|=\left\|\left(\left|y_{i}\right|+\frac{\varepsilon}{2} I_{i}\right)^{-1} u_{i}^{-1}\right\|=\left\|\left(\left|y_{i}\right|+\frac{\varepsilon}{2} I_{i}\right)^{-1}\right\| \leq \frac{2}{\varepsilon}
$$

via the functional calculus. Thus $\left(z_{i}\right)_{\mathcal{U}} \in \prod_{\mathcal{U}} \mathfrak{A}_{i}$ is invertible, and $\left\|\left(x_{i}\right)_{\mathcal{U}}-\left(z_{i}\right)_{\mathcal{U}}\right\|=$ $\lim _{\mathcal{U}}\left\|x_{i}-z_{i}\right\| \leq \varepsilon$. Thus the invertible elements of $\prod_{\mathcal{U}} \mathfrak{A}_{i}$ are dense in $\prod_{\mathcal{U}} \mathfrak{A}_{i}$, so $\prod_{\mathcal{U}} \mathfrak{A}_{i}$ has stable rank one.

Proposition 8.6.4. A $C^{*}$-algebra $\mathfrak{A}$ is stable rank one if and only if $\mathfrak{A}^{\mathcal{U}}$ is stable rank one.

Proof. We have already shown that if $\mathfrak{A}$ is stable rank one, then $\mathfrak{A}^{\mathcal{U}}$ is stable rank one. Conversely, if $\mathfrak{A}^{\mathcal{U}}$ is stable rank one, then given $x \in \mathfrak{A}$ and $\varepsilon>0$, then consider $(x)_{\mathcal{U}} \in \mathfrak{A}^{\mathcal{U}}$. Since $\mathfrak{A}^{\mathcal{U}}$ is stable rank one, there is an invertible $\left(y_{i}\right)_{\mathcal{U}} \in \mathfrak{A}^{\mathcal{U}}$ so that $\left\|(x)_{\mathcal{U}}-\left(y_{i}\right)_{\mathcal{U}}\right\|<\varepsilon / 2$. Then $\left\{i: y_{i} \in \operatorname{GL}(\mathfrak{A})\right\} \cap\left\{i:\left\|x_{i}-y_{i}\right\|<\varepsilon\right\} \in \mathcal{U}$ so there is an $i$ so $y_{i}$ is invertible and $\left\|x_{i}-y_{i}\right\|<\varepsilon$, so $\mathfrak{A}$ is stable rank one.

The following result is due to Rørdam:

Theorem 8.6.5 (2.6 in $[\mathrm{R} \varnothing \mathrm{r} 88])$. If $\mathfrak{A}$ is a $\mathrm{C}^{*}$-algebra with stable rank not equal to one, then there is an $x \in \mathfrak{A}$ with $\|x\|=1=\operatorname{dist}(x, \operatorname{GL}(\mathfrak{A}))$.

Corollary 8.6.6. Let $\mathcal{U}$ be an ultrafilter on $I$, and suppose for all $i \in I$ that $\mathfrak{A}_{i}$ is a $C^{*}$ algebra. Then $\prod_{\mathcal{U}} \mathfrak{A}_{i}$ is stable rank one if and only if $\left\{i \mid \mathfrak{A}_{i}\right.$ is stable rank one $\} \in \mathcal{U}$.

Thus the stable rank one $\mathrm{C}^{*}$-algebras are an axiomatizable class, and the nonstable rank one $\mathrm{C}^{*}$-algebras are an axiomatizable class. In fact, there are explicit 
axioms for being stable rank one, being stable rank less than $n$ for all $n \in \mathbb{N}$. See Lemmas 3.7.2-3.7.4 in $\left[\mathrm{FHL}^{+} 16\right]$.

\subsection{Unitary Polar Decompositions}

Since unitary polar decompositions were useful in the previous two sections, we explore them a little further.

Proposition 8.7.1. If for all $i$, every element of $\mathfrak{A}_{i}$ has a unitary polar decomposition, then every element in $\prod_{\mathcal{U}} \mathfrak{A}_{i}$ has a unitary polar decomposition.

Proof. Since $M_{n}$ is a von Neumann algebra, every element has a unitary polar decomposition. Then if $\left(a_{i}\right)_{\mathcal{U}} \in \prod_{\mathcal{U}} M_{n_{i}}$, we have that $a_{i}=u_{i}\left|a_{i}\right|$ for some unitary $u_{i} \in M_{n_{i}}$. Thus

$$
\left(a_{i}\right)_{\mathcal{U}}=\left(u_{i}\left|a_{i}\right|\right)_{\mathcal{U}}=\left(u_{i}\right)_{\mathcal{U}}\left|\left(a_{i}\right)_{\mathcal{U}}\right|
$$

since $\sqrt{\cdot}$ is a uniform limit of polynomials on $\left[0, \sup _{i}\left\|a_{i}\right\|\right]$.

Proposition 8.7.2. If every element of $\mathfrak{A}^{\mathcal{U}}$ has a unitary polar decomposition, then every element of $\mathfrak{A}$ is a norm-limit of unitary polar decompositions. That is, there is a sequence of unitaries $u_{n} \in \mathfrak{A}$ so $u_{n}|a|$ converges to $a$.

Proof. Let $a \in \mathfrak{A}$ be given, then $(a)_{\mathcal{U}}=u\left|(a)_{\mathcal{U}}\right|=\left(u_{i}|a|\right)_{\mathcal{U}}$ for some unitaries $u_{i} \in \mathfrak{A}$. Thus $\lim _{\mathcal{U}}\left\|a-u_{i}|a|\right\|=0$, and we have that $a$ is a norm-limit of unitary polar decompositions as claimed. 
Corollary 8.7.3. In pseudocompact $\mathrm{C}^{*}$-algebras, every element is a norm-limit of unitary polar decompositions.

Question 8.7.4. If $a$ is a norm-limit of unitary polar decompositions, does $a$ have a unitary polar decomposition?

\subsection{Real Rank Zero is Axiomatizable}

Recall the following definition, due to Brown and Pedersen in [BP91]:

Definition 8.8.1. A $\mathrm{C}^{*}$-algebra $\mathfrak{A}$ is said to have real rank zero if the self-adjoint elements with finite spectra are dense in the self-adjoint elements of $\mathfrak{A}$.

This is equivalent to the invertible self-adjoint elements being dense in the selfadjoint elements. It implies that the span of the projections is dense (since the self-adjoint elements span the algebra, and each self-adjoint element is close to a self-adjoint element with finite spectrum, which is a linear combination of orthogonal projections by the spectral theorem). Like stable rank, there is a notion of being real rank $n$ for all $n \in \mathbb{N}$.

Theorem 8.8.2. If $\mathfrak{A}^{\mathcal{U}}$ is real rank zero, then $\mathfrak{A}$ is real rank zero.

Proof. Suppose $\mathfrak{A}^{\mathcal{U}}$ has real rank zero. Let $x \in \mathfrak{A}_{s a}$ and $\varepsilon>0$ be given. Then $(x)_{\mathcal{U}} \in\left(\mathfrak{A}^{\mathcal{U}}\right)_{s a}$, so there is a self-adjoint element $\left(y_{i}\right)_{\mathcal{U}} \in\left(\mathfrak{A}^{\mathcal{U}}\right)_{s a}$ with finite spectrum, and $\left\|\left(x_{i}\right)_{\mathcal{U}}-\left(y_{i}\right)_{\mathcal{U}}\right\|<\frac{\varepsilon}{3}$. Without loss of generality $y_{i} \in \mathfrak{A}_{s a}$ for all $i$. Then

$$
S=\left\{i:\left\|y_{i}-x_{i}\right\|<\frac{2 \varepsilon}{3}\right\} \in \mathcal{U}
$$


Let $\sigma\left(\left(y_{i}\right)_{\mathcal{U}}\right)=\left\{\lambda_{1}, \ldots, \lambda_{N}\right\}$. Pick disjoint open balls $U_{1}, \ldots, U_{N}$ so $\lambda_{j} \in U_{j}$ and the diameter of each $U_{j}$ is less than $\frac{\varepsilon}{3}$. Then since

$$
\left\{\lambda_{1}, \ldots, \lambda_{N}\right\}=\sigma\left(\left(y_{i}\right)_{\mathcal{U}}\right)=\lim _{\mathcal{U}} \sigma\left(y_{n}\right)
$$

we have

$$
T=\left\{i \mid \sigma\left(y_{i}\right) \subseteq \bigcup_{j=1}^{N} U_{j}\right\} \in \mathcal{U} .
$$

Otherwise for all $i \in[I \backslash S] \in \mathcal{U}$ there would be a $\mu_{i} \in \sigma\left(y_{i}\right)$ with $\left|\mu_{i}-\lambda_{j}\right| \geq \operatorname{diam}\left(U_{j}\right)$, then $\lim _{\mathcal{U}} \mu_{i} \in \lim _{\mathcal{U}} \sigma\left(y_{i}\right)=\sigma\left(\left(y_{i}\right)_{\mathcal{U}}\right)$ but $\lim _{\mathcal{U}} \mu_{i} \neq \lambda_{1}, \ldots, \lambda_{N}$, a contradiction.

Then for $i \in S \cap T \in \mathcal{U}$, we can consider the piecewise constant function

$$
f=\sum_{j=1}^{N} \lambda_{j} 1_{U_{j}} \in \mathcal{C}\left(\sigma\left(y_{i}\right)\right) .
$$

Thus we can let $z_{i}=f\left(y_{i}\right)$. Then

$$
\sigma\left(z_{i}\right)=f\left(\sigma\left(y_{i}\right)\right) \subseteq\left\{\lambda_{1}, \ldots, \lambda_{N}\right\}
$$

So $z_{i}$ is self-adjoint and has finite spectrum, and

$$
\left\|x_{i}-z_{i}\right\| \leq\left\|x_{i}-y_{i}\right\|+\left\|y_{i}-z_{i}\right\|<\frac{2 \varepsilon}{3}+\|\mathrm{id}-f\|_{\infty, \sigma\left(y_{i}\right)}<\varepsilon
$$

Thus $\mathfrak{A}$ has real rank zero.

Theorem 8.8.3. An ultraproduct of real rank zero $\mathrm{C}^{*}$-algebras has real rank zero.

Proof. Suppose for all $i, \mathfrak{A}_{i}$ has real rank zero. Let $\left(x_{i}\right)_{\mathcal{U}} \in\left(\prod_{\mathcal{U}} \mathfrak{A}_{i}\right)_{s a}$ and $\varepsilon>0$ be given. Without loss of generality we can assume $x_{i} \in\left(\mathfrak{A}_{i}\right)_{s a}$ for all $i$. Then for all $i$ we 
can find $y_{i} \in\left(\mathfrak{A}_{i}\right)_{s a}$ so $\left\|x_{i}-y_{i}\right\|<\frac{\varepsilon}{2}$ and $\sigma\left(y_{i}\right)$ is finite. Let $M=\sup _{n}\left\|x_{i}\right\|+\frac{\varepsilon}{2}<\infty$.

Then $\sigma\left(y_{i}\right) \subseteq[-M, M]$. Let $N=\left\lceil\frac{4 M}{\varepsilon}\right\rceil$. Since $\sigma\left(y_{i}\right)$ is finite,

$$
f=\sum_{j=0}^{N}\left(-M+j \frac{\varepsilon}{2}\right) 1_{\left[-M+j \frac{\varepsilon}{2},-M+(j+1) \frac{\varepsilon}{2}\right)} \in \mathcal{C}\left(\sigma\left(y_{i}\right)\right) .
$$

Thus we can consider $z_{i}=f\left(y_{i}\right)$. Note that

$$
\sigma\left(z_{i}\right)=f\left(\sigma\left(y_{i}\right)\right) \subseteq\left\{-M,-M+\frac{\varepsilon}{2},-M+\frac{2 \varepsilon}{2}, \ldots,-M+\frac{N \varepsilon}{2}\right\}
$$

Then $z_{i}$ is self-adjoint and

$$
\left\|x_{i}-z_{i}\right\| \leq\left\|x_{i}-y_{i}\right\|+\left\|y_{i}-z_{i}\right\|<\frac{\varepsilon}{2}+\|\mathrm{id}-f\|_{\infty, \sigma\left(y_{i}\right)}<\varepsilon
$$

Thus we can consider $\left(z_{i}\right)_{\mathcal{U}} \in\left(\prod_{\mathcal{U}} \mathfrak{A}_{i}\right)_{s a}$, which satisfies

$$
\left\|\left(x_{i}\right)_{\mathcal{U}}-\left(z_{i}\right)_{\mathcal{U}}\right\|=\lim _{\mathcal{U}}\left\|x_{i}-z_{i}\right\| \leq \varepsilon
$$

and

$$
\sigma\left(\left(z_{i}\right)\right)=\lim _{\mathcal{U}} \sigma\left(z_{i}\right) \subseteq\left\{-M,-M+\frac{\varepsilon}{2},-M+\frac{2 \varepsilon}{2}, \ldots,-M+\frac{N \varepsilon}{2}\right\}
$$

Thus $\prod_{\mathcal{U}} \mathfrak{A}_{i}$ is real rank zero as claimed.

Thus the real rank zero $\mathrm{C}^{*}$-algebras are an axiomatizable class.

In fact, there are explicit axioms for being real rank zero, real rank greater than zero, and real rank $\leq n$ for all $n \in \mathbb{N}$. See Example 2.4.2, 3.6(b), and Proposition 3.7.5 in $\left[\mathrm{FHL}^{+} 16\right]$. We will call the axiom for being real rank zero $\varphi_{\operatorname{rr} 0}$. 


\subsection{Tracial $\mathrm{C}^{*}$-Algebras are Axiomatizable}

Here, a trace on a unital $\mathrm{C}^{*}$-algebra $\mathfrak{A}$ is a tracial state: a positive, bounded linear functional $\tau: \mathfrak{A} \rightarrow \mathbb{C}$ so $\tau\left(a^{*} a\right) \geq 0, \tau(I)=1$, and $\tau(a b)=\tau(b a)$ for all $a, b \in \mathfrak{A}$. We say a unital $\mathrm{C}^{*}$-algebra with a tracial state is a tracial $\mathrm{C}^{*}$-algebra. For instance, on $M_{n}$ we take the normalized trace of a matrix $A=\left[a_{i, j}\right] \in M_{n}$ by taking a basis $\xi_{1}, \ldots, \xi_{n}$, then

$$
\tau(A)=\frac{1}{n} \sum_{i=1}^{n} a_{i, i}=\frac{1}{n} \sum_{i=1}^{n}\left\langle A \xi_{i} \mid \xi_{i}\right\rangle .
$$

Proposition 8.9.1 (e.g. 5.4 in [GH01]). An ultraproduct of tracial $\mathrm{C}^{*}$-algebras is tracial.

Proof. If $\tau_{i}$ is a tracial state on $\mathfrak{A}_{i}$, then $\tau_{\mathcal{U}}\left(\left(a_{i}\right)\right)=\lim _{\mathcal{U}} \tau_{i}\left(a_{i}\right)$ defines a tracial state on $\prod_{\mathcal{U}} \mathfrak{A}_{i}$.

We have seen in Example 7.3.3 that even if the traces on the components are faithful, the limit trace on the ultraproduct need not be faithful. Traces on ultraproducts are studied in [BF15].

Proposition 8.9.2. $\mathfrak{A}$ has a tracial state if and only if $\mathfrak{A}^{\mathcal{U}}$ has a tracial state.

Proof. We have already seen that if $\mathfrak{A}$ has a tracial state then $\mathfrak{A}^{\mathcal{U}}$. Conversely, if $\tau_{\mathcal{U}}$ is a trace on $\mathfrak{A}^{\mathcal{U}}$ we get a trace on $\mathfrak{A}$ by restricting $\tau_{\mathcal{U}}$ to the image of the diagonal embedding $a \mapsto(a)_{\mathcal{U}}$.

Thus the tracial $\mathrm{C}^{*}$-algebras are an axiomatizable class. In fact, an explicit axiomatization is given in $3.4(\mathrm{a})$ in $\left[\mathrm{FHL}^{+} 16\right]$. 


\section{Chapter 9}

\section{Pseudocompact and}

\section{Pseudomatrical $\mathrm{C}^{*}$-Algebras}

\subsection{Definition of Pseudocompactness}

The term pseudofinite harkens back to [Ax68]. An infinite field is pseudofinite if it satisfies every sentence which is satisfied in every finite field. Most of the research on pseudofiniteness has been restricted to fields and groups. For some results on pseudofinite groups see e.g. [Wil95] and [OHP13], and for some general results on pseudofinite model theory see [Vää03]. The analogous property in continuous logic is called pseudocompactness and was introduced in [GL15].

Proposition 9.1.1 (2.4 in [GL15]). The following are equivalent for a $\mathrm{C}^{*}$-algebra $\mathfrak{A}$ :

1. Let $\varphi$ be a sentence in the language of $\mathrm{C}^{*}$-algebras. If $\varphi^{\mathcal{F}}=0$ for all finite- 
dimensional $\mathrm{C}^{*}$-algebras $\mathcal{F}$ then $\varphi^{\mathfrak{A}}=0$.

2. Let $\psi$ be a sentence in the language of $\mathrm{C}^{*}$-algebras. If $\psi^{\mathfrak{A}}=0$ then for all $\varepsilon>0$ there is a finite-dimensional $\mathrm{C}^{*}$-algebra $\mathcal{F}$ so that $\left|\psi^{\mathcal{F}}\right|<\varepsilon$.

3. $\mathfrak{A}$ is elementarily equivalent to an ultraproduct of finite-dimensional $\mathrm{C}^{*}$-algebras.

Definition 9.1.2. We say that $\mathfrak{A}$ is a pseudocompact $\mathrm{C}^{*}$-algebras if it satisfies any of the above conditions. If one replaces every occurrence of "finite-dimensional" with "matrix algebra", then we call $\mathfrak{A}$ a pseudomatricial $\mathrm{C}^{*}$-algebra. We do not require $\mathfrak{A}$ to be infinite-dimensional, but perhaps it is natural to do so.

Again, pseudocompact $\mathrm{C}^{*}$-algebras can be thought of as the limit points of the finite-dimensional $\mathrm{C}^{*}$-algebras with respect to logic. The pseudocompact $\mathrm{C}^{*}$-algebras and the pseudomatricial $\mathrm{C}^{*}$-algebras are the smallest axiomatizable classes containing the finite-dimensional $\mathrm{C}^{*}$-algebras and matrix algebras respectively, i.e. the smallest classes closed under ultraproducts and ultraroots.

We are specifically interested in the separable, infinite-dimensional pseudomatricial $\mathrm{C}^{*}$-algebras, which can be obtained by taking an ultraproduct of the form $\prod_{\mathcal{U}} M_{k_{n}}$ where $\mathcal{U}$ is a free ultrafilter on the natural numbers and the $k_{n}$ are unbounded, then using the downwards Löwenheim-Skolem theorem to take a separable elementary submodel.

Because of the way sentences are constructed in continuous logic, we are particularly interested in the evaluations of polynomials on matrices, which has been studied 
in [KBMR12] and [KBMR16]. Likewise, we are interested in when the norm of a polynomial in several matrices is small implies that there is a nearby zero of that polynomial. These were studied in [TT94].

See Section 5 in [FHS14b] for the definition and some results about pseudocompact tracial von Neumann algebras. In particular, it was shown that pseudocompact tracial von Neumann algebras do not have property $\Gamma$, so the hyperfinite $\mathrm{II}_{1}$ factor is not a pseudocompact von Neumann algebra.

\subsection{Stability}

We study some operations which preserve pseudocompactness. Notice that the first definition of pseudocompactness and Łos' theorem imply that an ultraproduct of pseudocompact $\mathrm{C}^{*}$-algebras is pseudocompact. We have shown that direct sums and tensoring with matrix algebras commute with ultraproducts $(2.7 .1,2.7 .2)$, so by appealing to the Keisler-Shelah theorem we see the pseudocompact $\mathrm{C}^{*}$-algebras are closed under these operations. Similarly the pseudomatricial $\mathrm{C}^{*}$-algebras are stable under tensoring with matrix algebras. The next result implies that corners of pseudocompact $\mathrm{C}^{*}$-algebras are pseudocompact.

Proposition 9.2.1. If $p$ is a projection in a pseudocompact $\mathrm{C}^{*}$-algebra $\mathfrak{A}$, then $p \mathfrak{A} p$ is pseudocompact.

Proof. Suppose $\mathfrak{A}$ is pseudocompact. Then by the Keisler-Shelah theorem there 
are finite-dimensional $\mathrm{C}^{*}$-algebras $F_{i}$ and ultrafilters $\mathcal{U}, \mathcal{V}$ and a $*$-isomorphism $\phi$ : $(\mathfrak{A})^{\mathcal{V}} \rightarrow\left(\prod_{i \rightarrow \mathcal{U}} F_{i}\right)^{\mathcal{V}}$. Let $p$ be a projection in $\mathfrak{A}$, then $\phi\left((p)_{j \rightarrow \mathcal{V}}\right)$ is a projection in $\left(\prod_{i \rightarrow \mathcal{U}} F_{i}\right)^{\mathcal{V}}$, so it has a representative sequence of projections. Thus there are projections $p_{i}^{(j)} \in F_{i}$ so that $\phi\left((p)_{\mathcal{V}}\right)=\left(\left(p_{i}^{(j)}\right)_{i \rightarrow \mathcal{U}}\right)_{j \rightarrow \mathcal{V}}$. We claim that $(p \mathfrak{A} p)^{\mathcal{V}} \cong$

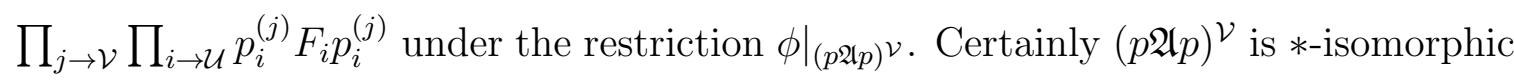
to its image. Suppose $\left(p a^{(j)} p\right)_{j \rightarrow \mathcal{V}} \in(p \mathfrak{A} p)^{\mathcal{V}}$. Let

$$
\phi\left(\left(p a^{(j) p}\right)\right)=\left(\left(b_{i}^{(j)}\right)_{i \rightarrow \mathcal{U}}\right)_{j \rightarrow \mathcal{V}} \in\left(\prod_{\mathcal{U}} F_{i}\right)^{\mathcal{V}} .
$$

Then the image of $\left(p a^{(j)} p\right)_{j \rightarrow \mathcal{V}}$ under $\phi$ is

$$
\begin{aligned}
\phi\left(\left(p a^{(j)} p\right)_{j \rightarrow \mathcal{V}}\right) & =\phi\left((p)_{j \rightarrow \mathcal{V}}\right) \phi\left(\left(p a^{(j)} p\right)_{j \rightarrow \mathcal{V}}\right) \phi\left((p)_{j \rightarrow \mathcal{V}}\right) \\
& =\left(\left(p_{i}^{(j)}\right)_{i \rightarrow \mathcal{U}}\right)_{j \rightarrow \mathcal{V}}\left(\left(b_{i}^{(j)}\right)_{i \rightarrow \mathcal{U}}\right)_{j \rightarrow \mathcal{V}}\left(\left(p_{i}^{(j)}\right)_{i \rightarrow \mathcal{U}}\right)_{j \rightarrow \mathcal{V}} \\
& =\left(\left(p_{i}^{(j)} b_{i}^{(j)} p_{i}^{(j)}\right)_{i \rightarrow \mathcal{U}}\right)_{j \rightarrow j} \in \prod_{j \rightarrow \mathcal{V}} \prod_{i \rightarrow \mathcal{U}} p_{i}^{(j)} F_{i} p_{i}^{(j)} .
\end{aligned}
$$

In fact this shows $\left.\phi\right|_{(p \mathfrak{A} p)^{\mathcal{V}}}$ maps onto $\prod_{j \rightarrow \mathcal{V}} \prod_{i \rightarrow \mathcal{U}} p_{i}^{(j)} F_{i} p_{i}^{(j)}$ since $\phi$ maps $\mathfrak{A}^{\mathcal{V}}$ onto $\left(\prod_{\mathcal{U}} F_{i}\right)^{\mathcal{V}}$. An element of $\prod_{j \rightarrow \mathcal{V}} \prod_{i \rightarrow \mathcal{U}} p_{i}^{(j)} F_{i} p_{i}^{(j)}$ is of the form $\left(\left(p_{i}^{(j)} b_{i}^{(j)} p_{i}^{(j)}\right)_{i \rightarrow \mathcal{U}}\right)_{j \rightarrow \mathcal{V}}$ with $b_{i}^{j} \in F_{i}$. Then $\left(p_{i}^{(j)} b_{i}^{(j)} p_{i}^{(j)}\right)_{j \rightarrow \mathcal{V}}=\phi\left(\left(a^{(j)}\right)_{j \rightarrow \mathcal{V}}\right)$ for some $\left(a^{(j)}\right)_{j \rightarrow \mathcal{V}} \in \mathfrak{A}^{\mathcal{V}}$. Then $\phi$ maps $\left(p a^{(j)} p\right)_{j \rightarrow \mathcal{V}} \in(p \mathfrak{A} p)^{\mathcal{V}}$ onto the desired element. Thus we have

$$
(p \mathfrak{A} p)^{\mathcal{V}} \cong \prod_{j \rightarrow \mathcal{V}} \prod_{i \rightarrow \mathcal{U}} p_{i}^{(j)} F_{i} p_{i}^{(j)}
$$

So $p \mathfrak{A} p$ is pseudocompact.

We will see in 11.4.7 that the center of a pseudocompact $\mathrm{C}^{*}$-algebra is pseudocompact, but subalgebras of pseudocompact $\mathrm{C}^{*}$-algebras do not need to be pseudo- 
compact.

Question 9.2.2. Is an ultraproduct of group $C^{*}$-algebras associated to finite groups the group $\mathrm{C}^{*}$-algebra of a pseudofinite group?

Is an ultraproduct of graph $\mathrm{C}^{*}$-algebras associated to finite graphs the graph $\mathrm{C}^{*}$ algebra of a pseudofinite graph? 


\section{Chapter 10}

\section{Commutative Pseudocompact}

\section{$\mathrm{C}^{*}$-Algebras}

We briefly recount the history and important results about commutative pseudocompact $\mathrm{C}^{*}$-algebras. We conclude with an axiomatization of the commutative pseudocompact $\mathrm{C}^{*}$-algebras and some examples.

Pseudocompact real Banach spaces and commutative Banach algebras were studied by Henson and Moore, using non-standard hulls and Henson's positive bounded formulae with approximate semantics, and using the unfortunate terminology hyperfinite and almost-finite. See for instance [Hen76] and [Moo81]. Some of their results were restated in terms of ultraproducts and for $\mathrm{C}^{*}$-algebras in Heinrich's survey paper [Hei80]. Most of the arguments required to adapt Henson's and Moore's classification result for $\mathrm{C}^{*}$-algebras are contained in a paper by Eagle and Vignati [EV14]. 


\subsection{Commutative $\mathrm{C}^{*}$-Algebras}

Recall that a commutative unital $\mathrm{C}^{*}$-algebra $\mathfrak{A}$ is $*$-isomorphic to $\mathcal{C}(K)$ where $K$ is a compact Hausdorff space, by considering elements of $\mathfrak{A}$ as continuous functions on the maximal ideal space of $\mathfrak{A}$. Conversely, every multiplicative linear functional on $\mathcal{C}(K)$ is a point evaluation (and every point evaluation is a multiplicative linear functional).

Proposition 1.1 in [BP91] states that if $K$ is compact Hausdorff then the real rank of $\mathcal{C}(K)$ is the dimension of $K$. A (locally) compact Hausdorff spaces is zerodimensional if and only if it is totally disconnected. Thus $\mathcal{C}(K)$ is real rank zero if and only if $K$ is totally disconnected.

Furthermore $\mathcal{C}(K)$ is separable if and only if $K$ is metrizable. $K$ is metrizable and totally disconnected if and only if $\mathcal{C}(K)$ is an $\mathrm{AF} \mathrm{C}^{*}$-algebra.

Note that the finite-dimensional commutative $\mathrm{C}^{*}$-algebras are exactly those $\mathcal{C}(K) \cong$ $\mathbb{C}^{d}$ where $K$ is finite of cardinality $d$ and discrete.

\subsection{Ultraproducts of Commutative $\mathrm{C}^{*}$-Algebras}

Ultraproducts of $\mathcal{C}(K)$ spaces are $\mathcal{C}(K)$ spaces:

Theorem 10.2.1 (3.1 in [Hen76], 3.3 in [Hei80]). Let $\mathcal{U}$ be an ultrafilter on $I$ and for all $i \in I$ suppose $K_{i}$ is a compact Hausdorff space. Then there is a compact Hausdorff space $K$ so that the ultraproduct $\prod_{\mathcal{U}} \mathcal{C}\left(K_{i}\right)$ is $*$-isomorphic to $\mathcal{C}(K)$.

This is clear, since $\prod_{\mathcal{U}} \mathcal{C}\left(K_{i}\right)$ is unital and commutative. 
In fact, if $\mathfrak{A}$ is a commutative pseudocompact $\mathrm{C}^{*}$-algebra, then $\mathfrak{A} \equiv \prod_{\mathcal{U}} F_{i}$ for some finite-dimensional $\mathrm{C}^{*}$-algebras $F_{i}$ of dimension $d_{i}$, but since $\mathfrak{A}$ is commutative, and non-commutativity is an axiomatizable property, we can assume all the $F_{i}$ are commutative, so $F_{i} \cong \mathbb{C}^{d_{i}}$. Likewise the right-hand side is unital, and this is an axiomatizable property, so $\mathfrak{A}$ is unital. Thus by Gelfand's representation theorem, $\mathfrak{A} \cong \mathcal{C}(K)$ for some compact Hausdorff $K$. We are interested in the topological properties of this space $K$.

When we have $\prod_{\mathcal{U}} \mathcal{C}\left(K_{i}\right) \cong \mathcal{C}(K)$, the structure of $K$ is closely related to the $K_{i}$. Consider the set-theoretic ultraproduct $\prod_{\mathcal{U}} K_{i}$. Then the family of subsets $\left(U_{i}\right)_{\mathcal{U}}$ where $U_{i}$ is an open subset of $K_{i}$, forms a basis of a topology on $\prod_{\mathcal{U}} K_{i}$. We will always use this natural topology on the ultraproduct. For a topological space $X$, $B(X)$ is the collection of clopen sets in $X$ (which is a Boolean algebra).

Theorem 10.2.2 (4.1 in [Hei80]). Let $\mathcal{U}$ be an ultrafilter on $I$ and for all $i \in I$ suppose $K_{i}$ is a compact Hausdorff space. Let $K$ be the compact Hausdorff space satisfying $\mathcal{C}(K) \cong \prod_{\mathcal{U}} \mathcal{C}\left(K_{i}\right)$. Then we have the following:

1. $\prod_{\mathcal{U}} K_{i}$ is canonically homeomorphic to a dense subset of $K$.

2. A subset $A \subseteq K$ belongs to the clopen subsets of $K$, i.e. $A \in B(K)$ if and only if it is the closure in $K$ of an element of $\prod_{\mathcal{U}} B\left(K_{i}\right)$.

3. If the spaces $K_{i}$ are totally disconnected, then so is $K$.

4. If the spaces $K_{i}$ have dense subsets of isolated points, then so does $K$. 
Not all topological properties of the $K_{i}$ pass to the space $K$ with $\mathcal{C}(K) \cong \prod_{\mathcal{U}} \mathcal{C}\left(K_{i}\right)$.

Example 10.2.3 (2.6, 2.7 in [HM74], $\S 8$ in [Sim82]). Recall a topological space is extremally disconnected if the closure of every open set is itself open. An extremely disconnected compact Hausdorff space is a Stonean space. For example, the StoneČech compactification of a discrete space is Stonean, in particular, $\beta(\mathbb{N})$ is a Stonean space.

Recall in the category of Banach spaces with contractive linear maps, a Banach space $I$ is injective if for all Banach spaces $A$ and $B$ with $A$ a subspace of $B$ and $\phi: A \rightarrow I$ a contractive linear map, there is an extension of $\phi$ to $\Phi: B \rightarrow I$ a contractive linear map. For example, $\mathbb{C}$ is injective by the Hahn-Banach theorem, and $\ell_{\infty}(\mathbb{N})$ is injective by using Hahn-Banach component-wise (and in general the direct sum of injective spaces is injective). Since every Banach space embeds isometrically into an injective space, $B \hookrightarrow \ell_{\infty}\left(B_{\leq 1}^{*}\right)$, there are sufficiently many injectives, and being injective is equivalent to being contractively complemented in any super Banach space, which is equivalent to being contractively complemented in one super Banach space. If $K$ is Stonean then $\mathcal{C}(K)$ is an injective Banach space, and these are all the injective Banach spaces.

If $\mathcal{U}$ is a non-principal ultrafilter on $\mathbb{N}$, then there is a natural isometric embedding

$$
\mathcal{C}(K)=\left(\ell_{\infty}(\mathbb{N})\right)^{\mathcal{U}} \hookrightarrow \ell_{\infty}\left(\mathbb{N}^{\mathcal{U}}\right)
$$

The map sends $f=\left(f_{i}\right)_{\mathcal{U}} \in\left(\ell_{\infty}(\mathbb{N})\right)^{\mathcal{U}}$ to $\Phi_{f} \in \ell_{\infty}\left(\mathbb{N}^{\mathcal{U}}\right)$, i.e. $\Phi_{f}: \mathbb{N}^{\mathcal{U}} \rightarrow \mathbb{C}$ is bounded 
map. $\Phi_{f}$ is defined by

$$
\Phi_{f}\left(\left(n_{i}\right) \mathcal{U}\right)=\lim _{\mathcal{U}} f_{i}\left(n_{i}\right)
$$

Note that $\ell_{\infty}(\mathbb{N})=\mathcal{C}(\beta(\mathbb{N}))$, where $\beta(\mathbb{N})$ is extremally disconnected. One can show via contradiction that the space $K$ with $\mathcal{C}(K) \cong\left(\ell_{\infty}(\mathbb{N})\right)^{\mathcal{U}}$ is not extremally disconnected. Were $K$ extremally disconnected, then $\mathcal{C}(K)$ would injective, so it would have to be contractively complemented in $\ell_{\infty}\left(\mathbb{N}^{\mathcal{U}}\right)$. This bounded projection of $\ell_{\infty}\left(\mathbb{N}^{\mathcal{U}}\right)$ onto $\left(\ell_{\infty}(\mathbb{N})\right)^{\mathcal{U}}$ would imply the existence of a bounded projection from $\ell_{\infty}(\mathbb{N})$ onto $c_{0}(\mathbb{N})$ which is known to be impossible. See the discussion following Theorem 4 in $\S 8$ in [Sim82]. Thus the ultraproduct of $\mathcal{C}(K)$ with $K$ extremally disconnected can be a $\mathcal{C}(K)$ space where $K$ is not extremally disconnected.

However, the topological properties of being totally disconnected and having a dense set of isolated points (see 10.3.7) turn out to be better behaved:

Proposition 10.2.4 (3.5 in [Hen76]). Let $X, Y$ be compact Hausdorff spaces and suppose $\mathcal{C}(X) \equiv \mathcal{C}(Y)$ as real Banach spaces (only equipped with addition and scalar multiplication). If $X$ is totally disconnected then $Y$ is also.

\subsection{Commutative Pseudocompact $\mathrm{C}^{*}$-Algebras}

Notice that if $\mathcal{C}(X) \equiv \mathcal{C}(Y)$ as $\mathrm{C}^{*}$-algebras they necessarily are elementarily equivalent as real Banach spaces as well: a Banach space statement is a $\mathrm{C}^{*}$-statement, and we can replace variables $x$ with $\left(x+x^{*}\right) / 2$ to force them to be real-valued. Thus if $\mathfrak{A}$ 
is commutative and pseudocompact, then there are compact Hausdorff spaces $X$ and $K$ and $d_{i} \in \mathbb{N}$ so that $\mathcal{C}(X) \cong \mathfrak{A} \equiv \prod_{\mathcal{U}} \mathbb{C}^{d_{i}} \cong \mathcal{C}(K)$. Since $K$ is totally disconnected, $X$ is totally disconnected.

Alternatively, we can use the fact that being real rank zero is axiomatizable. Since $\prod_{\mathcal{U}} \mathbb{C}^{d_{i}}$ has real rank zero, so does $\mathfrak{A} \cong \mathcal{C}(X)$, so $X$ is totally disconnected.

Likewise, each $\mathbb{C}^{d_{i}}$ is discrete, so the space $K$ satisfying $\prod_{\mathcal{U}} \mathbb{C}^{d_{i}} \cong \mathcal{C}(K)$ has a dense set of isolated points. We will show that this is an axiomatizable property, so the space $X$ with $\mathfrak{A} \cong \mathcal{C}(X)$ must have a dense subset of isolated points.

Thus we have shown that commutative pseudocompact $\mathrm{C}^{*}$-algebras are necessarily of the form $\mathcal{C}(K)$ with $K$ compact, Hausdorff, totally disconnected, with dense isolated points. In fact, all such $\mathrm{C}^{*}$-algebras are pseudocompact.

Corollary 10.3.1 (3.7 in [Hen76]). Let $K$ be any infinite compact Hausdorff space. $\mathcal{C}(K) \equiv \ell^{\infty}$ as real Banach spaces (only equipped with addition and scalar multiplication) if and only if $K$ is totally disconnected with dense isolated points.

In fact, we have the following classification:

Theorem 10.3.2 ([Moo81]). Let $K$ be a compact Hausdorff space. The following are equivalent:

1. $\mathcal{C}(K)$ has a nonstandard hull linearly isometric to a hyperfinite-dimensional Banach space, equivalently, an ultrapower which is linearly isometric to an ultraproduct of finite-dimensional spaces. 
2. $K$ is totally disconnected with a dense subset of isolated points.

3. $\mathcal{C}(K)$ has a nonstandard hull linearly isometric to a nonstandard hull of $\ell^{\infty}$.

4. $\mathcal{C}(K)$ has a nonstandard hull linearly isometric to a $\hat{\ell}^{\infty}(k)$ for $k$ an infinite cardinal.

Henson points out "the results [about real Banach spaces equipped with addition and scalar multiplication] can all be easily extended to include the multiplication of $\mathcal{C}(X) . " \quad$ It is not surprising then that the same criterion holds in the $\mathrm{C}^{*}$-algebraic case.

Recall that a Boolean algebra is a collection of elements equipped with idempotent, commutative binary operators $\wedge$ and $\vee$ and a unary involution operation $\neg$ with distinguished elements 0 and 1 satisfying the following:

$$
\begin{aligned}
a \wedge(b \wedge c)=(a \wedge b) \wedge c & & a \vee(b \vee c)=(a \vee b) \vee c \\
a \wedge b=b \wedge a & & a \vee b=b \vee a \\
a \wedge(a \vee b)=a & & a \vee(a \wedge b) \\
a \wedge 1=a & & a \vee 0=a \\
a \wedge(b \vee c)=(a \wedge b) \vee(a \wedge c) & & a \vee(b \wedge c)=(a \vee b) \wedge(a \vee c) \\
a \wedge(\neg a)=0 & & a \vee(\neg a)=1
\end{aligned}
$$

The classic example is the collection of clopen subsets of a topological space $X$, where 0 and 1 are interpreted as $\emptyset$ and $X$ respectively, $\wedge$ and $\vee$ are $\cap$ and $\cup$, and $\neg$ is complement. 
Let $B$ be a Boolean algebra. We say an element $x \in B$ is an atom if $x \neq 0$ and for all $z<x, z=0$. We say an element $x$ is atomic if every element dominated by $x$ dominates an atom, and an element $y$ is atomless if $y$ dominates no atoms.

Theorem 10.3.3 ([Tar49], [Erš64], [CMS06]). Let $I(B)$ denote the ideal of all elements $x=y \vee z$ where $y$ is atomic and $z$ is atomless. Let $B^{0}=B$, and $B^{n+1}=$ $B^{n} / I\left(B^{n}\right)$ for all $n$. The Tarski invariant is a triple $(p, q, r)$ with $p, q \leq \omega$ and $r \in\{0,1\}$, defined as follows:

$$
\begin{gathered}
p= \begin{cases}\min \left\{n: B^{n+1}=0\right\}, & \text { if it exists } \\
\omega, & \text { otherwise }\end{cases} \\
q= \begin{cases}\sup \left\{n: B^{p} \text { has at least } n \text { atoms }\right\}, & \text { if } p<\omega \\
0, & \text { if } p=\omega\end{cases} \\
r= \begin{cases}1, & \text { if } p<\omega \text { and } B^{p} \text { contains an atomless element } \\
0, & \text { otherwise }\end{cases}
\end{gathered}
$$

Two Boolean algebras are elementarily equivalent if and only if they have the same invariant.

Note that if $K$ is compact Hausdorff, totally disconnected, with dense isolated points, then in $B(K)$ the atoms are the singleton sets containing isolated points, and by the density of the isolated points, every element is atomic. Thus $I(B)=B$ and 
$B^{1}=0$. Thus $p=0$, and $r=0$. If $K$ is infinite, then it has infinitely many isolated points, so $q=\omega$.

Theorem 10.3.4 (3.6 in [Hen76]). Let $X, Y$ be compact Hausdorff spaces with $X$ totally disconnected. Then $\mathcal{C}(X) \equiv \mathcal{C}(Y)$ as real Banach spaces (only equipped with addition and scalar multiplication) if and only if (1) $Y$ is totally disconnected and (2) the Boolean algebras $B(X)$ and $B(Y)$ are elementarily equivalent.

Analogously, in the $\mathrm{C}^{*}$-algebra case we have

Theorem 10.3.5 (5.8 in $[E V 15])$. If $\mathfrak{A}, \mathfrak{B}$ are commutative, real rank zero $\mathrm{C}^{*}$ algebras, so $\mathfrak{A} \cong \mathcal{C}(X)$ and $\mathfrak{B} \cong \mathcal{C}(Y)$ for totally disconnected compact Hausdorff spaces $X, Y$. Then $\mathfrak{A} \equiv \mathfrak{B}$ (as $\mathrm{C}^{*}$-algebras) if and only if $B(X) \equiv B(Y)$ as Boolean algebras.

Corollary 10.3.6 (5.10 in [EV15]). If $X, Y$ are infinite, totally disconnected compact Hausdorff spaces with dense sets of isolated points, then $\mathcal{C}(X) \equiv \mathcal{C}(Y)$.

In particular, a $\mathrm{C}^{*}$-algebra $\mathfrak{A}$ is commutative and pseudocompact if and only if it is *-isomorphic to $\mathcal{C}(K)$ with $K$ compact Hausdorff totally disconnected with dense isolated points. If $\mathfrak{A}$ is infinite-dimensional, commutative, and pseudocompact, then $\mathfrak{A}$ is elementarily equivalent to $\ell_{\infty}(\mathbb{N})=\mathcal{C}(\beta \mathbb{N})$

Now we can axiomatize the class of pseudocompact $\mathrm{C}^{*}$-algebras:

Theorem 10.3.7. $\mathfrak{A}$ is a commutative pseudocompact $\mathrm{C}^{*}$-algebra if and only if it is commutative, unital, real rank zero, and every element can be approximately normed 
by a minimal projection $p$ with $p \mathfrak{A} p=\mathbb{C} p$. The following are a set of axioms for the class of commutative pseudocompact $\mathrm{C}^{*}$-algebras.

1. $\varphi_{u}^{\mathfrak{A}}=\sup _{\|x\|,\|y\| \leq 1}\|x y-y x\|=0$.

2. $\varphi_{c}^{\mathfrak{A}}=\inf _{\|e\| \leq 1} \sup _{\|x\| \leq 1}\|e x-x\|+\|x e-x\|=0$.

3. $\varphi_{\mathrm{rr} 0}^{\mathfrak{A}}=0$.

4. $\sup _{\|x\| \leq 1} \inf _{p \text { a projection }} \sup _{\|y\| \leq 1} \inf _{|\lambda| \leq 1}\|p y p-\lambda p\|+|\|x\|-\|x p\||=0$.

Proof. We know that $\varphi_{u}^{\mathfrak{A}}=0$ if and only if $\mathfrak{A}$ is unital, $\varphi_{c}^{\mathfrak{A}}=0$ if and only if $\mathfrak{A}$ is commutative, and $\varphi_{\text {rr0 }}^{\mathfrak{A}}=0$ if and only if $\mathfrak{A}$ is real rank zero (see $3.6(\mathrm{~b})$ in $\left[\mathrm{FHL}^{+} 16\right]$ ). We also know that a commutative unital $\mathrm{C}^{*}$-algebra $\mathfrak{A} \cong \mathcal{C}(X)$ is real rank zero if and only if $X$ is totally disconnected. Thus we just need to justify that every element being approximately normed by a minimal projection is equivalent to having a dense set of isolated points.

Suppose the underlying compact Hausdorff space $X$ has a dense subset of isolated points. Let $\varepsilon>0$ be given $f \in \mathcal{C}(X)$, then $\{x:|f(x)|>\|f\|-\varepsilon\}$ is a non-empty open set, so it contains an isolated point $x_{0}$, so $p=\chi_{\left\{x_{0}\right\}}$ is a minimal projection (thus $p \mathfrak{A} p=\mathbb{C} p$ ) and $\|p f\|=\left|f\left(x_{0}\right)\right|>\|f\|-\varepsilon$.

Conversely, suppose that $X$ does not have a dense subset of isolated points. Then there is an $x \in X$ disjoint from the closure of the isolated points, so by Urysohn's lemma there is a function that vanishes on the closure of the isolated points but has norm 1 , and this shows that the axiom above has value at least 1 . 
Recall that ideals of $\mathcal{C}(X)$ are of the form $I_{U}=\left\{f|f|_{U^{\mathrm{C}}}=0\right\} \cong \mathcal{C}_{0}(U)$ for open $U \subseteq X$. Thus quotients of $\mathcal{C}(X)$ look like $\mathcal{C}(X) / I_{U} \cong \mathcal{C}\left(U^{\complement}\right)$. Subalgebras of $\mathcal{C}(X)$ are of the form $\mathcal{C}(Y)$ where $Y$ is the image of a quotient map $X \rightarrow Y$.

Example 10.3.8. Let $\mathfrak{C}$ denote the usual Cantor set. Since the Cantor set does not have a dense set of isolated points, $\mathcal{C}(\mathfrak{C})$ is not pseudocompact. In particular we can observe that not all commutative $\mathrm{AF}$ algebras (i.e. those $\mathcal{C}(X)$ with $X$ totally disconnected and metrizable) are pseudocompact. However, the space of convergent sequences of complex numbers $c=\mathcal{C}(\mathbb{N} \cup\{\infty\})$ and the space of bounded sequences $\ell_{\infty}(\mathbb{N})=\mathcal{C}(\beta \mathbb{N})$ are pseudocompact $\mathrm{C}^{*}$-algebras

Since subalgebras of finite-dimensional algebras are finite-dimensional, one might expect subalgebras of pseudocompact algebras to be pseudocompact. However, this is not the case. Let

$$
S_{1}=\{0,1\}, \quad S_{2}=\left\{0, \frac{1}{3}, \frac{2}{3}, 1\right\}, \quad S_{3}=\left\{0, \frac{1}{9}, \frac{2}{9}, \frac{1}{3}, \frac{2}{3}, \frac{7}{9}, \frac{8}{9}, 1\right\}, \quad \ldots
$$

In general, we let $S_{n}=\left\{\frac{k}{3^{n-1}} \mid k \in \mathbb{N}\right\} \cap \mathfrak{C}$ be the set of endpoints of the $n^{\text {th }}$ step of the usual middle-third construction of $\mathfrak{C}$. Consider $X_{0}$ to be the space $\{(0, x) \mid x \in$ $\mathfrak{C}\} \cup\left\{\left(\frac{1}{n}, x\right) \mid n \in \mathbb{N}, x \in S_{n}\right\}$ with the subspace topology from $\mathbb{R}^{2}$. This is compact, Hausdorff, totally disconnected, and has a dense subset of isolated points. This will quotient onto every compact metric space by projecting down onto the Cantor set then following with the surjection that exists by the Hausdorff-Alexandroff theorem, see [Ale27] and [Hau27]. Since subalgebras of $\mathcal{C}\left(X_{0}\right)$ are exactly of the form $\mathcal{C}(X)$ 
where $X$ is a quotient of $X_{0}$, every $\mathcal{C}(X)$ space with $X$ compact metric will be a subalgebra of the pseudocompact $\mathrm{C}^{*}$-algebra $\mathcal{C}\left(X_{0}\right)$.

Conversely, Rudin showed that the class of commutative pseudocompact $\mathrm{C}^{*}$ algebras $\mathcal{C}(X)$ with $X$ compact Hausdorff with no non-empty perfect subsets (like the Cantor set) is closed under subalgebras, see [Rud57]. 


\section{Chapter 11}

\section{Properties of Pseudocompact}

\section{$\mathrm{C}^{*}$-Algebras}

We have seen that pseudocompact and pseudomatricial $\mathrm{C}^{*}$-algebras are unital, tracial, finite (since pseudocompact and pseudomaticial $\mathrm{C}^{*}$-algebras are stable under tensoring by $M_{n}$, they are in fact stably finite), real rank zero, and stable rank one, and (almost) have unitary polar decompositions. It follows that pseudocompact $\mathrm{C}^{*}$ algebras have Lin's property by 6.2 .9 , and the cancellation property, see Proposition 6.5 in [Rie83]. We continue to explore the properties of pseudocompact $\mathrm{C}^{*}$-algebras. These finiteness properties are shared with the unitization of the compacts $\mathcal{K}(\mathcal{H})^{\sim}$, UHF $\mathrm{C}^{*}$-algebras, and $\mathrm{AF}$ algebras. In this Chapter we explore deeper properties which distinguish the pseudocompacts from each of these classes. 


\section{$11.1 \quad$ Unitaries and $K_{1}$}

Note that if $T$ is self-adjoint, then by the continuous functional calculus, $\exp (i T)$ is a unitary. We call these exponential unitaries.

Proposition 11.1.1. Unitaries in $\prod_{\mathcal{U}} M_{k_{i}}$ are exponential unitaries.

Proof. Since the spectrum of a unitary in $M_{n}$ is finite, we can take a branch of the logarithm whose branch cut avoids the spectrum, then use the continuous functional calculus. Thus in $M_{n}$ all unitaries are exponential unitaries $e^{i T}$ for $T$ self-adjoint, and $\|T\| \leq 2 \pi$. See 2.3.1 in [Bla98]. Without loss of generality, unitaries in $\prod_{\mathcal{U}} M_{k_{i}}$ are of the form $\left(u_{i}\right)_{\mathcal{U}}$ where $u_{i} \in M_{k_{i}}$ are unitaries. Thus there are uniformly bounded self-adjoints $t_{i}$ with $u_{i}=\exp \left(i t_{i}\right)$. Thus $\left(u_{i}\right)_{\mathcal{U}}=\left(\exp \left(i t_{i}\right)\right)_{\mathcal{U}}=\exp \left(i\left(t_{i}\right)_{\mathcal{U}}\right)$, because the exponential function is a uniform limit of polynomials on bounded subsets.

Proposition 11.1.2. If every unitary in $\mathfrak{A}^{\mathcal{U}}$ is an exponential unitary, then every unitary in $\mathfrak{A}$ is a norm-limit of exponential unitaries.

Proof. Let $u \in \mathfrak{A}$ be a unitary, then $(u)_{\mathcal{U}} \in \mathfrak{A}^{\mathcal{U}}$ is a unitary, so there is a self-adjoint $\left(t_{i}\right)_{\mathcal{U}}$ with $(u)_{\mathcal{U}}=\exp \left(i\left(t_{i}\right)_{\mathcal{U}}\right)=\left(\exp \left(i t_{i}\right)\right)_{\mathcal{U}}$. Without loss of generality the $t_{i}$ are all self-adjoint, and

$$
\lim _{\mathcal{U}}\left\|u-\exp \left(i t_{i}\right)\right\|=0
$$

So $u$ is a norm-limit of exponential unitaries.

We have shown that the property that every unitary is a norm-limit of exponential unitaries is an axiomatizable property. 
Corollary 11.1.3. If $\mathfrak{A}$ is a pseudocompact $\mathrm{C}^{*}$-algebra, then every unitary is a norm limit of exponential unitaries, and thus $K_{1}(\mathfrak{A})$ is the trivial group.

Proof. The exponential unitaries are contained in $\mathcal{U}_{0}(\mathfrak{A})$, the connected component of the identity in the unitaries, by the homotopy $t \mapsto \exp (i t T)$. Recall that if $\|u-v\|<2$ then $u$ and $v$ are homotopic, see 2.3.1 in [Bla98]. Thus a unitary which is not in $\mathcal{U}_{0}(\mathfrak{A})$ is at least distance two from any exponential unitary. Since the exponential unitaries are dense in the unitary group, we must have $\mathcal{U}_{0}(\mathfrak{A})=\mathcal{U}(\mathfrak{A})$. Thus $K_{1}(\mathfrak{A})$ is trivial.

Question 11.1.4. If the exponential unitaries are dense in the unitary group, are they the entire unitary group?

\subsection{Minimal Projections}

Recall that a non-zero projection $p$ is minimal if for any projection $q, 0 \leq q \leq p$ implies either $q=0$ or $q=p$.

Proposition 11.2.1. A projection $p$ in an ultraproduct is minimal if and only if it has a representative sequence $p=\left(p_{i}\right)_{\mathcal{U}}$ so $\left\{i \mid p_{i}\right.$ is a minimal projection $\} \in \mathcal{U}$.

Proof. We know that any projection $p$ in an ultraproduct has a representative sequence of projections. If $p=\left(p_{i}\right)_{\mathcal{U}}$ is a projection with the $p_{i}$ non-minimal then there are $\mathcal{U}$-many projections $q_{i}$ with $p_{i}>q_{i} \geq 0$, then $\left(p_{i}\right)_{\mathcal{U}}>\left(q_{i}\right)_{\mathcal{U}}$ so $p$ is not minimal. Likewise if $p=\left(p_{i}\right)$ with $\mathcal{U}$-many of the $p_{i}$ minimal then $p$ must be minimal, since if 
$p>q=\left(q_{i}\right)_{\mathcal{U}}$ by Proposition 3.2.1 we can assume $\mathcal{U}$-many of the $q_{i}$ are projections dominated by $p_{i}$, a contradiction.

Given that finite-dimensional $\mathrm{C}^{*}$-algebras are determined by matrix units, and the equations defining matrix units are weakly stable, it is not surprising that projections and partial isometries are our main tool for finding properties of pseudocompact $\mathrm{C}^{*}$ algebras.

In a finite-dimensional $\mathrm{C}^{*}$-algebra $\mathfrak{A}$ represented as a finite direct sum of matrix algebras, a non-zero projection $p$ is minimal if and only if it is rank one, equivalently, $p \mathfrak{A} p=\mathbb{C} p$. The same property holds for pseudocompact $\mathrm{C}^{*}$-algebras.

Proposition 11.2.2. In a pseudocompact $\mathrm{C}^{*}$-algebra $\mathfrak{A}$, a (non-zero) projection $p$ is minimal if and only if $p \mathfrak{A} p=\mathbb{C} p$.

Proof. We call a non-zero projection $p$ with $p \mathfrak{A} p=\mathbb{C} p$ scalar. It is clear that scalar projections must be minimal: if $p$ is not minimal then there is a proper subprojection $0<q<p$ so $\|q\|=1=\|p-q\|$, and then $q=p q p \in p \mathfrak{A} p \backslash \mathbb{C} p$. The converse can fail, for instance the identity in $\mathcal{C}([0,1])$ is minimal but not scalar.

We show that "minimal if and only if scalar" is an axiomatizable property. It is clear this property is preserved by $*$-isomorphisms. If this property holds in each $\mathfrak{A}_{i}$ then it holds in $\prod_{\mathcal{U}} \mathfrak{A}_{i}$ as well: Suppose that $p$ is a minimal projection in $\prod_{\mathcal{U}} \mathfrak{A}_{i}$, then it has a representative sequence of minimal projections, $p=\left(p_{i}\right)_{\mathcal{U}}$, with $p_{i} \mathfrak{A} p_{i}=\mathbb{C} p_{i}$. 
Thus

$$
p\left(\prod_{\mathcal{U}} \mathfrak{A}_{i}\right) p=\prod_{\mathcal{U}} \mathbb{C}\left(p_{i}\right)_{\mathcal{U}}=\mathbb{C} p
$$

If $\mathfrak{A}^{\mathcal{U}}$ has the property "minimal if and only if scalar", then $\mathfrak{A}$ does as well: suppose $p$ is a minimal projection in $\mathfrak{A}$, then $(p)_{\mathcal{U}}$ is a minimal projection in $\mathfrak{A}^{\mathcal{U}}$. Thus $(p)_{\mathcal{U}} \mathfrak{A}^{\mathcal{U}}(p)_{\mathcal{U}}=\mathbb{C}(p)_{\mathcal{U}}$ and we must have $p \mathfrak{A} p=\mathbb{C} p$ by a Kadison Transitivity argument: if $p \mathfrak{A} p \neq \mathbb{C} p$ we can find an element in $p \mathfrak{A} p$ of norm one which is distance one from $\mathbb{C} p$.

This property that minimal projections are scalar is axiomatized by

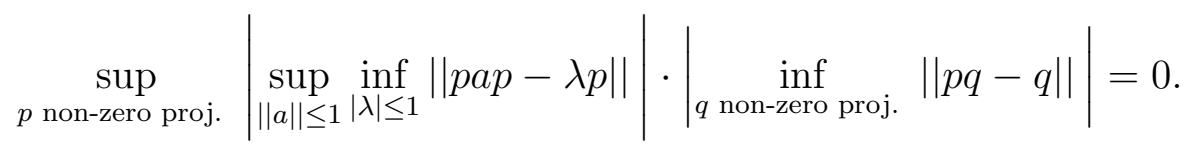

Proposition 11.2.3. In a pseudocompact $\mathrm{C}^{*}$-algebra, every non-zero projection dominates a minimal projection.

Proof. We check that this is an axiomatizable property. It certainly passes through $*-$ isomorphisms. This property passes to ultraproducts: Suppose in each $\mathfrak{A}_{i}$ every nontrivial projection dominates a minimal projection. Then without loss of generality a non-zero projection $p$ in $\prod_{\mathcal{U}} \mathfrak{A}_{i}$ has a representative sequence $\left(p_{i}\right)_{\mathcal{U}}$ where $\mathcal{U}$-many of the $p_{i}$ are non-zero projections. Then the $p_{i}$ dominate some minimal projection $q_{i} \in \mathfrak{A}_{i}$. Thus $\left(p_{i}\right)_{\mathcal{U}}$ dominates the minimal projection $\left(q_{i}\right)_{\mathcal{U}}$.

Finally the property passes to ultraroots: suppose in $\mathfrak{A}^{\mathcal{U}}$ non-trivial projections dominate minimal projections. Given a non-zero projection $p$ in $\mathfrak{A}$ we find a minimal 
projection $q$ dominated by $(p)_{\mathcal{U}}$, then by Proposition 3.2.1 we can find a representative sequence $\left(q_{i}\right)_{\mathcal{U}}$ where $\mathcal{U}$-many of the $q_{i}$ are minimal projections dominated by $p$.

"Every non-zero projection dominates a minimal projection" is an axiomatized by $\sup _{q \text { non-zero proj. }} \quad \inf _{\text {non-zero proj. } v \text { partial isom. }} \sup _{\|a\| \leq 1|\lambda| \leq 1} \inf _{|\lambda|}\|p a p-\lambda p\|+\left\|v^{*} v-p\right\|+\left\|q\left(v v^{*}\right)-v v^{*}\right\|=0$.

In pseudomatricial $\mathrm{C}^{*}$-algebras we can use

$$
\inf _{p \text { non-zero proj. }} \sup _{q \text { non-zero proj. }} \inf _{\text {partial isom. }}\left\|v^{*} v-p\right\|+\left\|q\left(v v^{*}\right)-v v^{*}\right\|=0
$$

which says there is a non-zero projection subequivalent to every other non-zero projection.

Notice that this is quite different than the tracial von Neumann algebra case considered in Section 4 of [FH11], Proposition 6.5 in [FHS14a], and Section 5 of [FHS14b].

Since UHF algebras lack minimal projections, we observe:

Proposition 11.2.4. UHF algebras are not pseudocompact.

\subsection{Approximate Fractions of Projections}

Let $n$ and $d$ be natural numbers. Then we can write $n=k d+r$ where $0 \leq r<d$. This means the identity in $M_{n}$ is the orthogonal sum of $d$ Murray-von Neumann equivalent projections (each of rank $k$ ) and fewer than $d$ minimal, (i.e. rank one) projections. We can similarly decompose the identity in any pseudocompact $\mathrm{C}^{*}$-algebra: 
Proposition 11.3.1. Let $d \geq 1$ be a natural number.

1. In a pseudomatrical $\mathrm{C}^{*}$-algebra, the identity can be written as a sum of $d$ orthogonal Murray-von Neumann equivalent projections plus no more than $d-1$ orthogonal minimal or zero projections.

2. In a pseudocompact $\mathrm{C}^{*}$-algebra, the identity can be written as a sum of $d$ orthogonal Murray-von Neumann equivalent projections plus an orthogonal abelian projection.

Proof. This is an axiomatizable property. Here is the case $d=2$ :

$$
\inf _{v \text { a partial isom. }} \inf _{\text {a proj }}\left\|I-\left(v^{*} v+v v^{*}+p\right)\right\|+\sup _{\|x\|,\|y\| \leq 1} \| \text { pxpyp }- \text { pypxp } \|=0 .
$$

When we write the identity as $I=\sum_{i=1}^{d} p_{i}+r$ where the $p_{i}$ are orthogonal, pairwise Murray-von Neumann equivalent projections and $r$ is a sum of $d-1$ or fewer orthogonal minimal projections, we call the $p_{i}$ an approximate $1 / d^{\text {th }}$ of the identity.

We can do this sort of decomposition with any projection, of course.

Proposition 11.3.2. The unitization of the compact operators on an infinite-dimensional Hilbert space is not pseudocompact.

Proof. In the unitization of the compacts, projections are either finite rank or cofinite-rank, so there are no approximate halves of the identity.

In pseudomatricial $\mathrm{C}^{*}$-algebras, it is natural to think of the number of orthogonal minimal projections left over after splitting the identity into $d$ orthogonal Murrayvon Neumann equivalent projections as the remainder mod $d$. In particular, if the 
identity in two pseudomatricial algebras have different remainders mod $d$, then the $\mathrm{C}^{*}$-algebras are not elementarily equivalent and thus not $*$-isomorphic.

Corollary 11.3.3. There are uncountably many *-isomorphism classes of separable pseudomatrical $\mathrm{C}^{*}$-algebras.

It seems possible that these remainders $\bmod d$ determine a pseudomatricial $\mathrm{C}^{*}$ algebra up to elementary equivalence:

\section{Conjecture 11.3.4.}

$\prod_{\mathcal{U}} M_{n_{i}} \equiv \prod_{\mathcal{V}} M_{m_{j}} \quad$ if and only if $\quad \lim _{\mathcal{U}} n_{i} \bmod d=\lim _{\mathcal{V}} m_{i} \bmod d \quad$ for all $d \in \mathbb{N}$.

This is probably equivalent to the conjecture "Two pseudomatricial $\mathrm{C}^{*}$-algebras are elementarily equivalent if and only if they have elementarily equivalent ordered $K_{0}$ groups with distinguished order units". The $K_{0}$ group of a pseudomatrical C*-algebra is an ordered abelian group, and they are regularly discrete since the equivalence class of a minimal projection is a smallest positive element in the $K_{0}$ group, and there are $p$ equivalence classes of projections modulo $p$ for all primes $p$. By Theorem 4.7 in [RZ60], all regularly discrete abelian groups are elementarily equivalent but the language does not include constants for the order unit or the definable minimal projection.

Calculating the remainders mod $d$ generally requires knowing a lot about the ultrafilter in question. One can simplify things by making sure the pseudomatricial $\mathrm{C}^{*}$-algebras have matrix units of all orders. A nice test case is 
Question 11.3.5. Let $\mathcal{U}$ be a free ultrafilter on $\mathbb{N}$. Is it the case that

$$
\prod_{\mathcal{U}} M_{n !} \quad \text { and } \quad \prod_{\mathcal{U}} M_{(n !)^{2}}
$$

are elementarily equivalent?

In fact, under the continuum hypothesis an affirmative answer would imply the two algebras are $*$-isomorphic. If two ultraproducts are saturated and elementarily equivalent, a back-and-forth style argument allows one to construct an $*$-isomorphism. See 4.13 in [FHS14a].

\subsection{The Dixmier Property}

Definition 11.4.1. We say a unital $\mathrm{C}^{*}$-algebra $\mathfrak{A}$ with center $\mathcal{Z}(\mathfrak{A})$ has the Dixmier property if for all $a \in \mathfrak{A}$, the norm-closed convex hull of the unitary orbit of $\mathfrak{A}$ contains a central element. Writing $\mathcal{U}(a)=\left\{u^{*} a u: u \in \mathcal{U}(\mathfrak{A})\right\}$ for the unitary orbit of $a, \mathfrak{A}$ has the Diximier property if

$$
\text { for all } a \in \mathfrak{A}, \quad \overline{\operatorname{conv}(\mathcal{U}(\mathfrak{A}))} \cap \mathcal{Z}(\mathfrak{A}) \neq \emptyset \text {. }
$$

Theorem 11.4.2 (5.3 in [Dix96], III.2.5.18, 19 in [Bla06]). Let $\mathfrak{M}$ be a von Neumann algebra. For every self-adjoint $x \in M$, there is a $u \in \mathcal{U}(M)$ and a $z \in \mathcal{Z}(\mathfrak{M})$ with

$$
\left\|\frac{1}{2}\left(x+u^{*} x u\right)-z\right\| \leq \frac{3}{4}\|x\| .
$$

By iterating this result, one can see all finite von Neumann algebras have the Dixmier property. The important observation for us is that the number of terms 
needed for a convex combination to get within $\varepsilon$ of the center is bounded as a function of $\varepsilon$, independent of the element $x$.

Proposition 11.4.3. Central projections are the zero-set of the weakly-stable relation

$$
\varphi(x)=\left\|x-x^{*}\right\|+\left\|x-x^{2}\right\|+\sup _{y}\|[x, y]\| .
$$

So the set of central projections is definable.

Proof. Certainly this is zero for any central projection. It is also at least 1 for noncentral projections. If $p$ is a projection and not central, then there is an irreducible representation $\pi: \mathfrak{A} \rightarrow \mathcal{B}(\mathcal{H})$ so $\pi(p)$ is not zero or the identity. By a Kaplansky density argument there is a $y,\|y\| \leq 2$ so $\|[y, p]\| \geq\|[\pi(y), \pi(x)]\| \geq 1$.

In particular, we can quantify over the central projections.

Proposition 11.4.4. Pseudocompact $\mathrm{C}^{*}$-algebras have Dixmier's property.

Proof. For each $n$ pick $K_{n} \in \mathbb{N}$ satisfying $(3 / 4)^{K_{n}}<\frac{1}{2 n}$. Take $\left\{\lambda_{1}, \ldots, \lambda_{D_{n}}\right\}$ to be a $\frac{1}{2 n}$ net in the unit disk in the complex numbers. Now for any finite-dimensional $\mathrm{C}^{*}$-algebra, since it is a finite von Neumann algebra, for all $n$, and all self-adjoint $x$, there is a central element $z$ so that

$$
\sup _{x} \inf _{u_{1}, \ldots, u_{K_{n}}} \text { unitaries }\left\|\sum_{1}^{K_{n}} \frac{1}{2^{K_{n}}} u_{i}^{*} x u_{i}-z\right\|<\frac{1}{2 n} .
$$

Since we are in a finite-dimensional $\mathrm{C}^{*}$-algebra, the central element $z$ is a linear combination of central projections, $z=\sum_{1}^{d} \mu_{i} q_{i}$, which is within distance $\frac{1}{2 n}$ to a 
linear combination of the form $\sum_{1}^{D_{n}} \lambda_{i} p_{i}$ where the $p_{i}$ are central projections. Thus for all $n$, in every finite-dimensional $\mathrm{C}^{*}$-algebra,

$$
\sup _{x} \inf _{u_{1}, \ldots, u_{K_{n}} \text { unitaries }} \inf _{p_{1}, \ldots, p_{D_{n}}} \inf _{\text {central projs. } .} \inf _{\lambda_{1}, \ldots, \lambda_{D_{n}} \in \overline{\mathbb{D}}}\left\|\sum_{1}^{K_{n}} \frac{1}{2^{K_{n}}} u_{i}^{*} x u_{i}-\sum_{1}^{D_{n}} \lambda_{j} p_{j}\right\| \leq \frac{1}{n} .
$$

In particular, we have the same inequality for every pseudocompact $\mathrm{C}^{*}$-algebra.

Comment: this implies that the trace on a pseudomatricial $\mathrm{C}^{*}$-algebra is definable. See section 3.4 and 7.2 in $\left[\mathrm{FHL}^{+} 16\right]$.

Observe that it is not clear if an ultraproduct of $\mathrm{C}^{*}$-algebras with Dixmier's property must have Dixmier's property, since the number of terms needed to approximate a given element may not be bounded. It is also not clear that if $\mathfrak{A}^{\mathcal{U}}$ has Dixmier's property then $\mathfrak{A}$ has Dixmier's property, since although given $a \in \mathfrak{A}$ we can find a convex combination of unitaries and a central element $z \in \mathfrak{A}^{\mathcal{U}}$ so that

$$
\left\|\sum_{j=1}^{n} u_{j}(a)_{\mathcal{U}} u_{j}-z\right\|<\varepsilon
$$

Taking representative sequences $u_{j}=\left(u_{j}(i)\right)_{\mathcal{U}}$ and $z=\left(z_{i}\right)_{\mathcal{U}}$, we have

$$
\lim _{\mathcal{U}}\left\|\sum_{j=1}^{n} u_{j}(i) a u_{j}(i)-z_{i}\right\|<\varepsilon
$$

Although the $z_{i}$ are nearly central in that for all $a \in \mathfrak{A}, \lim _{\mathcal{U}}\left\|z_{i} a-a z_{i}\right\|=0$, the problem is that we do not know that $z_{i}$ is close to a central element in $\mathfrak{A}$. However, this is not an issue for pseudocompact $\mathrm{C}^{*}$-algebras.

Let $\mathfrak{A}$ be a $\mathrm{C}^{*}$-algebra with center $\mathcal{Z}(\mathfrak{A})$. For each $a \in \mathfrak{A}$ we have the inner derivation induced by $\mathfrak{A}, \Delta_{a}: x \mapsto a x-x a$. For every $a \in \mathfrak{A}$ a finite-dimensional 
$\mathrm{C}^{*}$-algebra, we have $\left\|\Delta_{a}\right\|=2 \operatorname{dist}(a, \mathcal{Z}(\mathfrak{A}))$, see [Zsi73]. Archbold defined $K(\mathfrak{A})=$ $\inf \left\{K: \operatorname{dist}(a, \mathcal{Z}(\mathfrak{A})) \leq K\left\|\Delta_{a}\right\| \forall a \in \mathfrak{A}\right\}$, see [Arc78]

Proposition 11.4.5 ([Rin78]). If $\mathfrak{A}$ has the Dixmier property then $K(\mathfrak{A}) \leq 1$.

Proof. For all $x, y \in \mathfrak{A}$, for all $z \in \mathcal{Z}(\mathfrak{A})$,

$$
\left\|\Delta_{x}(y)\right\|=\|x y-y x\|=\|x y-z y+y z-y x\| \leq 2\|y\|\|x-z\| .
$$

Thus

$$
\left\|\Delta_{x}\right\|=\sup _{\|y\|=1}\left\|\Delta_{x}(y)\right\| \leq 2 \operatorname{dist}(x, \mathcal{Z}(\mathfrak{A})) .
$$

On the other hand, since

$$
\left\|x-u^{*} x u\right\|=\|u x-x u\|=\left\|\Delta_{x}(u)\right\| \leq\left\|\Delta_{x}\right\|,
$$

we have that the convex hull of the unitary orbit of $x$ is contained in the ball of radius $\left\|\Delta_{x}\right\|$ centered at $x$, but by Dixmier's property this ball contains a central element, so

$$
\operatorname{dist}(x, \mathcal{Z}(\mathfrak{A})) \leq\left\|\Delta_{x}\right\| \leq 2 \operatorname{dist}(x, \mathcal{Z}(\mathfrak{A})) .
$$

This shows that in a $\mathrm{C}^{*}$-algebra $\mathfrak{A}$ with the Dixmier property then the center is a definable set: it is the zero set of

$$
\varphi_{\mathcal{Z}}(z)=\sup _{\|a\| \leq 1}\|z a-a z\|
$$

Corollary 11.4.6. Let $\mathfrak{A}$ be a pseudocompact $\mathrm{C}^{*}$-algebra. Then $K(\mathfrak{A}) \leq 1$ and the inner derivations on $\mathfrak{A}$ are point-norm closed in the space of all derivations on $\mathfrak{A}$. 
Proof. This follows from the Dixmier property and Theorem 5.3 in [KLR67].

Proposition 11.4.7. If $\mathfrak{A}_{i}$ is pseudocompact for all $i \in I$, and $\mathcal{U}$ is an ultrafilter on $I$, we have $\mathcal{Z}\left(\prod_{\mathcal{U}} \mathfrak{A}_{n}\right)=\prod_{\mathcal{U}} \mathcal{Z}\left(\mathfrak{A}_{n}\right)$. In particular, the center of a pseudocompact $\mathrm{C}^{*}$-algebra is pseudocompact, and pseudomatrical $\mathrm{C}^{*}$-algebras have trivial center.

Proof. If $\mathfrak{A}$ pseudocompact, there are ultrafilters $\mathcal{V}$ and $\mathcal{U}$ and finite-dimensional $\mathrm{C}^{*}$-algebras $F_{i}$ satisfying

$$
\mathfrak{A}^{\mathcal{V}} \cong\left(\prod_{i \rightarrow \mathcal{U}} F_{i}\right)^{\mathcal{V}}
$$

Then we have

$$
(\mathcal{Z}(\mathfrak{A}))^{\mathcal{V}}=\mathcal{Z}\left(\mathfrak{A}^{\mathcal{V}}\right) \cong \mathcal{Z}\left(\left(\prod_{\mathcal{U}} F_{i}\right)^{\mathcal{V}}\right)=\left(\prod_{\mathcal{U}} \mathcal{Z}\left(F_{i}\right)\right)^{\mathcal{V}}
$$

The middle isomorphism of course holds. The right equality also holds: the containment $\supseteq$ is clear. Conversely, if $\left(\left(a_{i}^{(j)}\right)_{i \rightarrow \mathcal{U}}\right)_{j \rightarrow \mathcal{V}} \notin\left(\prod_{\mathcal{U}} \mathcal{Z}\left(F_{i}\right)\right)^{\mathcal{V}}$, then

$$
\operatorname{dist}\left(\left(\left(a_{i}^{(j)}\right)_{i \rightarrow \mathcal{U}}\right)_{j \rightarrow \mathcal{V}},\left(\prod_{\mathcal{U}} \mathcal{Z}\left(F_{i}\right)\right)^{\mathcal{V}}\right)=\varepsilon>0 .
$$

Then there is a set $V \in \mathcal{V}$ so for all $j \in V$,

$$
\operatorname{dist}\left(\left(a_{i}^{(j)}\right)_{i \rightarrow \mathcal{U}}, \prod_{\mathcal{U}} \mathcal{Z}\left(F_{i}\right)\right) \geq \frac{\varepsilon}{2} .
$$

Then there is a set $U_{j} \in \mathcal{U}$ so that for all $i \in U_{j}$,

$$
\operatorname{dist}\left(a_{i}^{(j)}, \mathcal{Z}\left(F_{i}\right)\right) \geq \frac{\varepsilon}{4}
$$

But this means

$$
\left\|\Delta_{a_{i}^{(j)}}\right\|=\sup _{\|b\| \leq 1}\left\|\left[a_{i}^{(j)}, b\right]\right\|=2 \operatorname{dist}\left(a_{i}^{(j)}, \mathcal{Z}\left(F_{i}\right)\right) \geq \frac{\varepsilon}{2}
$$


So there is some $b_{i}^{(j)} \in F_{i}$ of norm no more than one so $\left\|\left[a_{i}^{(j)}, b_{i}^{(j)}\right]\right\| \geq \varepsilon / 4$. Consider $\left(\left(b_{i}^{(j)}\right)_{i \rightarrow \mathcal{U}}\right)_{j \rightarrow \mathcal{V}} \in\left(\prod_{i \rightarrow \mathcal{U}} F_{i}\right)^{\mathcal{V}}$ (it is defined almost everywhere and has norm no more than one), then

$$
\left\|\left[\left(\left(a_{i}^{(j)}\right)_{i \rightarrow \mathcal{U}}\right)_{j \rightarrow \mathcal{V}},\left(\left(b_{i}^{(j)}\right)_{i \rightarrow \mathcal{U}}\right)_{j \rightarrow \mathcal{V}}\right]\right\|=\lim _{j \rightarrow \mathcal{V}} \lim _{i \rightarrow \mathcal{U}}\left\|\left[a_{i}^{(j)}, b_{i}^{(j)}\right]\right\| \geq \varepsilon / 4 .
$$

so $\left(\left(a_{i}^{(j)}\right)_{i \rightarrow \mathcal{U}}\right)_{j \rightarrow \mathcal{V}} \notin \mathcal{Z}\left(\left(\prod_{\mathcal{U}} F_{i}\right)^{\mathcal{V}}\right)$. Finally, the same argument shows that $\mathcal{Z}\left(\mathfrak{A}^{\mathcal{V}}\right)=$ $(\mathcal{Z}(\mathfrak{A}))^{\mathcal{V}}$ for pseudocompact $\mathfrak{A}$, since they have the Dixmier property and thus have $K(\mathfrak{A}) \leq 1$, so almost central elements are near central elements.

Example 11.4.8. $K(\mathfrak{A})$ can be infinite even for AF-algebras, and the containment $\mathcal{Z}\left(\prod_{\mathcal{U}} \mathfrak{A}_{n}\right) \supseteq \prod_{\mathcal{U}} \mathcal{Z}\left(A_{n}\right)$ can be proper. Example 6.2 in [Arc78] gives a unital AF algebra $\mathfrak{A}$ with trivial center and a sequence $a_{n} \in \mathfrak{A}$ with $\lim _{n \rightarrow \infty}\left\|\Delta_{a_{n}}\right\|=0$ but $\operatorname{dist}\left(a_{n}, \mathcal{Z}(\mathfrak{A})\right)=1$ for all $n$. In particular, if $\mathcal{U}$ is a free ultrafilter on $\mathbb{N}$, then $\left(a_{n}\right)_{\mathcal{U}} \in \mathcal{Z}\left(\mathfrak{A}^{\mathcal{U}}\right)$ but not in $(\mathcal{Z}(\mathfrak{A}))^{\mathcal{U}}$

We mention that the literature suggests many connections between norms of inner derivations, Dixmier's property, primitive ideal spaces, multiplier algebras, central sequences, and relative commutants. These certainly deserve further study. 


\section{Chapter 12}

\section{Properties of Pseudomatricial}

\section{$\mathrm{C}^{*}$-Algebras}

Now we will focus our attention on the smaller class of pseudomatricial $\mathrm{C}^{*}$-algebras.

\subsection{Trivial Centers}

Pseudomatrical $\mathrm{C}^{*}$-algebras are easily distinguished from other pseudocompact algebras since they have trivial centers:

Proposition 12.1.1. A pseudocompact $\mathrm{C}^{*}$-algebra $\mathfrak{A}$ is pseudomatricial if and only if $\mathcal{Z}(\mathfrak{A})=\mathbb{C} I$.

Proof. The pseudocompact $\mathrm{C}^{*}$-algebras which are not pseudomatricial will be elementarily equivalent to $\prod_{\mathcal{U}} F_{i}$ where the $F_{i}$ are finite-dimensional and $\mathcal{U}$-many of the 
$F_{i}$ are non-trival direct sums of matrix algebras. In particular, $\mathcal{U}$-many of the $F_{i}$ have non-trivial central projections, axiomatized by

$$
\inf _{p \text { a proj. }}|\|p-I\|-1|+|\|p\|-1|+\sup _{\|x\| \leq 1}\|p x-x p\| .
$$

\subsection{Highly Irreducible Elements}

We let $\operatorname{Red}_{n}$ denote the matrices in $M_{n}(\mathbb{C})$ which have a non-trivial reducing subspace. The following result due to Herrero and Szarek, and the technique used to prove it, is very similar to the result and technique of an oft-ignored paper by von Neumann, [vN42]. Neither technique is constructive. The von Neumann paper anticipated many of the ideas behind continuous logic and pseudocompact $\mathrm{C}^{*}$-algebras.

Theorem 12.2.1 ([HS86]). There is an $\varepsilon>0$ so for all $n \geq 2$, there is an $A \in M_{n}(\mathbb{C})$ with $\|A\| \leq 1$ and $\operatorname{dist}\left(A, \operatorname{Red}_{n}\right) \geq \varepsilon$.

Notice that if $B$ is an matrix with a (necessarily closed) reducing subspace $\mathcal{K}$, letting $P_{\mathcal{K}}$ be the projection onto $\mathcal{K}$ and $P_{\mathcal{K}}^{\perp}=1-P_{\mathcal{K}}$ be the projection onto $\mathcal{K}^{\perp}$, we have $\left[B, P_{\mathcal{K}}\right]=0$. For a non-trivial projection $P, B=P A P-P^{\perp} A P^{\perp} \in \operatorname{Red}_{n}$, and

$$
\|A-B\|=\left\|P A P^{\perp}+P^{\perp} A P\right\|=\left\|P A P^{\perp}-P^{\perp} A P\right\|=\|P A-A P\| .
$$

Thus

$$
\operatorname{dist}\left(A, \operatorname{Red}_{n}\right)=\inf _{P \neq 0, I}\|[A, P]\|
$$


Corollary 12.2.2. There is an $\varepsilon>0$ so that the sentence $\varphi_{\text {irr }}$ given by

$$
\inf _{\|a\| \leq 1} \sup _{p \text { non-trivial proj. }}\|[a, p]\|
$$

has $\varphi_{i r r}^{M_{n}}>\varepsilon$ for all $n \geq 2$.

Voicluescu's theorem [Voi76] says that $\varphi_{\text {irr }}$ evaluates to zero on $\mathcal{B}\left(\ell^{2}(\mathbb{N})\right)$, in other words, the reducible operators are dense. Williams [Wil70] showed conversely that the closure of the operators with a finite-dimensional reducing subspace is nowhere dense, so Voicluescu's result relies on infinite-dimensionality.

\subsection{Commutators}

An element $x$ in a $\mathrm{C}^{*}$-algebra is called a self-commutator if there is an element $a$ with $x=a^{*} a-a a^{*}=\left[a^{*}, a\right]$. Clearly, self-commutators are self-adjoint and trace-zero. In fact, for matrices these are equivalent:

Proposition 12.3.1 ([Tho58]). A matrix is a self-commutator if and only if it is self-adjoint and trace-zero.

In fact, we can modify Thompson's proof to sharpen the result:

Proposition 12.3.2. A matrix $X \in M_{n}(\mathbb{C})$ is self-adjoint and trace zero if and only if there is an $A \in M_{n}(\mathbb{C})$ with $\|A\| \leq \sqrt{2\|X\|}$ and $X=A^{*} A-A A^{*}$.

Proof. Suppose $X$ is self-adjoint and trace-zero. Since $X$ is diagonalizable, so there is a unitary $U$ and a diagonal matrix $D$ so that $X=U^{*} D U$. It suffices to show 
there is a matrix $B$ with $D=\left[B^{*}, B\right]$ and $\|B\| \leq \sqrt{2\|X\|}$ since we can then take $A=U^{*} B U$. Let $\left\{d_{i}\right\}$ be the diagonal entries of $D$, then $\|X\|=\|D\|=\max _{i}\left|d_{i}\right|$. By replacing $D$ with $-D$ we can assume $\|D\|=\max _{i} d_{i}$ by considering $A A^{*}-A^{*} A$ instead. We can easily rearrange the diagonal entries $d_{1}, \ldots d_{n}$ of the matrix $d$ by conjugating by the appropriate permutation matrices. Since $X$ is self-adjoint, all the $d_{i}$ are real numbers, and since $\tau(X)=0$, we have $\sum_{i} d_{i}=0$. Claim: we can reindex the $d_{i}$ to $e_{i}$ so that for $1 \leq k \leq n$,

$$
0 \leq \sum_{i=1}^{k} e_{i} \leq 2\|X\|
$$

We do this iteratively. We take $e_{1}=d_{i}$ so that $d_{i}=\|X\|$. Now we assume that $e_{1}, \ldots, e_{m}$ have been picked from the $d_{i}$ so the partial sums have

$$
0 \leq \sum_{i=1}^{k} e_{i} \leq 2\|X\| \quad \text { for } \quad 1 \leq k \leq m
$$

If there are no positive $d_{i}$ remaining we take take $e_{m+1}, \ldots, e_{n}$ to be the unused non-positive $d_{i}$ in any order, since the total sum is zero this choice will fulfill our requirement. Otherwise, suppose $\sum_{i=1}^{m} e_{i} \leq\|X\|$. Then we can select $e_{m+1}$ to be a positive $d_{i}$, and since $d_{i} \leq\|X\|$ this will fulfill our requirement. If on the other hand we had $\sum_{i=1}^{m} e_{i}>\|X\|$, since the total sum of the $d_{i}$ is zero, we can take $e_{m+1}$ to be a negative $d_{i}$, and since $\left|d_{i}\right| \leq\|X\|$ the next partial sum still fulfills our requirement. Thus we have rearranged the $d_{i}$ to $e_{i}$ so that all the partial sums are non-negative and no larger than $2\|X\|, e_{1} \geq 0$ and $e_{n} \leq 0$. Now we define

$$
b_{j}=\sqrt{\sum_{i=1}^{j} e_{i}} \quad \text { for } \quad 1 \leq j \leq n-1 .
$$


Notice that

$$
-\left|b_{n-1}\right|^{2}=-\sum_{i=1}^{n-1} e_{i}=e_{n} .
$$

Consider

Then it is straightforward to calculate

$$
B=\left[\begin{array}{ccccc}
0 & & & & 0 \\
b_{1} & 0 & & & \\
& b_{2} & 0 & & \\
& & \ddots & \ddots & \\
& & & & \\
& & & b_{n-1} & 0
\end{array}\right]
$$

$$
\begin{aligned}
B^{*} B-B B^{*} & =\operatorname{diag}\left(\left|b_{1}\right|^{2},\left|b_{2}\right|^{2}-\left|b_{1}\right|^{2}, \ldots,\left|b_{n-1}\right|^{2}-\left|b_{n-2}\right|^{2},-\left|b_{n-1}\right|^{2}\right) \\
& =\operatorname{diag}\left(e_{1},\left(e_{1}+e_{2}\right)-e_{1}, \ldots, \sum_{i=1}^{n-1} e_{i}-\sum_{i=1}^{n-2} e_{i},-\sum_{i=1}^{n-1} e_{i}\right) \\
& =\operatorname{diag}\left(e_{1}, e_{2}, \ldots, e_{n-1}, e_{n}\right)
\end{aligned}
$$

which is unitarily equivalent to $D$ and thus to $X$. Finally

$$
\begin{aligned}
\|B\|^{2}=\left\|B^{*} B\right\| & =\left\|\operatorname{diag}\left(\left|b_{1}\right|^{2},\left|b_{2}\right|^{2}, \ldots,\left|b_{n-1}\right|^{2}\right)\right\| \\
& =\max _{i}\left|b_{i}\right|^{2}=\max _{i}\left(\sum_{j=1}^{i} e_{j}\right) \leq 2\|X\| .
\end{aligned}
$$

Proposition 12.3.3. In $\prod_{\mathcal{U}} M_{n_{i}}$, elements are self-commutators if and only if they are self-adjoint and trace-zero. Recall the trace on the ultraproduct is given by $\tau\left(\left(x_{i}\right)_{\mathcal{U}}\right)=\lim _{\mathcal{U}} \tau_{i}\left(x_{i}\right)$ where $\tau_{i}$ is the unique normalized trace on $M_{n_{i}}$.

Proof. Suppose $\left(x_{i}\right)_{\mathcal{U}}^{*}=\left(x_{i}\right)_{\mathcal{U}}$ and $\lim _{\mathcal{U}} \tau_{i}\left(x_{i}\right)=0$, without loss of generality we can assume $x_{i}=x_{i}^{*}$ for all $i$. Let $y_{i}=x_{i}-\tau_{i}\left(x_{i}\right) I_{i}$, then $y_{i}$ is self-adjoint (since $x_{i}$ is self- 
adjoint, $\tau_{i}\left(x_{i}\right)$ is real) and has trace zero, so $y_{i}=\left[a_{i}^{*}, a_{i}\right]$ for some $a_{i}$ with uniformly bounded norm. Thus

$$
\left(x_{i}\right)_{\mathcal{U}}=\left(y_{i}\right)_{\mathcal{U}}=\left(\left[a_{i}^{*}, a_{i}\right]\right)_{\mathcal{U}}=\left[\left(a_{i}\right)_{\mathcal{U}}^{*},\left(a_{i}\right)_{\mathcal{U}}\right]
$$

Proposition 12.3.4. If $\mathfrak{A}^{\mathcal{U}}$ has the property that self-adjoint trace-zero elements are commutators, then in $\mathfrak{A}$ self-adjoint trace-zero elements are norm-limits of commutators.

Proof. Suppose that $a \in \mathfrak{A}$ is self-adjoint and trace-zero. Then $(a)_{\mathcal{U}} \in \mathfrak{A}^{\mathcal{U}}$ is selfadjoint and trace-zero. Then

$$
(a)_{\mathcal{U}}=\left[\left(b_{i}\right)_{\mathcal{U}}^{*},\left(b_{i}\right)_{\mathcal{U}}\right]=\left(\left[b_{i}^{*}, b_{i}\right]\right)_{\mathcal{U}}
$$

Thus $\lim _{\mathcal{U}}\left\|a-\left[b_{i}^{*}, b_{i}\right]\right\|=0$ and $a$ is a limit of commutators.

It is natural to ask if a norm-limit of self-commutators is a self-commutator, and that is in fact the case:

Proposition 12.3.5 (Theorem 3 in [Wil70]). The set of self-commutators is normclosed in $\mathcal{B}(\mathcal{H})$.

Corollary 12.3.6. An element in a separable pseudomatricial $\mathrm{C}^{*}$-algebra is a selfcommutator if and only if it is self-adjoint and trace-zero.

There is a stronger result for matrices: 
Theorem 12.3.7 ([Sho37], [AM57]). In $M_{n}$, a matrix has trace zero if and only if it is a commutator. That is, $\tau(A)=0$ if and only if there are matrices $B$ and $C$ such that $A=[B, C]$.

Question 12.3.8. In a pseudomatricial $\mathrm{C}^{*}$-algebra, does trace-zero imply norm-limit of commutators?

One way to do this would be to show that if $A$ is trace-zero matrix then we can find $B, C$ so that $A=[B, C]$ and $\|B\|,\|C\|$ are bounded by a function of $\|A\|$. This question has been studied:

Theorem 12.3.9 ([JOS13]). For all $\varepsilon>0$, there is a constant $K_{\varepsilon}$ so that if an $m \times m$ matrix has trace zero, then there are $m \times m$ matrices $B$ and $C$ with $A=[B, C]$ and $\|B\| \cdot\|C\| \leq K_{\varepsilon} m^{\varepsilon}\|A\|$. Moreover, $B$ can be taken as normal.

Whether the norm bound on $B$ and $C$ can be taken to be independent of the dimension $m$ remains open, so we do not know if trace-zero elements in an ultraproduct of matrix algebras can be written as a commutator. We have the next best thing, however:

Proposition 12.3.10. If $\left(a_{i}\right)_{\mathcal{U}} \in \prod_{\mathcal{U}} M_{n_{i}}$ has trace zero, then it is a linear combination of two commutators.

Proof. Since $\lim _{\mathcal{U}} \tau_{i}\left(a_{i}\right)=0$, we have

$$
\left(a_{i}\right)_{\mathcal{U}}=\left(a_{i}-\tau_{i}\left(a_{i}\right) I\right)_{\mathcal{U}}
$$


Since $\Re\left(a_{i}-\tau_{i}\left(a_{i}\right) I\right)$ is self-adjoint and trace zero, there are $b_{i}, c_{i} \in M_{n_{i}}$ with uniformly bounded norm so that $\Re\left(a_{i}-\tau_{i}\left(a_{i}\right) I\right)=\left[b_{i}, c_{i}\right]$, and similarly there are $d_{i}, e_{i} \in M_{n_{i}}$ with $\Im\left(a_{i}-\tau_{i}\left(a_{i}\right) I\right)=\left[d_{i}, e_{i}\right]$. Thus

$$
\left(a_{i}\right)_{\mathcal{U}}=\left(\left[b_{i}, c_{i}\right]+i\left[d_{i}, e_{i}\right]\right)_{\mathcal{U}}=\left[\left(b_{i}\right)_{\mathcal{U}},\left(c_{i}\right)_{\mathcal{U}}\right]+i\left[\left(d_{i}\right)_{\mathcal{U}},\left(e_{i}\right)_{\mathcal{U}}\right]
$$

Conversely, we have

Proposition 12.3.11. Suppose in $\mathfrak{A}^{\mathcal{U}}$ trace-zero elements are a linear combination of two commutators (where the trace is the usual limit trace). Then if $a$ is trace-zero in $\mathfrak{A}$ is it a norm-limit of linear combinations of two commutators.

Proof. Let $a \in \mathfrak{A}$ with $\tau(a)=0$, then $(a)_{\mathcal{U}}$ has $\tau_{\mathcal{U}}\left((a)_{\mathcal{U}}\right)=\lim _{\mathcal{U}} \tau(a)=0$, so there are $\left(b_{i}\right)_{\mathcal{U}},\left(c_{i}\right)_{\mathcal{U}},\left(d_{i}\right)_{\mathcal{U}},\left(e_{i}\right)_{\mathcal{U}}$ so that

$$
(a)_{\mathcal{U}}=\lambda_{i}\left[\left(b_{i}\right)_{\mathcal{U}},\left(c_{i}\right)_{\mathcal{U}}\right]+\mu_{i}\left[\left(d_{i}\right)_{\mathcal{U}},\left(e_{i}\right)_{\mathcal{U}}\right]
$$

Thus

$$
\lim _{\mathcal{U}}\left\|a-\left(\lambda_{i}\left[b_{i}, c_{i}\right]+\mu_{i}\left[d_{i}, e_{i}\right]\right)\right\|=0
$$

Therefore $a$ is a norm-limit of linear combinations of two commutators.

Corollary 12.3.12. In a pseudomatricial $\mathrm{C}^{*}$-algebra, an element is trace-zero if and only if it is the norm-limit of a linear combination of two commutators.

In general the span of the commutators is not very well behaved in ultraproducts, because the number of commutators needed may grow without bound. See [BF15]. 


\subsection{Hyponormality}

In $M_{n}$, many weaker notions of normality are equivalent to normality. One of these is hyponormality, originally due to Halmos:

Definition 12.4.1 ([Hal50], [Sta62]). $a$ is hyponormal if $a^{*} a \geq a a^{*}$.

Proposition 12.4.2. In finite-dimensional $\mathrm{C}^{*}$-algebras hyponormal implies normal.

Proof. If $a$ is hyponormal, then $a^{*} a-a a^{*}$ is positive and trace-zero so it is zero.

Example 12.4.3. Let $\mathcal{U}$ be a free ultrafilter on $\mathbb{N}$. Hyponormal elements in $\prod_{\mathcal{U}} M_{n}$ need not be normal. Consider

$$
a_{n}=\left[\begin{array}{ccccc}
0 & & & \\
1 & 0 & & & \\
& \sqrt{\frac{n-2}{n-1}} & 0 & & \\
& \ddots & \ddots & \\
& & \sqrt{\frac{1}{n-1}} & 0
\end{array}\right] \in M_{n} .
$$

Note $\left\|a_{n}\right\|=1$, and

$$
a_{n}^{*} a_{n}-a_{n} a_{n}^{*}=\operatorname{diag}\left(1,-\frac{1}{n-1}, \ldots,-\frac{1}{n-1}\right) \in M_{n}
$$

Note $\left\|a_{n}^{*} a_{n}-a_{n} a_{n}^{*}\right\|=1$, and if we let $x_{n}=\operatorname{diag}(1,0, \ldots, 0) \in M_{n}$, then $\|\left[a_{n}^{*}, a_{n}\right]-$ $x_{n} \|=1 /(n-1)$. Thus $\left(a_{n}^{*} a_{n}-a_{n} a_{n}^{*}\right)_{\mathcal{U}}=\left(x_{n}\right)$ is a non-zero projection, so it is positive. Thus $\left(a_{n}\right)_{\mathcal{U}}$ is hyponormal but not normal.

Another weakening of normality is subnormality, also due to Halmos: 
Definition 12.4.4 ([Hal50]). An operator $a \in \mathcal{B}(\mathcal{H})$ is subnormal if it has a normal extension. That is, there is a Hilbert space $\mathcal{K}$ containing $\mathcal{H}$ and a normal operator $n \in \mathcal{B}(\mathcal{K})$ so that

$$
n=\left(\begin{array}{ll}
a & b \\
0 & c
\end{array}\right)
$$

for some bounded operators $b: \mathcal{H}^{\perp} \rightarrow \mathcal{H}$ and $c: \mathcal{H}^{\perp} \rightarrow \mathcal{H}^{\perp}$.

In $M_{n}$ subnormal elements are normal, see [Hal82].

Question 12.4.5. Are subnormal elements of a pseudomatricial $\mathrm{C}^{*}$-algebra normal?

Embry's improvement of the Halmos-Bram criterion (see [Hal50], [Bra55], and [Emb73]) is a tempting approach, but we have the usual issues of bounding the number of terms needed to witness subnormality.

One can ask similar questions about other weaker forms of normality, such as quasinormal elements $\left(\left[a, a^{*} a\right]=0\right)$, and paranormal operators $\left(\left\|a^{2} \xi\right\| \geq\|a \xi\|^{2}\right.$ for all unit vectors $\xi)$.

\subsection{Projections and Traces}

Proposition 12.5.1. In pseudomatricial $\mathrm{C}^{*}$-algebras the projections are totally ordered by Murray-von Neumann subequivalence.

Proof. We show that "all projections are comparable" is an axiomatizable property. Certainly this property is preserved by $*$-isomorphism. Suppose the property holds 
in each $\mathfrak{A}_{i}$. Let $p$ and $q$ be projections in $\prod_{\mathcal{U}} \mathfrak{A}_{i}$, then without loss of generality there are representative sequences of projections $p=\left(p_{i}\right)_{\mathcal{U}}, q=\left(q_{i}\right)_{\mathcal{U}}$. Then

$$
I=\left\{i \mid p_{i} \prec q_{i}\right\} \sqcup\left\{i \mid p_{i} \text { is equivalent to } q_{i}\right\} \sqcup\left\{i \mid q_{i} \prec p_{i}\right\} \in \mathcal{U},
$$

where $\prec$ denotes Murray-von Neumann subequivalence. Thus exactly one of the disjoint sets above is in $\mathcal{U}$. Thus we have one of the following: (1) $p$ is subequivalent to $q$, (2) $p$ and $q$ are Murray-von Neumann equivalent, or (3) $q$ is subequivalent to $p$.

This property is preserved by ultraroots. Suppose $p$ and $q$ are projections in $\mathfrak{A}$, then $(p)_{\mathcal{U}}$ and $(q)_{\mathcal{U}}$ are projections in $\mathfrak{A}^{\mathcal{U}}$, so they are comparable. If they are Murray-von Neumann equivalent, then by 3.2.1 there are representative sequences of projections $(p)_{\mathcal{U}}=\left(p_{i}\right)_{\mathcal{U}}$ and $(q)_{\mathcal{U}}=\left(q_{i}\right)_{\mathcal{U}}$ and partial isometries $v_{i}$ so $v_{i}^{*} v_{i}=p_{i}$ and $v_{i} v_{i}^{*}=q_{i}$. Thus we have

$$
S=\left\{i:\left\|p-p_{i}\right\|<1 \text { and }\left\|q-q_{i}\right\|<1\right\} \in \mathcal{U}
$$

so for $i \in S, p$ is equivalent to $p_{i}$ which is equivalent to $q_{i}$ which is equivalent to $q$, so $p$ and $q$ are equivalent. The argument in the subequivalence case is similar.

In fact, this property is axiomatized by the following sentence:

$\sup _{p, q \text { projections } x} \inf _{x \text { partial isom }}\left(\left\|p-x^{*} x\right\|+\left\|\left(x x^{*}\right) q-x x^{*}\right\|\right) \cdot\left(\left\|q-x^{*} x\right\|+\left\|\left(x x^{*}\right) p-x x^{*}\right\|\right)=0$.

Corollary 12.5.2. If $\mathfrak{A}$ is a pseudomatricial $\mathrm{C}^{*}$-algebra, then $K_{0}(\mathfrak{A})$ is totally ordered. If $\mathfrak{A}$ is separable, then $K_{0}(\mathfrak{A})$ is a countable abelian totally ordered group, so it is a dimension group. See [EHS80]. 
Corollary 12.5.3. Let $\mathfrak{A}$ be an infinite-dimensional pseudomatricial $\mathrm{C}^{*}$-algebra. For every minimal projection $p, \tau(p)=0$. In particular, infinite-dimensional pseudomatrical algebras have a non-faithful tracial state, and they are not simple.

Proof. The identity $I$ dominates a minimal projection $m_{1}$. Then since $\mathfrak{A}$ is infinitedimensional, $I-m_{1}$ is non-zero and dominates another minimal projection $m_{2}$ orthogonal to $m_{1}$. Continuing in this way, for all $n \in \mathbb{N}$ we can iteratively find $n$ orthogonal minimal projections $m_{i}$. Since all minimal projections are Murray-von Neumann equivalent, so $\tau\left(m_{i}\right)=\tau\left(m_{j}\right)$ for all $i, j \in \mathbb{N}$. Thus

$$
1=\tau(I) \geq \tau\left(\sum_{i=1}^{n} m_{i}\right)=n \tau\left(m_{1}\right) .
$$

So for all $n$, the trace of a minimal projection is less than $1 / n$, so minimal projections must have trace zero.

Minimal projections in pseudomatrical $\mathrm{C}^{*}$-algebras behave like infinitesimal elements: they are non-zero and have norm one, but they are subequivalent to every projection with non-zero trace.

Note that the trace ideal $J$ of a separable, infinite-dimensional pseudomatricial $\mathrm{C}^{*}$-algebra $\mathfrak{A}$ contains a copy of the compacts (generated by any countable collection of orthogonal minimal projections - see 7.1.2 in [Bla98]), and the quotient $\mathfrak{A} / J$ has matrix units of all orders.

Proposition 12.5.4. Pseudomatrical $\mathrm{C}^{*}$-algebras have a unique tracial state. 
Proof. Since a pseudomatricial $\mathrm{C}^{*}$-algebra has real rank zero, the span of the projections is dense. Thus the trace is determined by its value on projections, and the trace on each projection is determined by how many orthogonal Murray-von Neumann equivalent copies of the projection (or approximate fractions of the projection) one can find.

More precisely, the maximum number of orthogonal Murray-von Neumann equivalent copies of $p$ is well-defined by the previous result and finiteness or cancellation. If there are $n$ orthogonal Murray-von Neumann equivalent copies of $p$, then $\tau(p) \leq 1 / n$. Now if $q$ is an approximate $d^{\text {th }}$ of $p$ then $\tau(q)=\tau(p) / d$. Repeating the process with smaller and smaller fractions of $p$ determines the trace of $p$ as precisely as we wish.

Proposition 12.5.5. Pseudomatrical algebras have the strong Dixmier property, i.e. for all $a \in \mathfrak{A}, \overline{\operatorname{conv}(\mathcal{U}(a))} \cap \mathcal{Z}(\mathfrak{A})$ is a singleton.

Proof. Note if $z \in \overline{\operatorname{conv}(\mathcal{U}(a))}$, then there are convex combinations

$$
\sum_{1}^{n} \lambda_{i} u_{i}^{*} a u_{i} \stackrel{\|\cdot\|}{\longrightarrow} z
$$

so that

$$
\tau(a)=\sum_{1}^{n} \lambda_{i} \tau(a)=\sum_{1}^{n} \lambda_{i} \tau\left(u_{i}^{*} a u_{i}\right)=\tau\left(\sum_{1}^{n} \lambda_{i} u_{i}^{*} a u_{i}\right) \rightarrow \tau(z)
$$

Thus if $z$ is central, $z=\lambda I$ and $\tau(z)=\lambda=\tau(a)$ we have $z=\tau(a) I$ is unique.

Corollary 12.5.6. If $J$ is a (closed, two-sided) ideal of a pseudomatricial $\mathrm{C}^{*}$-algebra $\mathfrak{A}$, and there is an $x \in J$ with $\tau(x) \neq 0$, then $J=\mathfrak{A}$. 
Proof. Since $x \in J$ is a closed, two-sided ideal, $\tau(x) I \in \overline{\operatorname{conv}(\mathcal{U}(x))} \|^{\|\cdot\|} \subseteq J$.

Proposition 12.5.7. Pseudomatricial $\mathrm{C}^{*}$-algebras have strict comparison of projections. That is, if $\tau(q)<\tau(p)$ then $q$ is equivalent to a proper subprojection of $p$.

Proof. Obviously this property holds in $M_{n}$ since the trace of a projection is the rank of the projection divided by $n$, and we have that $q$ is subequivalent to $p$ if and only $\operatorname{rank}(q) \leq \operatorname{rank}(p)$. Suppose each $\mathfrak{A}_{i}$ has strict comparison with respect to $\tau_{i}$. Then $\prod_{\mathcal{U}} \mathfrak{A}_{i}$ has strict comparison of projections with respect to $\tau=\lim _{\mathcal{U}} \tau_{i}$ : $\operatorname{Suppose}$ $p$ and $q$ are projections in $\prod_{\mathcal{U}} \mathfrak{A}_{i}$ with $\tau(p)<\tau(q)$. Without loss of generality, there are representative sequences of projections $p=\left(p_{i}\right)_{\mathcal{U}}, q=\left(q_{i}\right)_{\mathcal{U}}$. Since $\tau(p)=$ $\lim _{\mathcal{U}} \tau_{i}\left(p_{i}\right)<\lim _{\mathcal{U}} \tau_{i}\left(q_{i}\right)=\tau(q)$

$$
S=\left\{i \mid \tau_{i}\left(p_{i}\right)<\tau_{i}\left(q_{i}\right)\right\} \in \mathcal{U}
$$

Thus for $i \in S, p_{i}$ is properly Murray-von Neumann equivalent to a subprojection $q_{i}^{\prime}$ of $q_{i}$ via some partial isometry $v_{i}$. Considering the partial isometry $\left(v_{i}\right)_{\mathcal{U}}$, we see that $p$ is properly Murray-von Neumann subequivalent to $q$.

Suppose $\mathfrak{A}^{\mathcal{U}}$ has strict comparison of projections with respect to $\lim _{\mathcal{U}} \tau$ for some tracial state $\tau$ on $\mathfrak{A}$. Then $\mathfrak{A}$ has strict comparison of projection with respect to $\tau$ : Suppose $p, q$ are projections in $\mathfrak{A}$ with $\tau(p)<\tau(q)$. Then $P=(p)_{\mathcal{U}}$ and $Q=(q)_{\mathcal{U}}$ are projections in $\mathfrak{A}^{\mathcal{U}}$ with $\lim _{\mathcal{U}} \tau(p)_{\mathcal{U}}<\lim _{\mathcal{U}} \tau(q)_{\mathcal{U}}$. So by assumption, $P$ is properly Murray-von Neumann subequivalent to $Q$. So there is a partial isometry $V$ with $V^{*} V=P$ and $V V^{*}=Q^{\prime} \leq Q$. Without loss of generality, representative sequences 
of projections $q_{i}^{\prime} \leq q$ so $Q^{\prime}=\left(q_{i}^{\prime}\right)_{\mathcal{U}}$, and we can find partial isometries $v_{i}$ so $V=\left(v_{i}\right)_{\mathcal{U}}$ and on large set of indices, $v_{i}^{*} v_{i}=p$ and $v_{i} v_{i}^{*}=q_{i}^{\prime} \leq q$. So $p$ is Murray-von Neumann subequivalent to $q$.

The converse is not true because there are non-zero trace zero projections.

The same argument shows that pseudocompact $\mathrm{C}^{*}$-algebras have strict comparison of projections, that $q$ is Murray-von Neumann subequivalent to $p$ if for all traces $\tau$, $\tau(q)<\tau(p)$

In $M_{n}$, Murray-von Neumann equivalence, unitary equivalence, and homotopy equivalence of projections are all equivalent. The same property holds for pseudomatricial $\mathrm{C}^{*}$-algebras:

Proposition 12.5.8. In a pseudomatricial algebra, Murray-von Neumann equivalence, unitary equivalence, and homotopy equivalence are equivalent.

Proof. It is clear unitary equivalence implies Murray-von Neumann equivalence. We show that "Murray-von Neumann equivalence implies unitary equivalence" is an axiomatizable property. Of course this property is preserved by isomorphism. Suppose in $\mathfrak{A}_{i}$ Murray-von Neumann equivalence implies unitary equivalence. Let $p \sim q$ be Murray-von Neumann equivalent projections in $\prod_{\mathcal{U}} \mathfrak{A}_{i}$. Without loss of generality we have that $p=\left(p_{i}\right)_{\mathcal{U}}, q=\left(q_{i}\right)_{\mathcal{U}}$, with $p_{i}$ and $q_{i}$ projections, and a partial isometry $v=\left(v_{i}\right)_{\mathcal{U}}$ so that

$$
S=\left\{i \mid p_{i}=v_{i}^{*} v_{i} \text { and } q_{i}=v_{i} v_{i}^{*}\right\} \text { is in } \mathcal{U} \text {. }
$$


Thus for $i \in S, p_{i}$ and $q_{i}$ are unitarily equivalent in $\mathfrak{A}_{i}$, so there is a unitary $u_{i} \in \mathfrak{A}_{i}$ so that $u_{i}^{*} p_{i} u_{i}=q_{i}$. For $i \notin S$ let $u_{i}=I_{i}$. Letting $u=\left(u_{i}\right)_{\mathcal{U}}$ we have that $u$ is a unitary and $u^{*} p u=q$. Thus $p$ and $q$ are are unitarily equivalent in $\prod_{\mathcal{U}} \mathfrak{A}_{i}$.

Suppose Murray-von Neumann equivalence implies unitary equivalence in $\mathfrak{A}^{\mathcal{U}}$. Suppose $p$ and $q$ are Murray-von Neumann equivalent projections in $\mathfrak{A}$ via a partial isometry $v$, then $(p)_{\mathcal{U}}$ and $(q)_{\mathcal{U}}$ are Murray-von Neumann equivalent in $\mathfrak{A}^{\mathcal{U}}$ via $(v)_{\mathcal{U}}$. Then there is a unitary $u \in \mathfrak{A}^{\mathcal{U}}$ so $u^{*}(p)_{\mathcal{U}} u=(q)_{\mathcal{U}}$. Without loss of generality $u=\left(u_{i}\right)_{\mathcal{U}}$ where the $u_{i}$ are unitaries in $\mathfrak{A}$ and $\left(u_{i} p u_{i}^{*}\right)_{\mathcal{U}}=(q)_{\mathcal{U}}$, then

$$
S=\left\{i:\left\|u_{i} p u_{i}^{*}-q\right\|<1\right\} \text { is in } \mathcal{U}
$$

If two projections are distance less that one apart, they are unitarily equivalent (See e.g. 2.2.4 and 2.2.6 in [Bla98]). Thus for $i \in S, u_{i} p u_{i}^{*}$ is unitarily equivalent to $q$. So $p$ and $q$ are unitarily equivalent.

It is clear that homotopy equivalence implies Murray-von Neumann equivalence. Since exponential unitaries are dense in the unitaries of a pseudomatricial $\mathrm{C}^{*}$-algebra, unitary equivalence via a unitary in the connected component of the identity, $\mathcal{U}_{0}(\mathfrak{A})$ implies homotopy equivalence, we see that unitary equivalence is equivalent to homotopy equivalence in pseudocompact $\mathrm{C}^{*}$-algebras. 


\section{6 $K_{0}$ Groups}

Notice that if $\mathfrak{A}$ is pseudomatricial, then for any natural number $n, M_{n}(\mathfrak{A})$ is pseudomatricial. The induced trace on $M_{n}(\mathfrak{A})$ from $\mathfrak{A}$ is just $n$ times the unique tracial state on $M_{n}(\mathfrak{A})$. Elements of $K_{0}(\mathfrak{A})$ are of the form $[p]_{0}-[q]_{0}$ where $p, q$ are projections in some $M_{n}(\mathfrak{A})$, so either $p \preceq q$ or vice-versa. In the first case, $p$ is the orthogonal sum of a projection $p^{\prime}$ and a projection $q^{\prime}$ which is Murray-von Neumann equivalent to $q$. Thus $[p]_{0}-[q]_{0}=\left[p^{\prime}+q^{\prime}\right]_{0}-[q]_{0}=\left[p^{\prime}\right]_{0}+\left[q^{\prime}\right]_{0}-[q]_{0}=\left[p^{\prime}\right]_{0}$. So $K_{0}(\mathfrak{A})=\left\{ \pm[p]_{0} \mid p \in M_{n}(\mathfrak{A})\right\}$.

The K-theory of ultraproducts has been studied in [Li05]: the $K_{0}$ group is a sort of graded ultraproduct of the $K_{0}$ groups of the components, since a projection in $K_{0}\left(\prod_{\mathcal{U}} \mathfrak{A}_{i}\right)$ is in some $M_{n}\left(\prod_{\mathcal{U}} \mathfrak{A}_{i}\right)=\prod_{\mathcal{U}} M_{n}\left(A_{i}\right)$. In other words, the matrix amplifications need to be bounded.

Let $G$ be a (totally) ordered additive group, $g, h \in G$. We let $|g|$ denote the element of $\{g,-g\}$ which is greater than or equal to the zero element. Recall $g$ is Archimedean less than $h$, denoted $g \ll h$ if $n|g| \leq|h|$ for all natural numbers $n$. We say $g, h \in G$ are Archimedean equivalent, denoted $g \approx h$, if there are natural numbers $n, m$ so that $n|g| \geq|h|$ and $m|h| \geq|g|$.

Proposition 12.6.1. Let $\mathfrak{A}$ be a pseudomatricial $\mathrm{C}^{*}$-algebra. Then $\operatorname{ker}\left(K_{0}(\tau)\right)$ is the subgroup of $K_{0}(\mathfrak{A})$ generated by the trace-zero projections, and it is a subgroup of $\mathbb{R}^{\eta}$, real-valued functions from well-ordered subsets of $\eta$, the set of Archimedean 
equivalence classes of trace zero projections, equipped with the lexicographical ordering.

Proof. It is clear that $\operatorname{ker}\left(K_{0}(\tau)\right)=\left\{ \pm[p]_{0} \mid \tau(p)=0\right\}$ is a subgroup of $K_{0}$. If $m$ is a (non-zero) minimal projection then $g-[m]_{0}$ and $g+[m]_{0}$ are the greatest element less than $g$ and the least element greater than $g$ respectively. Since $\operatorname{ker}\left(K_{0}(\tau)\right)$ is a totally ordered abelian group, by the Hahn Embedding Theorem [Hah07], $\operatorname{ker}\left(K_{0}(\tau)\right)$ is a subgroup of $\mathbb{R}^{\eta}$, the group of functions from well-ordered subsets of $\eta$ into $\mathbb{R}$, where $\eta$ is the set of Archimedian equivalence classes of trace zero projections, equipped with the lexicographical ordering. Note that if $\mathfrak{A}$ is separable, these groups are countable, so $\eta$ is countable.

Proposition 12.6.2. Let $\mathfrak{A}$ be a pseudomatricial $\mathrm{C}^{*}$-algebra. Then $K_{0}(\mathfrak{A}) \cong G \oplus$ $\operatorname{ker}\left(K_{0}(\tau)\right)$ as ordered abelian groups, where $G$ is a divisible subgroup of $\mathbb{R}$, equipped with the usual lexicographical order.

Proof. (Sketch:) Let $\mathfrak{A}$ be a pseudomatricial $\mathrm{C}^{*}$-algebra, then

$$
K_{0}(\mathfrak{A}) \cong K_{0}(\mathfrak{A}) / \operatorname{ker}\left(K_{0}(\tau)\right) \oplus \operatorname{ker}\left(K_{0}(\tau)\right)
$$

We just need to show that $K_{0}(\mathfrak{A}) / \operatorname{ker}\left(K_{0}(\tau)\right)$ is a subgroup of $\mathbb{R}$. This group is isomorphic to the image of $K_{0}(\tau)$, which is just all rational multiples of trace on projections in $\mathfrak{A}$. If $\lambda$ is the trace of a projection $p$ in $\mathfrak{A}$ then $n \lambda$ is the trace of $\operatorname{diag}(p, \ldots, p) \in M_{n}(\mathfrak{A})$, and also $\lambda / n$ is the trace of an approximate splitting of $p$ into $n$ Murray-von Neumann equivalent pieces. 
Proposition 12.6.3. Let $G$ be a countable divisible subgroup of $\mathbb{R}$ and $S$ be a countable subset of $[0,1]$. We can find a separable pseudomatricial $\mathrm{C}^{*}$-algebra $\mathfrak{A}$ so that $K_{0}(\mathfrak{A}) \supseteq G \oplus\left(\mathbb{Z}^{S}\right)$ as ordered abelian groups when $G \oplus\left(\mathbb{Z}^{S}\right)$ is given the usual lexicographical ordering.

Proof. Consider $\mathfrak{A}=\prod_{\mathcal{U}} M_{n}$ where $\mathcal{U}$ is a free ultrafilter on $\mathbb{N}$. For $s \in S$, let $p_{n}^{(s)}$ be a rank $\left\lfloor n^{s}\right\rfloor$ projection in $M_{n}$. Consider $P_{s}=\left(p_{n}^{(s)}\right)_{\mathcal{U}}$, then $\left\{P_{s}\right\}_{s \in S}$ is a countable family of projections in $\mathfrak{A}$. Note that

$$
\tau\left(P_{s}\right)=\lim _{\mathcal{U}} \tau_{n}\left(p_{n}^{(s)}\right)=\lim _{\mathcal{U}} \frac{\left\lfloor n^{s}\right\rfloor}{n}=0
$$

so these are trace zero projections. Also, in $K_{0}(\mathfrak{A}),\left[P_{s}\right]_{0} \gg\left[P_{r}\right]_{0}$ when $s>r$ are in $S$.

Similarly, for $\lambda \in G \cap[0,1]$ for all $n$ we can take $q_{n}$ to be a rank $\lfloor n \lambda\rfloor$ projection in $M_{n}$. Then consider $Q_{\lambda}=\left(q_{n}\right)_{\mathcal{U}} \in \mathfrak{A}$.

$$
\tau\left(Q_{\lambda}\right)=\lim _{\mathcal{U}} \tau_{n}\left(q_{n}\right)_{\mathcal{U}}=\lim _{\mathcal{U}} \frac{\lfloor n \lambda\rfloor}{n}=\lambda
$$

We can apply the downward Löwenheim-Skolem theorem to the countable set $\left\{P_{s}, Q_{\lambda}\right\}_{s \in S, \lambda \in G \cap[0,1]}$ to get a separable elementary $\mathrm{C}^{*}$-subalgebra $\mathfrak{A}_{0}$ of $\mathfrak{A}$ which contains all the $P_{r}$ and $Q_{m}$. In particular, this is a pseudomatrical $\mathrm{C}^{*}$-algebra, so the restriction of the trace on $\mathfrak{A}$ is the trace on $\mathfrak{A}_{0}$. Also, for $n \in \mathbb{N}, r<s$ in $S$, the sentences $\phi_{n, r, s}$ with parameters from $\mathfrak{A}_{0}$ which say "there are $n$ orthogonal projections Murray-von Neumann equivalent to $P_{r}$ whose sum is dominated by $P_{s}$ " hold in $\mathfrak{A}_{0}$ 
for all $n$ and $r$. $\left[P_{r}\right]_{0} \ll\left[P_{s}\right]_{0}$ in $K_{0}\left(\mathfrak{A}_{0}\right)$ as well. Thus we have shown the range of the trace on projections contains all of $G \cap[0,1]$.

\subsection{Other Finiteness Properties}

Interestingly, pseudomatricial $\mathrm{C}^{*}$-algebras seem to fail to have many nice finiteness properties, such as nuclearity, exactness, and quasidiagonality.

Proposition 12.7.1 (7.2.4 in $\left.\left[\mathrm{FHL}^{+} 16\right]\right)$. An infinite-dimensional pseudomatricial $\mathrm{C}^{*}$-algebra is never nuclear.

The idea of the proof is that the associated von Neumann algebra does not have property $\Gamma$ so it is not hyperfinite.

It is possible for pseudocompact $\mathrm{C}^{*}$-algebras to be nuclear, but only in a trivial way:

Example 12.7.2. Let $\mathcal{U}$ be a free ultrafilter on $\mathbb{N}$. Then $\prod_{i \rightarrow \mathcal{U}} M_{n}^{\oplus i} \cong M_{n}\left(\prod_{\mathcal{U}} \mathbb{C}^{i}\right)$ is an infinite-dimensional, $n$-subhomogeneous pseudocompact $\mathrm{C}^{*}$-algebra, and thus nuclear.

The class of $n$-subhomogenous $\mathrm{C}^{*}$-algebras are axiomatizable by the AmitsurLevizki theorem, see [AL50] and 2.5.d in $\left[\mathrm{FHL}^{+} 16\right]$.

Proposition 12.7.3 (3.12.1 in $\left.\left[\mathrm{FHL}^{+} 16\right]\right)$. For a $\mathrm{C}^{*}$-algebra $\mathfrak{A}$, the following are equivalent: 
1. Every $\mathrm{C}^{*}$-algebra elementarily equivalent to $\mathfrak{A}$ is nuclear.

2. Every $\mathrm{C}^{*}$-algebra elementarily equivalent to $\mathfrak{A}$ is exact.

3. $\mathfrak{A}$ is subhomogeneous.

Question 12.7.4. Can infinite-dimensional pseudomatricial $\mathrm{C}^{*}$-algebras be exact? Can they be quasidiagonal? 


\section{Chapter 13}

\section{Pseudocompact Embeddable}

\section{Algebras}

\subsection{MF Algebras}

Definition 13.1.1. A separable $\mathrm{C}^{*}$-algebra is an $M F$ algebra (for matricial field or M. Fell) if it can be written as an inductive limit of a generalized inductive system of finite dimensional $\mathrm{C}^{*}$-algebras.

A good reference is [BK97], see also [Fel61] and [Lee77].

Theorem 13.1.2 (3.2.2 in [BK97], 11.1.5 in [BO08]). Let $\mathfrak{A}$ be a separable $\mathrm{C}^{*}$ algebra. The following are equivalent:

1. $\mathfrak{A}$ is an MF algebra. 
2. $\mathfrak{A}$ is isomorphic to an inductive limit of a generalized inductive system of finitedimensional $\mathrm{C}^{*}$-algebras

3. $\mathfrak{A}$ can be embedded as a $\mathrm{C}^{*}$-subalgebra of $\left(\prod_{n \in \mathbb{N}} M_{k_{n}}\right) /\left(\bigoplus_{n \in \mathbb{N}} M_{k_{n}}\right)$ for some sequence of natural numbers $\left(k_{n}\right)_{n \in \mathbb{N}}$.

4. $\mathfrak{A}$ has an essential QD extension by $\mathcal{K}(\mathcal{H})$.

5. $\mathfrak{A}$ is a limit of a continuous field of matrix algebras.

6. $\mathfrak{A}$ admits norm microstates: for all $d \in \mathbb{N}, x_{1}, \ldots, x_{d}$ self-adjoint elements, $\mathfrak{P}$ a finite set of noncommutative polynomials in $d$ variables, $\varepsilon>0$, there is an $n \in \mathbb{N}$ and $X_{1}, \ldots, X_{d} \in M_{n}(\mathbb{C})$ so that for each $P \in \mathfrak{P}$,

$$
\left|\left\|P\left(x_{1}, \ldots, x_{d}\right)\right\|-\left\|P\left(X_{1}, \ldots, X_{d}\right)\right\|\right|<\varepsilon .
$$

Proposition 13.1.3 (3.3.1 - 8 in [BK97]).

1. Subalgebras of an MF algebra are MF.

2. Every RFD algebra is an MF algebra.

3. Every separable $\mathrm{C}^{*}$-algebra is a quotient of an MF algebra.

4. MF algebras are stably finite.

Proposition 13.1.4 (3.3.5 in [BK97]). If $\mathfrak{A}$ is an MF algebra, then $\mathfrak{A}$ can be embed$\operatorname{ded}$ in $\left(\prod_{n \in \mathbb{N}} \mathfrak{F}_{n}\right) /\left(\bigoplus_{n \in \mathbb{N}} \mathfrak{F}_{n}\right)$ where each $\mathfrak{F}_{n}$ is finite-dimensional, and for all $x \in \mathfrak{A}$, 
the image of $a,\left(x_{n}\right) \in\left(\prod \mathfrak{F}_{n}\right) /\left(\bigoplus \mathfrak{F}_{n}\right)$ satisfies

$$
\lim _{n \rightarrow \infty}\left\|x_{n}\right\|=\|x\|
$$

Corollary 13.1.5. If $\mathfrak{A}$ is an MF algebra, then $\mathfrak{A}$ can be embedded (not necessarily unitally) into a pseudocompact $\mathrm{C}^{*}$-algebra.

Proof. We have that $\mathfrak{A}$ can be embedded in $\left(\prod_{n \in \mathbb{N}} \mathfrak{F}_{n}\right) /\left(\bigoplus_{n \in \mathbb{N}} \mathfrak{F}_{n}\right)$ where each $\mathfrak{F}_{n}$ is finite-dimensional. Let $\mathcal{U}$ be a free ultrafilter on $\mathbb{N}$. Let $\pi$ is the quotient map

$$
\pi:\left(\prod_{n \in \mathbb{N}} \mathfrak{F}_{n}\right) /\left(\bigoplus_{n \in \mathbb{N}} \mathfrak{F}_{n}\right) \rightarrow \prod_{\mathcal{U}} \mathfrak{F}_{n}
$$

which sends

$$
\left(x_{n}\right) \mapsto\left(x_{n}\right)_{\mathcal{U}}
$$

Then we have that $\left.\pi\right|_{\mathfrak{A}}$ is injective, so $\mathfrak{A}$ is $*$-isomorphic to its image in $\prod_{\mathcal{U}} \mathfrak{F}_{n}$.

In fact, the converse is true. Recall the following result:

Theorem 13.1.6 (4.1 in [GH01]). Let $\mathfrak{A}$ and $\mathfrak{B}$ be $\mathrm{C}^{*}$-algebras. The following are equivalent:

1. $\mathfrak{A}$ is $*$-isomorphic to a subalgebra of an ultrapower of $\mathfrak{B}$.

2. For any $x_{1}, \ldots, x_{d}$ in $\mathfrak{A}, *$-polynomials $p_{1}, \ldots, p_{d}$ in $d$ variables, and positive numbers $r_{1}, s_{1}, \ldots, r_{n}, s_{n}$ such that $r_{k}<\left\|p_{k}\left(x_{1}, \ldots, x_{d}\right)\right\|<s_{k}$ for $1 \leq k \leq n$, there are $y_{1}, \ldots, y_{d} \in \mathfrak{B}$ such that $r_{k}<\left\|p_{k}\left(y_{1}, \ldots, y_{d}\right)\right\|<s_{k}$ for $1 \leq k \leq n$. 
3. There is a net of maps $\left(\phi_{\lambda}\right)_{\lambda \in \Lambda}$ whose domains form an increasing directed collection of subsets of $\mathfrak{A}$ whose union $\mathcal{D}$ is dense in $\mathfrak{A}$, whose ranges are contained in $\mathfrak{B}$, and for all $x, y \in \mathcal{D}$ and $r, s \in \mathbb{Q}+i \mathbb{Q}$, we have that

(a) The $\phi_{\lambda}$ are asymptotically linear:

$$
\lim _{\lambda}\left\|\phi_{\lambda}\left(r x+s y^{*}\right)-\left(r \phi_{\lambda}(x)+s \phi_{\lambda}(y)^{*}\right)\right\|=0
$$

(b) The $\phi_{\lambda}$ are asymptotically multiplicative:

$$
\lim _{\lambda}\left\|\phi_{\lambda}(x y)-\phi_{\lambda}(x) \phi_{\lambda}(y)\right\|=0
$$

(c) The $\phi_{\lambda}$ are asymptotically injective: $\liminf \operatorname{in}_{\lambda}\left\|\phi_{\lambda}(x)\right\| \geq\|x\| / 2$

Using these ideas and the norm microstate criterion for being MF, we get the following result, which is known:

Corollary 13.1.7. A separable $C^{*}$-algebra $\mathfrak{A}$ is a subalgebra of pseudocompact $C^{*}$ algebras if and only if it is a $\mathrm{MF} \mathrm{C}^{*}$-algebra.

Proof. Suppose $\mathfrak{A}$ is a subalgebra of a pseudocompact $\mathrm{C}^{*}$-algebra $\prod_{\mathcal{U}} F_{i}$ where the $F_{i}$ are finite-dimensional, so up to isomorphism $F_{i} \subseteq M_{d_{i}}$ for some $d_{i}$. We show that $\mathfrak{A}$ admits norm microstates. Let $d \in \mathbb{N}, x_{1}, \ldots, x_{d} \in \mathfrak{A}, \mathfrak{P}$ a set of non-commutative polynomials, and $\varepsilon>0$ be given. Since $\mathfrak{A} \subseteq \prod_{\mathcal{U}} F_{i}$, take representative sequences $x_{j}=\left(y_{j}^{(i)}\right)_{i \rightarrow \mathcal{U}}$ for $1 \leq j \leq d$. Then for each $p \in \mathfrak{F}$,

$$
\left\|p\left(x_{1}, \ldots, x_{d}\right)\right\|=\lim _{\mathcal{U}}\left\|p\left(y_{1}^{(i)}, \ldots, y_{d}^{(i)}\right)\right\|
$$


So the set

$$
S_{p}=\left\{i:\|\| p\left(x_{1}, \ldots, x_{d}\right)\|-\| p\left(y_{1}^{(i)}, \ldots, y_{d}^{(i)}\right) \| \mid<\varepsilon\right\} \text { is in } \mathcal{U}
$$

Let $i_{0} \in \bigcap_{p \in \mathfrak{P}} S_{p} \in \mathcal{U}$. Then let $X_{j}=y_{j}^{\left(i_{0}\right)} \in F_{i} \subseteq M_{d_{i}}$, these give the desired norm microstates.

Conversely, if $\mathfrak{A}$ is sepearble and admits norm microstates, let $\left(x_{j}\right)_{j \in \mathbb{N}}$ be dense in the self-adjoint elements of $\mathfrak{A}$. Define the index set $I$ by

$$
I=\{(d, \mathfrak{P}, \varepsilon): d \in \mathbb{N}, \mathfrak{P} \text { a finite set of polynomials in } d \text {-variables, } \varepsilon>0\}
$$

Then $I$ is a partially ordered set under $(d, \mathfrak{P}, \varepsilon) \prec\left(d^{\prime}, \mathfrak{P}^{\prime}, \varepsilon^{\prime}\right)$ if and only if $d \leq d^{\prime}$, $\mathfrak{P} \subseteq \mathfrak{P}^{\prime}$ (ignoring extra variables), and $\varepsilon \geq \varepsilon^{\prime}$. Then the collection of upward directed sets form a filter. Take an ultrafilter $\mathcal{U}$ dominating this order filter.

For each $i=(d, \mathfrak{P}, \varepsilon) \in I$, there are matrices $X_{1}^{(i)}, \ldots, X_{d}^{(i)}$ in $M_{d_{i}}$ satisfying the norm-microstate definition for $x_{1}, \ldots, x_{d}$. We define a map $\Phi$ from $\bigcup_{j \in \mathbb{N}} \operatorname{span}\left(x_{j}\right)$ to $\prod_{\mathcal{U}} M_{d_{i}}$ as follows: If $x \in \bigcup_{j \in \mathbb{N}} \operatorname{span}\left(x_{j}\right)$, then $x=\sum_{j=1}^{d} \lambda_{j} x_{j}$ (we take the minimum d). We define $\Phi(x)=\left(y_{i}\right)_{\mathcal{U}}$, where

$$
y_{i}=\sum_{j=1}^{d} \lambda_{j} X_{j}^{(i)} \in M_{d_{i}} .
$$

as long as $i=\left(d^{\prime}, \mathfrak{P}, \varepsilon\right)$ has $d^{\prime} \geq d$. Then this map is asymptotically linear, multiplicative, $*$-preserving, and norm-preserving. To see this, let $\varepsilon_{0}>0$ be given, then for those $i=(d, \mathfrak{P}, \varepsilon) \in I$ with $\varepsilon<\varepsilon_{0}, \mathfrak{P}$ containing the appropriate polynomials, the desired results will hold up to $\varepsilon_{0}$. Since $\bigcup_{j \in \mathbb{N}} \operatorname{span}\left(x_{j}\right)$ is dense in $\mathfrak{A}, \Phi$ extends to a $*$-embedding of $\mathfrak{A}$ into $\prod_{\mathcal{U}} M_{d_{i}}$. 
This type of construction of approximately embedding finite-dimensional subspaces is very common, see Proposition 6.2 and Theorem 6.3 in [Hei80]. These ideas can be stated in terms of existential theories, see Theorem 1.7 in [Hen76].

\subsection{Embeddings}

Proposition 13.2.1. Every pseudocompact $\mathrm{C}^{*}$-algebra is a unital subalgebra of a pseudomatricial $\mathrm{C}^{*}$-algebra.

Proof. If $\mathfrak{F}$ is finite-dimensional, there is a $d$ and $n_{1}, \ldots n_{d} \in \mathbb{N}$ so that

$$
\mathfrak{F} \cong \bigoplus_{i=1}^{d} M_{n_{i}}
$$

See e.g. 7.1 .5 in [RLL00]. So $\mathfrak{F}$ embeds unitally into $M_{n}$ where $n=\sum_{i=1}^{d} n_{i}$. If $\mathfrak{A}$ is pseudocompact, then there ultrafilters $\mathcal{U}$ and $\mathcal{V}$ and finite-dimensional $\mathrm{C}^{*}$-algebras $\mathfrak{F}_{i}$ which embed unitally into some $M_{d_{i}}$ so that

$$
\mathfrak{A} \hookrightarrow \mathfrak{A}^{\mathcal{V}} \cong\left(\prod_{\mathcal{U}} \mathfrak{F}_{i}\right)^{\mathcal{V}} \hookrightarrow\left(\prod_{\mathcal{U}} M_{d_{i}}\right)^{\mathcal{V}} .
$$

Thus $\mathfrak{A}$ is a unital subalgebra of a pseudomatricial $\mathrm{C}^{*}$-algebra.

Since an infinite-dimensional pseudomatricial $\mathrm{C}^{*}$-algebra $\mathfrak{A}$ has $d$ Murray-von Neumann equivalent mutually orthogonal projections, we can embed $M_{d}$ (not necessarily unitally) into $\mathfrak{A}$. Thus

Corollary 13.2.2. An infinite-dimensional pseudomatrical algebra contains every finite-dimensional $\mathrm{C}^{*}$-algebra (but not necessarily unitally). 
The following example shows that the unitization of the compact operators on a separable Hilbert space embeds unitally into any infinite-dimensional ultraproduct of matrix algebras. This again shows that subalgebras of pseudomatricial $\mathrm{C}^{*}$-algebras need not be pseudomatricial.

Example 13.2.3. Let $\mathcal{H}$ be a separable, countably infinite-dimensional Hilbert space. Pick an orthonormal basis $\left\{\xi_{n}\right\}_{n \in \mathbb{N}}$ of $\mathcal{H}$ and let $\mathcal{H}_{n}=\operatorname{span}\left(\xi_{1}, \ldots, \xi_{n}\right)$. Let $p_{n}$ be the projection onto $\mathcal{H}_{n}$. Then $p_{n}$ are increasing and converge in the strong operator to the identity. Let $\mathcal{U}$ be a free ultrafilter on $\mathbb{N}$. Then there is a map $\Phi$ from $\mathcal{K}(\mathcal{H})$ into $\prod_{\mathcal{U}} \mathcal{B}\left(\mathcal{H}_{n}\right) \cong \prod_{\mathcal{U}} M_{n}$, which sends an operator $x$ to $\left(p_{n} x p_{n}\right)_{\mathcal{U}}$

This map is linear, *-preserving, and isometric:

$$
\begin{gathered}
\Phi(x+\lambda y)=\left(p_{n}(x+\lambda y) p_{n}\right)_{\mathcal{U}}=\left(p_{n} x p_{n}\right)_{\mathcal{U}}+\lambda\left(p_{i} y p_{i}\right)_{\mathcal{U}}=\Phi(x)+\lambda \Phi(y) \\
\Phi\left(x^{*}\right)=\left(p_{n} x^{*} p_{n}\right)_{\mathcal{U}}=\left(p_{n} x p_{n}\right)_{\mathcal{U}}^{*}=\Phi(x)^{*}
\end{gathered}
$$

To see that $\Phi$ is isometric, let $\varepsilon>0$ and take a vector $\xi$ so $\|x \xi\|>\|x\|-\varepsilon / 4$, then since $p_{n}$ converge strongly to the identity, for $n$ sufficiently large $\left\|\xi-p_{i} \xi\right\|<\varepsilon / 4\|x\|$ and $\left\|p_{n} x \xi-x \xi\right\|<\varepsilon / 4$. Then

$$
\begin{aligned}
\left\|p_{n} x p_{n} \xi-x \xi\right\| & \leq\left\|p_{n} x p_{n} \xi-p_{n} x \xi\right\|+\left\|p_{n} x \xi-x \xi\right\|+\left\|x \xi-x p_{n} \xi\right\| \\
& <\left\|p_{n} x\right\| \cdot\left\|p_{n} \xi-\xi\right\|+\frac{\varepsilon}{4}+\|x\| \cdot\left\|\xi-p_{n} \xi\right\|<\frac{3}{4 \varepsilon} .
\end{aligned}
$$

Thus

$$
\left\|p_{n} x p_{n} \xi\right\| \geq\|x \xi\|-\left\|p_{n} x p_{n} \xi-x \xi\right\|>\|x\|-\varepsilon
$$


We see that

$$
\|\Phi(x)\|=\lim _{\mathcal{U}}\left\|p_{n} x p_{n}\right\| \geq\|x\|-\varepsilon
$$

However, $\Phi$ is not multiplicative if we expand the domain to $\mathcal{B}(\mathcal{H})$. One can see this by considering $s$ the shift on $\mathcal{B}(\mathcal{H})$ sending $\xi_{n}$ to $\xi_{n+1}$ and seeing that $p_{n} s^{*} p_{n} s p_{n}$ is distance 1 from $p_{n} s^{*} s p_{n}=p_{n}$ for all $n \in \mathbb{N}$, as the former kills $\xi_{n}$ and the latter preserves it.

However, this map is multiplicative on $\mathcal{K}(\mathcal{H})$. It suffices to consider finite rank operators since $\Phi$ is bounded, and from the above we only need to show that $\Phi$ is multiplicative on finite rank operators $f_{1}$ and $f_{2}$. Let $\varepsilon>0$ be given. Let $\hat{\mathcal{H}}_{0}=$ $\operatorname{span}\left(f_{1} \mathcal{H}, \operatorname{coker}\left(f_{1}\right), f_{2} \mathcal{H}, \operatorname{coker}\left(f_{2}\right)\right)$. Since $p_{n}$ converge strongly to the identity, we can find an $n_{0}$ so that $\left\|p_{n} \xi-\xi\right\|<\varepsilon\|\xi\|$ for all $\xi \in \hat{\mathcal{H}}_{0}$. Then for all $n \geq n_{0}$ we have that

$$
\begin{aligned}
\left\|\Phi\left(f_{1}\right) \Phi\left(f_{2}\right)-\Phi\left(f_{1} f_{2}\right)\right\| & =\left\|p_{n} f_{1} p_{n} f_{2} p_{n}-p_{n} f_{1} f_{2} p_{n}\right\| \\
& =\sup _{\xi \in \mathfrak{F}_{n}}\left\|p_{n} f_{1} p_{n} f_{2} p_{n} \xi-p_{n} f_{1} f_{2} p_{n} \xi\right\| \\
& \leq\left\|p_{n}\right\|\left\|f_{1}\right\|\left\|p_{n} f_{2} \xi-f_{2} \xi\right\|<\varepsilon .
\end{aligned}
$$

In particular, we have that $\mathcal{K}(\mathcal{H})$ and its unitization $\mathcal{K}(\mathcal{H})^{\sim}$ is $*$-isomorphic to a $\mathrm{C}^{*}$-subalgebra of $\prod_{\mathcal{U}} \mathfrak{F}_{n}$. This map is not surjective.

In fact, the finite-dimensional subalgebras of $\prod_{\mathcal{U}} \mathfrak{A}_{i}$ are close to finite-dimensional subspaces of the $\mathfrak{A}_{i}$. This is not surprising in light of section 6 of [Hei80]. 
Proposition 13.2.4. If $\mathfrak{F}$ is a finite-dimensional subalgebra of $\prod_{\mathcal{U}} \mathfrak{A}_{i}$, then there is an $S \in \mathcal{U}$ so for all $i \in S, \mathfrak{A}_{i}$ contains a subalgebra $*$-isomorphic to $\mathfrak{F}$.

Proof. Let $\varepsilon>0$ and $\mathfrak{F}$ be a finite dimensional subalgebra of $\prod_{\mathcal{U}} \mathfrak{A}_{n}$, take matrix units for $\mathfrak{F}$. Take representative sequences. The equations defining matrix units are weakly stable (see e.g. 3.2.5.(8),(9) in $\left[\mathrm{FHL}^{+} 16\right], 4.9$ in [Lor93], 2.25-2.28 in [Bla85]), so on a large subset of indices, the components are within $\delta$ of satisfying the matrix unit equations. So they are within distance $\varepsilon$ of matrix units in those components. See also III.3.2 in [Dav96].

Question 13.2.5. Can we classify the pseudocompacts as direct sums or direct integrals of pseudomatrical over their center?

See 4.1 in [FHS13] for a similar argument in the tracial case.

If $\mathfrak{A}$ is separable and pseudocompact, then $\mathcal{Z}(\mathfrak{A})$ is separable and pseudocompact, so it is some $\mathcal{C}(X)$ with $X$ compact, Hausdorff, with a countable dense subset of isolated points. If $x_{0} \in X$ is an isolated point, the characteristic function $p=\chi_{x_{0}} \in$ $\mathcal{C}(X)$ is a minimal central projection. Then $p_{0} \mathfrak{A} p_{0}$ is pseudocompact and has no nontrivial central projections, so it is pseudomatricial. 


\section{Chapter 14}

\section{Summary and Open Questions}

In conclusion, we have shown that pseudocompact $\mathrm{C}^{*}$-algebras are unital, tracial, real rank zero, stable rank one, and have trivial $K_{1}$ groups, and Dixmier's property. We showed that the pseudocompact $\mathrm{C}^{*}$-algebras are stable under direct sums, and both pseudocompact and pseudomatricial algebras are stable under tensoring by $M_{n}$ for any $n \in \mathbb{N}$, but in general subalgebras of pseudocompact $\mathrm{C}^{*}$-algebras are not pseudocompact. Corners and centers of pseudocompact $\mathrm{C}^{*}$-algebras are pseudocompact. We axiomatized the class of commutative pseudocompact $\mathrm{C}^{*}$-algebras. We showed in a pseudocompact $\mathrm{C}^{*}$-algebra that every non-zero projection dominates a minimal projection, and a non-zero projection $p$ is minimal if and only if $p \mathfrak{A} p=\mathbb{C} p$. We showed that a pseudocompact $\mathrm{C}^{*}$-algebra is pseudomatricial if and only if it has trivial center. In a pseudomatricial $\mathrm{C}^{*}$-algebra, for any $d$ the identity can be written as a sum of $d$ orthogonal Murray-von Neumann equivalent projections plus fewer than $d$ 
orthogonal minimal projections. We showed that pseudomatricial $\mathrm{C}^{*}$-algebras have a unique tracial trace, strict comparison of projections, and their $K_{0}$ groups are totally ordered with successors and predecessors. The unique trace on an infinite-dimensional pseudomatricial $\mathrm{C}^{*}$-algebra is not faithful, so they are not simple. We showed that the class of pseudocompact $\mathrm{C}^{*}$-algebras are distinct from the UHF and AF algebras as well as compact operators on a Hilbert space.

We conclude with a list of open questions about the pseudocompact and pseudomatricial $\mathrm{C}^{*}$-algebras.

Question 14.0.6. Is the trace on a pseudomatricial algebra amenable? (This is a necessary condition for being quasidiagonal)

Question 14.0.7. Is the trace ideal $\left\{x \mid \tau\left(x^{*} x\right)=0\right\}=\{x \mid$ for all $y, \tau(y x)=0\}$ the ideal generated by the trace-zero projections?

Question 14.0.8. Can the trace ideal be isomorphic to the compacts? $\operatorname{Can} \operatorname{ker}\left(K_{0}(\tau)\right) \cong$ $\mathbb{Z}$ ? Is it possible for the range of the trace on projections be $[0,1] \cap \mathbb{Q}$ ?

Question 14.0.9. Is the trace ideal maximal? What is the quotient of a pseudomatricial $\mathrm{C}^{*}$-algebra by the trace ideal?

Question 14.0.10. Do the ideals in a pseudocompact $\mathrm{C}^{*}$-algebra correspond to order ideals in $K_{0}(\mathfrak{A})$ ? Are these the same as the Archimedean equivalence classes?

Question 14.0.11. Can we find an explicit example of a separable, infinite-dimensional pseudomatricial $\mathrm{C}^{*}$-algebra? 
We probably should not expect to, since ultraproducts are non-constructible.

Question 14.0.12. Can we find an explicit axiomatization of the pseudocompact and pseudomatricial $\mathrm{C}^{*}$-algebras?

Question 14.0.13. Are pseudomatricial $\mathrm{C}^{*}$-algebras ever exact? Are pseudomatricial $\mathrm{C}^{*}$-algebras ever quasidiagonal?

The list of properties of pseudocompact $\mathrm{C}^{*}$-algebras is remarkably similar to the properties of the $\mathrm{C}^{*}$-algebras studied in [Phi04] and [PV13] except those algebras are simple. It would be interesting to know if infinite-dimensional pseudocompact $\mathrm{C}^{*}$ algebras are isomorphic, or even elementarily equivalent to their opposite algebras. See also [GS15a].

Question 14.0.14. Are pseudocompact $C^{*}$-algebras closed under (say minimal) tensor products?

This is unlikely, generally tensor products do not play well with continuous logic. See Section 3.8 in $\left[\mathrm{FHL}^{+} 16\right]$. 


\section{Bibliography}

[AFHV84] C. Apostol, L. A. Fialkow, Domingo A. Herrero, and D. Voiculescu. Approximation of Hilbert space operators. Vol. II, volume 102 of Research Notes in Mathematics. Pitman (Advanced Publishing Program), Boston, MA, 1984.

[AL50] A. S. Amitsur and J. Levitzki. Minimal identities for algebras. Proc. Amer. Math. Soc., 1:449-463, 1950.

[Ale27] P. Alexandroff. Über stetige Abbildungen kompakter Räume. Math. Ann., 96(1):555-571, 1927.

[AM57] A. A. Albert and B. Muckenhoupt. On matrices of trace zero. Michigan Math. J., 4:1-3, 1957.

[Arc78] R. J. Archbold. On the norm of an inner derivation of a $C^{*}$-algebra. Math. Proc. Cambridge Philos. Soc., 84(2):273-291, 1978. 
[AW04] C. Akemann and N. Weaver. Consistency of a counterexample to Naimark's problem. Proc. Natl. Acad. Sci. USA, 101(20):7522-7525, 2004 .

[Ax68] J. Ax. The elementary theory of finite fields. Ann. of Math. (2), 88:239$271,1968$.

[Ban23] S. Banach. Sur le probleme de la mesure. Fund. Math., 4:8, 1923.

[BF15] T. Bice and I. Farah. Traces, Ultrapowers and the Pedersen-Petersen C*-Algebras. Houston J. Math., 41(4):1175-1190, 2015.

[BK97] B. Blackadar and E. Kirchberg. Generalized inductive limits of finitedimensional $C^{*}$-algebras. Math. Ann., 307(3):343-380, 1997.

[Bla78] B. Blackadar. Weak expectations and nuclear $C^{*}$-algebras. Indiana Univ. Math. J., 27(6):1021-1026, 1978.

[Bla85] B. Blackadar. Shape theory for $C^{*}$-algebras. Math. Scand., 56(2):249$275,1985$.

[Bla98] B. Blackadar. K-theory for operator algebras, volume 5 of Mathematical Sciences Research Institute Publications. Cambridge University Press, Cambridge, second edition, 1998.

[Bla06] B. Blackadar. Operator algebras, volume 122 of Encyclopaedia of Mathematical Sciences. Springer-Verlag, Berlin, 2006. Theory of $C^{*}$-algebras 
and von Neumann algebras, Operator Algebras and Non-commutative Geometry, III.

[BN09] S. Baratella and S.-A. Ng. Nonstandard Hulls of C*-Algebras. ArXiv e-prints, October 2009.

[BN11] S. Baratella and S.-A. Ng. Some properties of nonstandard hulls of Banach algebras. Bull. Belg. Math. Soc. Simon Stevin, 18(1):31-38, 2011.

[BO08] N. P. Brown and N. Ozawa. $C^{*}$-algebras and finite-dimensional approximations, volume 88 of Graduate Studies in Mathematics. American Mathematical Society, Providence, RI, 2008.

[BP91] L. G. Brown and G. K. Pedersen. $C^{*}$-algebras of real rank zero. $J$. Funct. Anal., 99(1):131-149, 1991.

[Bra55] J. Bram. Subnormal operators. Duke Math. J., 22:75-94, 1955.

[Bra72] O. Bratteli. Inductive limits of finite dimensional $C^{*}$-algebras. Trans. Amer. Math. Soc., 171:195-234, 1972.

[BT79] M. W. Boyd and W. R. Transue. Properties of ultraproducts. Rend. Circ. Mat. Palermo (2), 28(3):387-397 (1980), 1979.

[BY03] I. Ben-Yaacov. Positive model theory and compact abstract theories. $J$. Math. Log., 3(1):85-118, 2003. 
[BYBHU08] I. Ben Yaacov, A. Berenstein, C. W. Henson, and A. Usvyatsov. Model theory for metric structures. In Model theory with applications to algebra and analysis. Vol. 2, volume 350 of London Math. Soc. Lecture Note Ser., pages 315-427. Cambridge Univ. Press, Cambridge, 2008.

$\left[\mathrm{CCF}^{+} 14\right] \quad$ K. Carlson, E. Cheung, I. Farah, A. Gerhardt-Bourke, B. Hart, L. Mezuman, N. Sequeira, and A. Sherman. Omitting types and AF algebras. Arch. Math. Logic, 53(1-2):157-169, 2014.

[Cho88] M. D. Choi. Almost commuting matrices need not be nearly commuting. Proc. Amer. Math. Soc., 102(3):529-533, 1988.

[CK66] C. C. Chang and H. J. Keisler. Continuous model theory. Annals of Mathematics Studies, No. 58. Princeton Univ. Press, Princeton, N.J., 1966.

[CM58] C. C. Chang and A. C. Morel. On closure under direct product. J. Symb. Logic, 23:149-154, 1958.

[CM79] J. B. Conway and B. B. Morrel. Operators that are points of spectral continuity. Integral Equations Operator Theory, 2(2):174-198, 1979.

[CM81] J. B. Conway and B. B. Morrel. Operators that are points of spectral continuity. II. Integral Equations Operator Theory, 4(4):459-503, 1981. 
[CM83] J. B. Conway and B. B. Morrel. Operators that are points of spectral continuity. III. Integral Equations Operator Theory, 6(3):319-344, 1983.

[CMS06] Barbara F. Csima, Antonio Montalbán, and Richard A. Shore. Boolean algebras, Tarski invariants, and index sets. Notre Dame J. Formal Logic, 47(1):1-23, 2006.

[CN74] W. W. Comfort and S. Negrepontis. The theory of ultrafilters. SpringerVerlag, New York-Heidelberg, 1974. Die Grundlehren der mathematischen Wissenschaften, Band 211.

[Com77] W. W. Comfort. Ultrafilters: some old and some new results. Bull. Amer. Math. Soc., 83(4):417-455, 1977.

[Com80] W. W. Comfort. Ultrafilters: an interim report. In Surveys in general topology, pages 33-54. Academic Press, New York-London-Toronto, Ont., 1980.

[Con91] J. B. Conway. The theory of subnormal operators, volume 36 of Mathematical Surveys and Monographs. American Mathematical Society, Providence, RI, 1991.

[Dav96] K. R. Davidson. $C^{*}$-algebras by example, volume 6 of Fields Institute Monographs. American Mathematical Society, Providence, RI, 1996. 
[Daw08] M. Daws. Ultrapowers of Banach algebras and modules. Glasg. Math. J., 50(3):539-555, 2008.

[Daw09] M. Daws. Amenability of ultrapowers of Banach algebras. Proc. Edinb. Math. Soc. (2), 52(2):307-338, 2009.

[Daw10] M. Daws. Corrigendum: Amenability of ultrapowers of Banach algebras. Proc. Edinb. Math. Soc. (2), 53(3):633-637, 2010.

[DCK72] D. Dacunha-Castelle and J. L. Krivine. Applications des ultraproduits à l'étude des espaces et des algèbres de Banach. Studia Math., 41:315-334, 1972.

[Dix96] J. Dixmier. Les algèbres d'opérateurs dans l'espace hilbertien (algèbres de von Neumann). Les Grands Classiques Gauthier-Villars. [GauthierVillars Great Classics]. Éditions Jacques Gabay, Paris, 1996. Reprint of the second (1969) edition.

$\left[\mathrm{EFH}^{+} 14\right] \quad$ C. J. Eagle, I. Farah, B. Hart, B. Kadets, V. Kalashnyk, and M. Lupini. Fraïssé limits of C*-algebras. ArXiv e-prints, November 2014.

[EFKV15] C. J. Eagle, I. Farah, E. Kirchberg, and A. Vignati. Quantifier elimination in $\mathrm{C}^{*}$-algebras. ArXiv e-prints, February 2015.

[EHS80] E. G. Effros, D. E. Handelman, and C.-L. Shen. Dimension groups and their affine representations. Amer. J. Math., 102(2):385-407, 1980. 
[EL89] R. Exel and T. Loring. Almost commuting unitary matrices. Proc. Amer. Math. Soc., 106(4):913-915, 1989.

[Ell76] G. A. Elliott. On the classification of inductive limits of sequences of semisimple finite-dimensional algebras. J. Algebra, 38(1):29-44, 1976.

[Emb73] M. R. Embry. A generalization of the Halmos-Bram criterion for subnormality. Acta Sci. Math. (Szeged), 35:61-64, 1973.

[Erš64] J. L. Eršov. Decidability of the elementary theory of relatively complemented lattices and of the theory of filters. Algebra $i$ Logika Sem., $3(3): 17-38,1964$.

[EV14] C. J. Eagle and A. Vignati. Saturation of $C^{*}$-algebras. ArXiv e-prints, June 2014.

[EV15] C. J. Eagle and A. Vignati. Saturation and elementary equivalence of $C^{*}$-algebras. J. Funct. Anal., 269(8):2631-2664, 2015.

[Far11] I. Farah. All automorphisms of the Calkin algebra are inner. Ann. of Math. (2), 173(2):619-661, 2011.

[Far14] I. Farah. Logic and operator algebras. ArXiv e-prints, April 2014.

[Fel61] J. M. G. Fell. The structure of algebras of operator fields. Acta Math., 106:233-280, 1961. 
[FGHS16] I. Farah, I. Goldbring, B. Hart, and D. Sherman. Existentially closed $\mathrm{II}_{1}$ factors. 233(2):173-196, 2016.

[FH11] J. Fang and D. Hadwin. A note on the invariant subspace problem relative to a type $\mathrm{II}_{1}$ factor. Houston J. Math., 37(3):879-893, 2011.

$\left[\mathrm{FHL}^{+} 16\right] \quad$ I. Farah, B. Hart, M. Lupini, L. Robert, A. Tikuisis, A. Vignati, and W. Winter. The Model Theory of Nuclear $\mathrm{C}^{*}$-algebras. ArXiv e-prints, February 2016.

[FHS13] I. Farah, B. Hart, and D. Sherman. Model theory of operator algebras I: stability. Bull. Lond. Math. Soc., 45(4):825-838, 2013.

[FHS14a] I. Farah, B. Hart, and D. Sherman. Model theory of operator algebras II: model theory. Israel J. Math., 201(1):477-505, 2014.

[FHS14b] I. Farah, B. Hart, and D. Sherman. Model theory of operator algebras III: elementary equivalence and $\mathrm{II}_{1}$ factors. Bull. Lond. Math. Soc., 46(3):609-628, 2014.

[FM14] I. Farah and M. Magidor. Omitting types in logic of metric structures. ArXiv e-prints, November 2014.

[FMS58] T. Frayne, A. C. Morel, and D. S. Scott. Set-theoretical properties of reduced products. Amer. Math. Soc. Notices, 5:674, 1958. 
[FMS63a] T. Frayne, A. C. Morel, and D. S. Scott. Reduced direct products. Fund. Math., 51:195-228, 1962/1963.

[FMS63b] T. Frayne, A. C. Morel, and D. S. Scott. Correction to the paper "Reduced direct products". Fund. Math., 53:117, 1963.

[FMS13] I. Farah, P. McKenney, and E. Schimmerling. Some Calkin algebras have outer automorphisms. Arch. Math. Logic, 52(5-6):517-524, 2013.

[FR96] P. Friis and M. Rørdam. Almost commuting self-adjoint matrices - a short proof of Huaxin Lin's theorem. J. Reine Angew. Math., 479:121$131,1996$.

[FS58] T. Frayne and D. S. Scott. Model-theoretical properties of reduced products. Amer. Math. Soc. Notices, 5:675, 1958.

[FS10] I. Farah and S. Shelah. A dichotomy for the number of ultrapowers. $J$. Math. Log., 10(1-2):45-81, 2010.

[FST58] T. Frayne, D. S. Scott, and A. Tarski. Reduced products. Amer. Math. Soc. Notices, 5:673-674, 1958.

[FTT13] I. Farah, A. Toms, and A. Törnquist. The descriptive set theory of $\mathrm{C}^{*}-$ algebra invariants. Int. Math. Res. Not. IMRN, (22):5196-5226, 2013. 
[GH01] L. Ge and D. Hadwin. Ultraproducts of $C^{*}$-algebras. In Recent advances in operator theory and related topics (Szeged, 1999), volume 127 of Oper. Theory Adv. Appl., pages 305-326. Birkhäuser, Basel, 2001.

[Gha15] Saeed Ghasemi. $S A W^{*}$-algebras are essentially non-factorizable. Glasg. Math. J., 57(1):1-5, 2015.

[GL15] I. Goldbring and V. Lopes. Pseudofinite and Pseudocompact Metric Structures. Notre Dame J. Form. Log., 56(3):493-510, 2015.

[Gli60] J. G. Glimm. On a certain class of operator algebras. Trans. Amer. Math. Soc., 95:318-340, 1960.

[Gol15] I. Goldbring. Model theory and the QWEP conjecture. ArXiv e-prints, November 2015.

[GS15a] I. Goldbring and T. Sinclair. Games and elementary equivalence of $\mathrm{II}_{1^{-}}$ factors. Pacific J. Math., 278(1):103-118, 2015.

[GS15b] I. Goldbring and T. Sinclair. On Kirchberg's embedding problem. J. Funct. Anal., 269(1):155-198, 2015.

[Hah07] H. Hahn. Über die nichtarchimedischen größensysteme. 116:601655, 1907. (in German).

[Hal50] P. R. Halmos. Normal dilations and extensions of operators. Summa Brasil. Math., 2:125-134, 1950. 
[Hal82] P. R. Halmos. A Hilbert space problem book, volume 19 of Graduate Texts in Mathematics. Springer-Verlag, New York-Berlin, second edition, 1982. Encyclopedia of Mathematics and its Applications, 17.

[Hal77] P. R. Halmos. Some unsolved problems of unknown depth about operators on Hilbert space. Proc. Roy. Soc. Edinburgh Sect. A, 76(1):67-76, $1976 / 77$.

[Hau14] F. Hausdorff. Grundzüge der mengenlehre. Math. Ann., 75:428-472, 1914.

[Hau27] F. Hausdorff. Mengenlehre, zweite, neubearbeitete Auflage. Verlag Walter de Gruyter \& Co., Berlin, 1927.

[Hei80] S. Heinrich. Ultraproducts in Banach space theory. J. Reine Angew. Math., 313:72-104, 1980.

[Hen75] C. W. Henson. When do two Banach spaces have isometrically isomorphic nonstandard hulls? Israel J. Math., 22(1):57-67, 1975.

[Hen76] C. W. Henson. Nonstandard hulls of Banach spaces. Israel J. Math., 25(1-2):108-144, 1976.

[Hew48] E. Hewitt. Rings of real-valued continuous functions. I. Trans. Amer. Math. Soc., 64:45-99, 1948. 
[HM74] C. W. Henson and L. C. Moore, Jr. Nonstandard hulls of the classical Banach spaces. Duke Math. J., 41:277-284, 1974.

[HRR07] C. W. Henson, Y. Raynaud, and A. Rizzo. On axiomatizability of noncommutative $L_{p}$-spaces. Canad. Math. Bull., 50(4):519-534, 2007.

[HS86] Domingo A. Herrero and Stanisław J. Szarek. How well can an $n \times n$ matrix be approximated by reducible ones? Duke Math. J., 53(1):233248, 1986.

[Jan72] G. Janssen. Restricted ultraproducts of finite von Neumann algebras. In Contributions to non-standard analysis (Sympos., Oberwolfach, 1970), pages 101-114. Studies in Logic and Found. Math., Vol. 69. NorthHolland, Amsterdam, 1972.

[JOS13] William B. Johnson, Narutaka Ozawa, and Gideon Schechtman. A quantitative version of the commutator theorem for zero trace matrices. Proc. Natl. Acad. Sci. USA, 110(48):19251-19255, 2013.

[Kad57] R. V. Kadison. Irreducible operator algebras. Proc. Nat. Acad. Sci. U.S.A., 43:273-276, 1957.

[KBMR12] A. Kanel-Belov, S. Malev, and L. Rowen. The images of noncommutative polynomials evaluated on $2 \times 2$ matrices. Proc. Amer. Math. Soc., 140(2):465-478, 2012. 
[KBMR16] A. Kanel-Belov, S. Malev, and L. Rowen. The images of multilinear polynomials evaluated on $3 \times 3$ matrices. Proc. Amer. Math. Soc., 144(1):7$19,2016$.

[KLR67] R. V. Kadison, E. C. Lance, and J. R. Ringrose. Derivations and automorphisms of operator algebras. II. J. Functional Analysis, 1:204-221, 1967.

[Lee77] R. Y. Lee. On $C^{*}$-algebras which are approachable by finite-dimensional $C^{*}$-algebras. Bull. Inst. Math. Acad. Sinica, 5(2):265-283, 1977.

[Li05] W. Li. On ultraproducts of operator algebras. Sci. China Ser. A, 48(9):1284-1295, 2005.

[Lin97] H. Lin. Almost commuting selfadjoint matrices and applications. In Operator algebras and their applications (Waterloo, ON, 1994/1995), volume 13 of Fields Inst. Commun., pages 193-233. Amer. Math. Soc., Providence, RI, 1997.

[Lor93] Terry A. Loring. $C^{*}$-algebras generated by stable relations. J. Funct. Anal., 112(1):159-203, 1993.

[Lor97] T. A. Loring. Lifting solutions to perturbing problems in $C^{*}$-algebras, volume 8 of Fields Institute Monographs. American Mathematical Society, Providence, RI, 1997. 
[Łoś55] J. Łoś. Quelques remarques, théorèmes et problèmes sur les classes définissables d'algèbres. In Mathematical interpretation of formal systems, pages 98-113. North-Holland Publishing Co., Amsterdam, 1955.

[LT70] W. A. J. Luxemburg and R. F. Taylor. Almost commuting matrices are near commuting matrices. Nederl. Akad. Wetensch. Proc. Ser. A 73=Indag. Math., 32:96-98, 1970.

[Lux69] W. A. J. Luxemburg. A general theory of monads. In Applications of Model Theory to Algebra, Analysis, and Probability (Inte rnat. Sympos., Pasadena, Calif., 1967), pages 18-86. Holt, Rinehart and Winston, New York, 1969.

[McD70] D. McDuff. Central sequences and the hyperfinite factor. Proc. London Math. Soc. (3), 21:443-461, 1970.

[Moo76] L. C. Moore, Jr. Hyperfinite extensions of bounded operators on a separable Hilbert space. Trans. Amer. Math. Soc., 218:285-295, 1976.

[Moo81] L. C. Moore, Jr. Approximately finite-dimensional Banach spaces. J. Funct. Anal., 42(1):1-11, 1981.

[New51] J. D. Newburgh. The variation of spectra. Duke Math. J., 18:165-176, 1951. 
[OHP13] A. Ould Houcine and F. Point. Alternatives for pseudofinite groups. J. Group Theory, 16(4):461-495, 2013.

[Oza13] N. Ozawa. About the Connes embedding conjecture: algebraic approaches. Jpn. J. Math., 8(1):147-183, 2013.

[Phi04] N. C. Phillips. A simple separable $C^{*}$-algebra not isomorphic to its opposite algebra. Proc. Amer. Math. Soc., 132(10):2997-3005 (electronic), 2004.

[PV13] N. C. Phillips and M. G. Viola. A simple separable exact C*-algebra not anti-isomorphic to itself. Math. Ann., 355(2):783-799, 2013.

[PW07] N. C. Phillips and N. Weaver. The Calkin algebra has outer automorphisms. Duke Math. J., 139(1):185-202, 2007.

[Rie08] F. Riesz. Stetigkeitsbegriff und abstrakte mengenlehre. Atti del IV Congress Internaz. Mat. Roma, 2:18-24, 1908.

[Rie83] M. A. Rieffel. Dimension and stable rank in the $K$-theory of $C^{*}$-algebras. Proc. London Math. Soc. (3), 46(2):301-333, 1983.

[Rin78] J. R. Ringrose. Derivations of quotients of von Neumann algebras. Proc. London Math. Soc. (3), 36(1):1-26, 1978. 
[RLL00] M. Rørdam, F. Larsen, and N. Laustsen. An introduction to K-theory for $C^{*}$-algebras, volume 49 of London Mathematical Society Student Texts. Cambridge University Press, Cambridge, 2000.

[Rør88] M. Rørdam. Advances in the theory of unitary rank and regular approximation. Ann. of Math. (2), 128(1):153-172, 1988.

[Ros69] P. Rosenthal. Research Problems: Are Almost Commuting Matrices Near Commuting Matrices? Amer. Math. Monthly, 76(8):925-926, 1969.

[Rud57] W. Rudin. Continuous functions on compact spaces without perfect subsets. Proc. Amer. Math. Soc., 8:39-42, 1957.

[RZ60] A. Robinson and E. Zakon. Elementary properties of ordered abelian groups. Trans. Amer. Math. Soc., 96:222-236, 1960.

[Sak62] S. Saki. The theory of $\mathrm{W}^{*}$-algebras, 1962.

[She09] D. Sherman. Notes on automorphisms of ultrapowers of $\mathrm{II}_{1}$ factors. Studia Math., 195(3):201-217, 2009.

[Sho37] K. Shoda. Einige Sätze über Matrizen. Jpn. J. Math., 13(3):361-365, 1937.

[Sim82] B. Sims. "Ultra"-techniques in Banach space theory, volume 60 of Queen's Papers in Pure and Applied Mathematics. Queen's University, Kingston, ON, 1982. 
[Sko34] T. Skolem. Über die Nicht-charakterisierbarkeit der Zahlenreihe mittels endlich oder abzählbar unendlich vieler Aussagen mit ausschliesslich Zahlenvariablen. Fund. Math., 23:150-161, 1934.

[Sta62] Joseph G. Stampfli. Hyponormal operators. Pacific J. Math., 12:14531458, 1962.

[Tar30] A. Tarski. Une contribution à la théorie de la mesure. volume 15, pages 42-50. 1930.

[Tar49] A. Tarski. Arithmetical classes and types of boolean algebras. Fund. Math., 55:64, 1949.

[TE05] L. N. Trefethen and M. Embree. Spectra and pseudospectra. Princeton University Press, Princeton, NJ, 2005. The behavior of nonnormal matrices and operators.

[Tho58] R. C. Thompson. On matrix commutators. J. Washington Acad. Sci., 48:306-307, 1958.

[Tre92] L. N. Trefethen. Pseudospectra of matrices. In Numerical analysis 1991 (Dundee, 1991), volume 260 of Pitman Res. Notes Math. Ser., pages 234-266. Longman Sci. Tech., Harlow, 1992.

[Tre97] L. N. Trefethen. Pseudospectra of linear operators. SIAM Rev., 39(3):383-406, 1997. 
[TT94] K.-C. Toh and L. N. Trefethen. Pseudozeros of polynomials and pseudospectra of companion matrices. Numer. Math., 68(3):403-425, 1994.

[Ula29] S. Ulam. Concerning functions of sets. Fund. Math., 14:231-233, 1929.

[Vää03] J. Väänänen. Pseudo-finite model theory. Mat. Contemp., 24:169183, 2003. 8th Workshop on Logic, Language, Informations and Computation-WoLLIC'2001 (Brasília).

[vN42] John von Neumann. Approximative properties of matrices of high finite order. Portugaliae Math., 3:1-62, 1942.

[Voi76] Dan Voiculescu. A non-commutative Weyl-von Neumann theorem. Rev. Roumaine Math. Pures Appl., 21(1):97-113, 1976.

[Voi83] D. Voiculescu. Asymptotically commuting finite rank unitary operators without commuting approximants. Acta Sci. Math. (Szeged), 45(14):429-431, 1983.

[Wea07] N. Weaver. Set theory and $C^{*}$-algebras. Bull. Symbolic Logic, 13(1):120, 2007.

[Wil70] J. P. Williams. Finite operators. Proc. Amer. Math. Soc., 26:129-136, 1970.

[Wil95] J. S. Wilson. On simple pseudofinite groups. J. London Math. Soc. (2), 51(3):471-490, 1995. 
[Wri54] F. B. Wright. A reduction for algebras of finite type. Ann. of Math. (2), $60: 560-570,1954$.

[Zsi73] L. Zsidó. The norm of a derivation in a $W^{*}$-algebra. Proc. Amer. Math. Soc., 38:147-150, 1973. 